\title{
Metal Halide Hydrates as Lewis Acid Catalysts for the Conjugated Friedel-Crafts Reactions of Indoles and Activated Olefins
}

\author{
Cristiane S. Schwalm, Marco Antonio Ceschi and Dennis Russowsky* \\ Instituto de Química, Universidade Federal do Rio Grande do Sul, Av. Bento Gonçalves 9500, \\ 91501-970 Porto Alegre-RS, Brazil
}

\begin{abstract}
$\mathrm{O}$ uso de haletos metálicos hidratados, tais como, $\mathrm{SnCl}_{2} \cdot 2 \mathrm{H}_{2} \mathrm{O}, \mathrm{MnCl}_{2} \cdot 4 \mathrm{H}_{2} \mathrm{O}, \mathrm{SrCl}_{2} \cdot 6 \mathrm{H}_{2} \mathrm{O}$, $\mathrm{CrCl}_{2} \cdot 6 \mathrm{H}_{2} \mathrm{O}, \mathrm{CoCl}_{2} \cdot 6 \mathrm{H}_{2} \mathrm{O}$ e $\mathrm{CeCl}_{3} \cdot 7 \mathrm{H}_{2} \mathrm{O}$ como catalisadores ácidos de Lewis foi investigado nas reações conjugadas tipo Friedel-Crafts entre indóis e olefinas ativadas. A utilização de enonas alifáticas forneceu as respectivas $\beta$-indolilcetonas à temperatura ambiente em longos tempos de reação, enquanto que nas reações com chalconas foi necessário o uso de condições de refluxo em isopropanol. As reações dos indóis com nitroestirenos foram efetuadas tanto em fase líquida como em condições sem solvente. Em todos os casos estudados foram obtidos rendimentos de razoáveis a bons.
\end{abstract}

Metal halide hydrates such as $\mathrm{SnCl}_{2} \cdot 2 \mathrm{H}_{2} \mathrm{O}, \mathrm{MnCl}_{2} \cdot 4 \mathrm{H}_{2} \mathrm{O}, \mathrm{SrCl}_{2} \cdot 6 \mathrm{H}_{2} \mathrm{O}, \mathrm{CrCl}_{2} \cdot 6 \mathrm{H}_{2} \mathrm{O}, \mathrm{CoCl}_{2} \cdot 6 \mathrm{H}_{2} \mathrm{O}$ e $\mathrm{CeCl}_{3} \cdot 7 \mathrm{H}_{2} \mathrm{O}$ were investigated as mild Lewis acids catalysts for the conjugate Friedel-Crafts reaction between indoles and activated olefins. The reactions were carried out with aliphatic unsaturated ketones over a period of days at room temperature, while chalcones reacted only under reflux conditions. The reactions with nitrostyrenes were either performed in solvent or under solventless conditions. In all cases reasonable to good yields were obtained.

Keywords: Friedel-Crafts, indoles, activated olefins, metal halide hydrates, Lewis acids, Michael addition

\section{Introduction}

The investigation of synthetic methodologies for the preparation of 3-substituted indole derivatives has been a continuous challenge in the chemistry of heterocyclic compounds. ${ }^{1-3}$

The $\mathrm{C}_{3}$ alkylation reaction of indoles through the conjugated Friedel-Crafts (CFC) reaction has received much attention in the last decade. ${ }^{4-6}$ Not only they allow access to advanced building blocks in the synthesis of natural compounds, but also their diverse biological properties of derivatives have pharmaceutical applications..$^{7-11}$

Over the past few years a range of methods for the preparation of $\beta$-indolylketones by CFC reaction of indoles with $\alpha, \beta$-unsaturated ketones has appeared in the literature in which both Brønsted or Lewis acidic catalysts have successfully been employed. However, in the curse of Brønsted acid catalysis, many procedures involve strong acidic conditions, expensive reagents, longer reaction times,

*e-mail: dennis@iq.ufrgs.br low yields, complex handling procedures and problematic side reactions, such as dimerization and polymerization. ${ }^{12}$

On the other hand, the employment of suitable metal halides such as $\mathrm{InCl}_{3},{ }^{13} \mathrm{InBr}_{3},{ }^{14} \mathrm{SmI}_{3},{ }^{15} \mathrm{GaI}_{3},{ }^{16} \mathrm{SbCl}_{3},{ }^{17}$ $\mathrm{ScCl}_{3},{ }^{18} \mathrm{AlCl}_{3},{ }^{19} \mathrm{ZrCl}_{4},{ }^{20}$ and $\mathrm{HfCl}_{4}{ }^{18}$ as Lewis acid catalysts have attracted attention due to their ability to efficiently activate the $\alpha, \beta$-unsaturated carbonyl system for the CFC reaction. However, many of them are moisture sensitive needing a careful handling and the achievement of a substitute is welcome. Additionally, others environmentally-benign, oxygen and/or moisture tolerant Lewis acids such as $\mathrm{Yb}(\mathrm{OTf})_{3},{ }^{7} \mathrm{Sc}(\mathrm{OTf})_{3},{ }^{21} \mathrm{Bi}(\mathrm{OTf})_{3},{ }^{22}$ $\mathrm{Hf}(\mathrm{OTf})_{4},{ }^{21}$ and $\mathrm{Zr}(\mathrm{OTf})_{4}{ }^{23}$ have been applied to this transformation, but they are expensive.

A few previous uses of metal salt hydrates such as: $\mathrm{NaAuCl}_{4} \cdot 2 \mathrm{H}_{2} \mathrm{O},{ }^{24} \mathrm{ZrOCl}_{2} \cdot 8 \mathrm{H}_{2} \mathrm{O},{ }^{25} \mathrm{RuCl}_{3} \cdot \mathrm{nH}_{2} \mathrm{O}^{26}$ and $\mathrm{Fe}\left(\mathrm{BF}_{4}\right)_{2} \cdot 6 \mathrm{H}_{2} \mathrm{O}^{27}$ have been reported.

As a part of our ongoing investigation searching for moisture compatible Lewis acids, including their use in protic solvents, for the synthesis of small molecules of biological interest, ${ }^{28-36}$ we decide to investigate the 
metal halide hydrates as a potential catalysts for the CFC reaction. We have earlier demonstrated the successful use of $\mathrm{SnCl}_{2} \cdot 2 \mathrm{H}_{2} \mathrm{O}$ as an alcoholic compatible Lewis acid catalyst for the multicomponent dihydropyrimidinone synthesis. ${ }^{37}$ The Lewis acidity of $\mathrm{SnCl}_{2} \cdot 2 \mathrm{H}_{2} \mathrm{O}$ has been reported by others in different reactions types. ${ }^{38-40}$

Although the hydrogen Brønsted acid catalysis has been suggested by Spencer as the main process when a metal halide is used as a Lewis catalyst in aqueous (or protic alcoholic) solvents, ${ }^{41}$ other authors postulated the water-tolerance of many metal halides. Kobayashi suggests that the certain metal halides are able to complex with the electrophile before the hydrolysis occurs. These characteristics depends on the hydrolysis constant $\left(\mathrm{K}_{\mathrm{h}}\right)$ within a certain range and a high water-exchange rate constant (WERC).${ }^{42}$ On the other hand, Fringuelli et al. ${ }^{43}$ report the use of $\mathrm{AlCl}_{3}, \mathrm{TiCl}_{4}$, and $\mathrm{SnCl}_{4}$ as the Lewis acids catalysts in aqueous solvent. Although the authors agree with the fast hydrolysis of metal salts, they affirm that the hydrated metal cation remains as the effective species as Lewis acid catalyst.

Recent studies of Lindstrom ${ }^{44}$ show that the water tightly coordinated to the metal halide become more labile upon complexation of an organic ligand (electrophile), leading to a higher rate of exchange with substrate molecules and consequently to higher reaction rates.

The $\mathrm{SnCl}_{2} \cdot 2 \mathrm{H}_{2} \mathrm{O}$ was employed as a Lewis acid catalyst in the esterification of oleic acid. The authors proved the ethanol-compatibility of the catalyst as a Lewis acid through the recovery of $\mathrm{SnCl}_{2} \cdot 2 \mathrm{H}_{2} \mathrm{O}$ and verified that the catalytic activity of the recovered $\mathrm{SnCl}_{2} \cdot 2 \mathrm{H}_{2} \mathrm{O}$ remains unaltered in successive reactions with oleic acid. ${ }^{45}$

Based on these previous studies, we report in this paper, our efforts to apply a series of metal halide hydrates such as: $\mathrm{SnCl}_{2} \cdot 2 \mathrm{H}_{2} \mathrm{O}, \mathrm{MnCl}_{2} \cdot 4 \mathrm{H}_{2} \mathrm{O}, \mathrm{SrCl}_{2} \cdot 6 \mathrm{H}_{2} \mathrm{O}, \mathrm{CoCl}_{2} \cdot 6 \mathrm{H}_{2} \mathrm{O}$ and $\mathrm{CrCl}_{3} \cdot 6 \mathrm{H}_{2} \mathrm{O}$ as Lewis acid catalysts for the CFC reaction of indoles and activated olefins, carried out in benign alcoholic solvents.

\section{Results and Discussion}

\section{Reactions of indoles with alkylidene-alkylketones}

In our first experiment, we investigated the use of various metal halide hydrates as Lewis acid catalysts in the reaction of indole (1a) and 4-hexen-3-one (2a) under
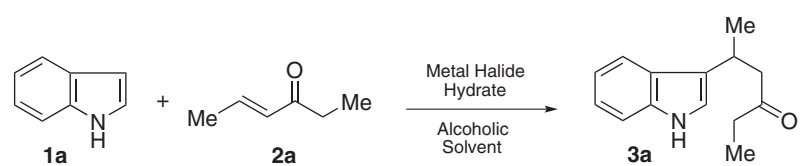

Scheme 1. Synthesis of $\beta$-indolylketone $\mathbf{3 a}$. different alcoholic solvents, temperatures and catalyst loading. In all experiments the desired $\beta$-indolylketone 3a was obtained in good yield (Scheme 1).

As shown in Table 1, the yield of the product 3a was quite good when the reaction was carried out in $i-\mathrm{PrOH}$ at room temperature. The reaction proceeded cleanly ${ }^{46}$ as observed by comparative TLC and $i$-PrOH was chosen as the default solvent for the other reactions (c.f. entries 1-3).

Table 1. Synthesis of $\beta$-indolylketone 3a under different conditions

\begin{tabular}{lcccccc}
\hline entry & $\begin{array}{c}\text { Lewis Acid } \\
\text { (equiv.) }\end{array}$ & Solvent & Temperature & $\begin{array}{c}\text { time } \\
(\mathrm{h})\end{array}$ & $\begin{array}{c}\text { Yield } \\
(\%)\end{array}$ \\
\hline 1 & $\mathrm{SnCl}_{2} \cdot 2 \mathrm{H}_{2} \mathrm{O}$ & $(0.1)$ & $\mathrm{MeOH}$ & r. t. & 96 & 82 \\
2 & $\mathrm{SnCl}_{2} \cdot 2 \mathrm{H}_{2} \mathrm{O}$ & $(0.1)$ & $\mathrm{EtOH}$ & r. t. & 96 & 88 \\
3 & $\mathrm{SnCl}_{2} \cdot 2 \mathrm{H}_{2} \mathrm{O}$ & $(0.1)$ & $i$-PrOH & r. t. & 96 & 96 \\
4 & $\mathrm{SnCl}_{2} \cdot 2 \mathrm{H}_{2} \mathrm{O}$ & $(0.2)$ & $i$-PrOH & r. t. & 96 & 97 \\
5 & $\mathrm{SnCl}_{2} \cdot 2 \mathrm{H}_{2} \mathrm{O}$ & $(0.5)$ & $i$-PrOH & r. t. & 96 & 90 \\
6 & $\mathrm{MnCl}_{2} \cdot 4 \mathrm{H}_{2} \mathrm{O}$ & $(0.1)$ & $i$-PrOH & r. t. & 120 & 93 \\
7 & $\mathrm{SrCl}_{2} \cdot 6 \mathrm{H}_{2} \mathrm{O}$ & $(0.1)$ & $i$-PrOH & r. t. & 120 & 70 \\
8 & $\mathrm{CoCl}_{2} \cdot 6 \mathrm{H}_{2} \mathrm{O}$ & $(0.1)$ & $i$-PrOH & r. t. & 120 & 79 \\
9 & $\mathrm{SnCl}_{2} \cdot 2 \mathrm{H}_{2} \mathrm{O}$ & $(0.1)$ & $i$-PrOH & reflux & 3 & 98 \\
10 & $\mathrm{CoCl}_{2} \cdot 6 \mathrm{H}_{2} \mathrm{O}$ & $(0.1)$ & $i$-PrOH & reflux & 5 & 98 \\
11 & $\mathrm{CrCl}_{3} \cdot 6 \mathrm{H}_{2} \mathrm{O}$ & $(0.1)$ & $i$-PrOH & reflux & 5 & 98 \\
\hline
\end{tabular}

For the $\mathrm{SnCl}_{2} \cdot 2 \mathrm{H}_{2} \mathrm{O}$, the increase of the catalyst loading did not improve the yields and the catalytic amount of $10 \mathrm{~mol} \%$ was chosen as the default load of the catalyst (entries 3, 4 and 5, respectively).

Although long reaction times were required, in general, reasonable to good yields were observed when $\mathrm{MnCl}_{2} \cdot 4 \mathrm{H}_{2} \mathrm{O}, \mathrm{SrCl}_{2} \cdot 6 \mathrm{H}_{2} \mathrm{O}, \mathrm{CoCl}_{2} \cdot 6 \mathrm{H}_{2} \mathrm{O}$ were used as catalysts in experiments carried out at room temperature (entries 6, 7 and 8). On the other hand, shorter reaction times of 3-5 h and refluxing conditions were enough to complete the consumption of the reagents when $\mathrm{SnCl}_{2} \cdot 2 \mathrm{H}_{2} \mathrm{O}, \mathrm{CoCl}_{2} \cdot 6 \mathrm{H}_{2} \mathrm{O}$ and $\mathrm{CrCl}_{2} \cdot 6 \mathrm{H}_{2} \mathrm{O}$ were used (entries 9, 10 and 11 , respectively).

Due to the easy availability, good stability and low cost, $\mathrm{SnCl}_{2} \cdot 2 \mathrm{H}_{2} \mathrm{O}$ was chosen as the default catalyst. Its use as a Lewis acid catalyst in the reaction of indoles $\mathbf{1 a - c}$ and ketones $\mathbf{2 a - e}$ afforded the respective $\beta$-indolylketones $\mathbf{3 b}$-m in reasonable to good yields (Scheme 2). The results are summarized in the Table 2.

As can be observed, the yields of adducts $\mathbf{3}$ were high, except for the cases where cyclohexenone was used as the Michael acceptor in the reactions with indole 1a (entries 4 and 9, respectively). In both cases, we were able to isolate variable amounts of two other side products, which showed a strong absorption in UV-light $(254 \mathrm{~nm})$. 
Table 2. Synthesis $\beta$-Indolylketones $\mathbf{3 b}$ - $\mathbf{m}$ under $\mathrm{SnCl}_{2} \cdot 2 \mathrm{H}_{2} \mathrm{O}$ catalyst at room temperature

\begin{tabular}{|c|c|c|c|c|c|c|c|c|c|}
\hline \multirow{2}{*}{ entry } & \multicolumn{3}{|c|}{ Indole } & \multicolumn{3}{|c|}{$\alpha, \beta$-Unsaturated ketone } & \multirow{2}{*}{ time (h) } & \multirow{2}{*}{ Product } & \multirow{2}{*}{ Yield (\%) } \\
\hline & & $\mathrm{R}^{1}$ & $\mathrm{R}^{2}$ & & $\mathrm{R}^{3}$ & $\mathrm{R}^{4}$ & & & \\
\hline 1 & $1 \mathrm{a}$ & $\mathrm{H}$ & $\mathrm{H}$ & $2 \mathbf{a}$ & $\mathrm{H}$ & Et & 48 & $3 \mathbf{b}$ & 96 \\
\hline 2 & 1a & $\mathrm{H}$ & $\mathrm{H}$ & $2 b$ & $\mathrm{C}_{5} \mathrm{H}_{11}$ & $\mathrm{Me}$ & 96 & $3 \mathrm{c}$ & 96 \\
\hline 3 & $1 \mathrm{a}$ & $\mathrm{H}$ & $\mathrm{H}$ & $2 c$ & cyclopentenone & & 78 & $3 d$ & 96 \\
\hline 4 & $1 \mathrm{a}$ & $\mathrm{H}$ & $\mathrm{H}$ & 2d & cyclohexenone & & 72 & $3 e$ & 40 \\
\hline 5 & $1 b$ & $\mathrm{MeO}$ & $\mathrm{H}$ & $2 e$ & $\mathrm{Me}$ & Et & 96 & $3 f$ & 98 \\
\hline 6 & $1 b$ & $\mathrm{MeO}$ & $\mathrm{H}$ & $2 \mathbf{a}$ & $\mathrm{H}$ & Et & 96 & $3 g$ & 80 \\
\hline 7 & $1 b$ & $\mathrm{MeO}$ & $\mathrm{H}$ & $2 \mathbf{b}$ & $\mathrm{C}_{5} \mathrm{H}_{11}$ & $\mathrm{Me}$ & 96 & $3 \mathbf{h}$ & 90 \\
\hline 8 & $1 \mathrm{~b}$ & $\mathrm{MeO}$ & $\mathrm{H}$ & $2 c$ & cyclopentenone & & 72 & $3 \mathbf{i}$ & 95 \\
\hline 9 & $1 b$ & $\mathrm{MeO}$ & $\mathrm{H}$ & 2d & cyclohexenone & & 32 & $3 \mathbf{j}$ & 41 \\
\hline 10 & $1 \mathrm{c}$ & $\mathrm{H}$ & $\mathrm{Me}$ & $2 e$ & $\mathrm{Me}$ & Et & 72 & $3 k$ & 88 \\
\hline 11 & 1c & $\mathrm{H}$ & $\mathrm{Me}$ & $2 a$ & $\mathrm{H}$ & Et & 48 & 31 & 97 \\
\hline 12 & $1 \mathrm{c}$ & $\mathrm{H}$ & $\mathrm{Me}$ & $2 c$ & cyclopentenone & & 48 & $3 m$ & 81 \\
\hline
\end{tabular}

We believe that the side products are generated by a second attack of an indole molecule to the initial $\beta$-indolylketone formed. Alternatively, a third molecule of indole could be added to the bis-indolyl adduct $\mathbf{3 n}$ and 3o to form the tris-indolyl adduct 3p and 3q, respectively, as reported recently by Shi et al..$^{23}$ (Figure 1).

All of the yields refer to isolated products after purification by column chromatography and the ${ }^{1} \mathrm{H}$ NMR, ${ }^{13} \mathrm{C}$ NMR and IR spectral data were in accordance with the proposed structures.

\section{Reactions of indoles with arylidene-phenylketones}

Next, we investigated the conjugated Friedel-Crafts reaction of indoles 1a-c with chalcones ${ }^{47} \mathbf{2 f}-\mathbf{i}$ in presence of $\mathrm{SnCl}_{2} \cdot 2 \mathrm{H}_{2} \mathrm{O}$ as the catalyst and $i-\mathrm{PrOH}$ as the solvent (Scheme 2).

In contrast with alkylidene-alkylketones, the chalcone $2 \mathbf{f}$ was not reactive with indole $1 \mathbf{a}$ at room temperature over $144 \mathrm{~h}$, even at $50 \mathrm{~mol} \%$ catalyst loading (cf. entries 1-3,
Table 3). On the other hand, the use of reflux conditions led to the formation of the desired adduct $3 \mathrm{r}$ after $5 \mathrm{~h}$ in good yield (entry 4). Other catalysts such as $\mathrm{MnCl}_{2} \cdot 4 \mathrm{H}_{2} \mathrm{O}$, $\mathrm{SrCl}_{2} \cdot 6 \mathrm{H}_{2} \mathrm{O}, \mathrm{CoCl}_{2} \cdot 6 \mathrm{H}_{2} \mathrm{O}$ and $\mathrm{CeCl}_{3} \cdot 7 \mathrm{H}_{2} \mathrm{O}$ were also effective (entries 5 and 8 ).

The chalcones $\mathbf{2 f}-\mathbf{i}$ were reacted under the previous reaction conditions and the $\beta$-indolylchalcones $\mathbf{3 r}$ - $\mathbf{z}$ were obtained in reasonable to good yields after purification by column chromatography (entries 9-15). The lower reactivity of chalcones may be attributed to their inferior electrophilicity as well as the increase of steric hindrance at the $\beta$-position of the ketone.

\section{Reactions of indoles with nitrostyrenes}

Due to the success of previous experiments we decided to investigate the CFC reactions of indoles 1a-c with nitroolefins $\mathbf{4 a - g}$. The nitroolefins are recognized as excellent Michael aceptors ${ }^{48,49}$ and their derivatives are important intermediates in organic synthesis
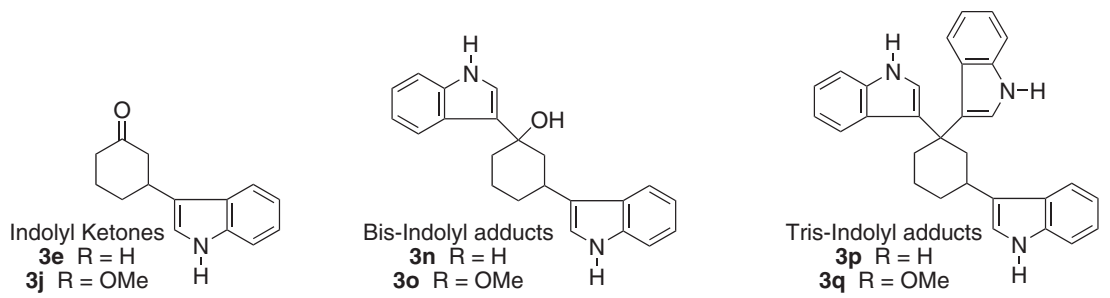

Figure 1. The possible structures of bis- and tris-indolyl adducts. 


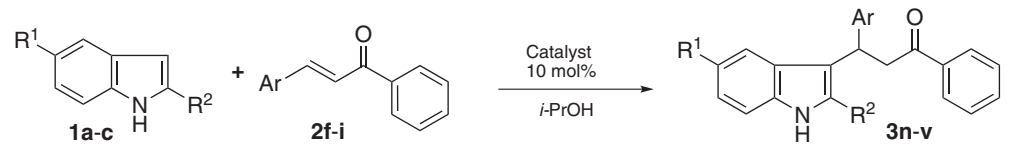

Scheme 2. Conjugated Friedel-Crafts reaction of indoles 1a-c with chalcones $\mathbf{2 f - i}$.

Table 3. Synthesis of $\beta$-indolylketones $\mathbf{3 r}-\mathbf{z}$ via Scheme 2

\begin{tabular}{|c|c|c|c|c|c|c|c|}
\hline \multirow{2}{*}{ entry } & \multicolumn{2}{|c|}{ Indole } & \multirow{2}{*}{$\begin{array}{c}\text { Olefin } \\
\text { Ar }\end{array}$} & \multirow{2}{*}{ Catalyst (equiv.) } & \multirow{2}{*}{ Temperature } & \multirow{2}{*}{ time (h) } & \multirow{2}{*}{3 / Yield (\%) } \\
\hline & $\mathrm{R}^{1}$ & $\mathrm{R}^{2}$ & & & & & \\
\hline 1 & $\mathrm{H}$ & $\mathrm{H}$ & $\mathrm{Ph}$ & $\mathrm{SnCl}_{2} \cdot 2 \mathrm{H}_{2} \mathrm{O}(0.1)$ & r. t. & 144 & $3 r /-$ \\
\hline 2 & $\mathrm{H}$ & $\mathrm{H}$ & $\mathrm{Ph}$ & $\mathrm{SnCl}_{2} \cdot 2 \mathrm{H}_{2} \mathrm{O}(0.2)$ & r. t. & 144 & $3 r /-$ \\
\hline 3 & $\mathrm{H}$ & $\mathrm{H}$ & $\mathrm{Ph}$ & $\mathrm{SnCl}_{2} \cdot 2 \mathrm{H}_{2} \mathrm{O}(0.5)$ & r. t. & 144 & $3 r /-$ \\
\hline 4 & $\mathrm{H}$ & $\mathrm{H}$ & $\mathrm{Ph}$ & $\mathrm{SnCl}_{2} \cdot 2 \mathrm{H}_{2} \mathrm{O}(0.1)$ & reflux & 5 & $3 r / 96$ \\
\hline 5 & $\mathrm{H}$ & $\mathrm{H}$ & $\mathrm{Ph}$ & $\mathrm{MnCl}_{2} \cdot 4 \mathrm{H}_{2} \mathrm{O}(0.1)$ & reflux & 8 & $3 \mathbf{r} / 60$ \\
\hline 6 & $\mathrm{H}$ & $\mathrm{H}$ & $\mathrm{Ph}$ & $\mathrm{SrCl}_{2} \cdot 6 \mathrm{H}_{2} \mathrm{O}(0.1)$ & reflux & 5 & $3 \mathbf{r} / 60$ \\
\hline 7 & $\mathrm{H}$ & $\mathrm{H}$ & $\mathrm{Ph}$ & $\mathrm{CoCl}_{2} \cdot 6 \mathrm{H}_{2} \mathrm{O}(0.1)$ & reflux & 5 & $3 \mathbf{r} / 85$ \\
\hline 8 & $\mathrm{H}$ & $\mathrm{H}$ & $\mathrm{Ph}$ & $\mathrm{CeCl}_{3} \cdot 7 \mathrm{H}_{2} \mathrm{O}(0.1)$ & reflux & 5 & $3 \mathbf{3 r} / 98$ \\
\hline 9 & $\mathrm{H}$ & $\mathrm{H}$ & 1-naftyl & $\mathrm{SnCl}_{2} \cdot 2 \mathrm{H}_{2} \mathrm{O}(0.1)$ & reflux & 5 & $3 s / 60$ \\
\hline 10 & $\mathrm{H}$ & $\mathrm{H}$ & 4- $\mathrm{MeO}-\mathrm{C}_{6} \mathrm{H}_{4}$ & $\mathrm{SnCl}_{2} \cdot 2 \mathrm{H}_{2} \mathrm{O}(0.1)$ & reflux & 5 & $3 t / 80$ \\
\hline 11 & $\mathrm{H}$ & $\mathrm{H}$ & 2-thienyl & $\mathrm{SnCl}_{2} \cdot 2 \mathrm{H}_{2} \mathrm{O}(0.1)$ & reflux & 5 & $3 u / 74$ \\
\hline 12 & $\mathrm{MeO}$ & $\mathrm{H}$ & $\mathrm{Ph}$ & $\mathrm{SnCl}_{2} \cdot 2 \mathrm{H}_{2} \mathrm{O}(0.1)$ & reflux & 5 & $3 v / 96$ \\
\hline 13 & $\mathrm{MeO}$ & $\mathrm{H}$ & 1-naftyl & $\mathrm{SnCl}_{2} \cdot 2 \mathrm{H}_{2} \mathrm{O}(0.1)$ & reflux & 5 & $3 w / 70$ \\
\hline 14 & $\mathrm{MeO}$ & $\mathrm{H}$ & $4-\mathrm{MeO}-\mathrm{C}_{6} \mathrm{H}_{4}$ & $\mathrm{SnCl}_{2} \cdot 2 \mathrm{H}_{2} \mathrm{O}(0.1)$ & reflux & 5 & $3 \mathbf{x} / 95$ \\
\hline 15 & $\mathrm{MeO}$ & $\mathrm{H}$ & 2-thienyl & $\mathrm{SnCl}_{2} \cdot 2 \mathrm{H}_{2} \mathrm{O}(0.1)$ & reflux & 5 & 3y / 92 \\
\hline 16 & $\mathrm{H}$ & $\mathrm{Me}$ & $\mathrm{Ph}$ & $\mathrm{SnCl}_{2} \cdot 2 \mathrm{H}_{2} \mathrm{O}(0.1)$ & reflux & 5 & $3 z / 87$ \\
\hline
\end{tabular}

due to the synthetic versatility of the nitro-group as precursor of other organic functionalities..$^{50-52}$ First, we investigated the reaction of indole 1a wiyh nitrostyrene 4a carried out under the reactions conditions described above $(i-\mathrm{PrOH}$, room temperature and $10 \mathrm{~mol} \%$ of $\mathrm{SnCl}_{2} \cdot 2 \mathrm{H}_{2} \mathrm{O}$ ). Unfortunately, no product was formed even after 6 days. A second experiment was carried out under reflux conditions over $5 \mathrm{~h}$ and total consumption of the starting materials was observed. The TLC analysis revealed the presence of very polar mixture of byproducts ${ }^{53}$ which were not identified. After purification by chromatography, product $\mathbf{5 a}$ was obtained in only $54 \%$ yield (Scheme 3 ).

With the aim of improving the chemo-selectivity of the reaction and the yields of the products, the catalyst was changed to $\mathrm{CoCl}_{2} \cdot 6 \mathrm{H}_{2} \mathrm{O}$, which had been successfully applied previously (see Table 1 entry 10 and Table 3, entry 7 , respectively).

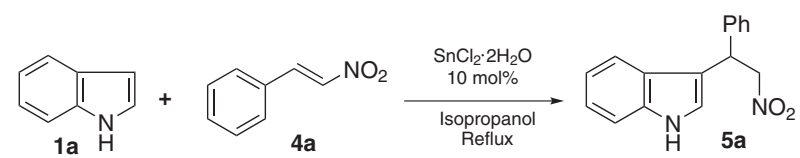

Scheme 3. Conjugated Friedel-Crafts reaction of indole with nitrostyrene 4a.
The reaction was carried out in $i$-PrOH under reflux conditions for $2 \mathrm{~h}$, when total consumption of the starting material was observed by TLC. After purification by chromatography, the nitro-adduct $\mathbf{5 a}$ was isolated in $\mathbf{9 5 \%}$ yield (Table 4, entry 1 ).

At this point we decided to investigate a set of different solvents. The experiments were carried out in polar protic

Table 4. Synthesis of adduct $5 \mathbf{a}$ promoted by $\mathrm{CoCl}_{2} \cdot 6 \mathrm{H}_{2} \mathrm{O}$ under different solvents

\begin{tabular}{lllllc} 
entry & Catalyst & Solvent & $\begin{array}{c}\text { time } \\
\text { (h) }\end{array}$ & Temperature & $\begin{array}{c}\text { Yield } \\
(\%)\end{array}$ \\
\hline 1 & $\mathrm{CoCl}_{2} \cdot 6 \mathrm{H}_{2} \mathrm{O}$ & $i-\mathrm{PrOH}$ & 2 & reflux & 95 \\
2 & $\mathrm{CoCl}_{2} \cdot 6 \mathrm{H}_{2} \mathrm{O}$ & $\mathrm{MeOH}$ & 2 & reflux & 72 \\
4 & $\mathrm{CoCl}_{2} \cdot 6 \mathrm{H}_{2} \mathrm{O}$ & $\mathrm{EtOH}$ & 2 & reflux & 73 \\
5 & $\mathrm{CoCl}_{2} \cdot 6 \mathrm{H}_{2} \mathrm{O}$ & $\mathrm{THF}_{2}$ & 2 & reflux & 58 \\
6 & $\mathrm{CoCl}_{2} \cdot 6 \mathrm{H}_{2} \mathrm{O}$ & $\mathrm{CH}_{3} \mathrm{CN}$ & 2 & reflux & 55 \\
7 & $\mathrm{CoCl}_{2} \cdot 6 \mathrm{H}_{2} \mathrm{O}$ & $\mathrm{CH}_{2} \mathrm{Cl}$ & 2 & reflux & 0 \\
\hline $\mathrm{CoCl}_{2} \cdot 6 \mathrm{H}_{2} \mathrm{O}$ & $\mathrm{C}_{6} \mathrm{H}_{6}$ & 2 & reflux & 71 \\
\hline
\end{tabular}


solvents such as $\mathrm{MeOH}$ and $\mathrm{EtOH}$; polar non protic solvents such as THF, $\mathrm{CH}_{3} \mathrm{CN}$ and $\mathrm{CH}_{2} \mathrm{Cl}_{2}$ and in the non polar non protic solvent such as $\mathrm{C}_{6} \mathrm{H}_{6}$. The results are shown in Table 4.

As can be seen, the best result was achieved when the reaction was carried out under reflux of $i-\mathrm{PrOH}$ (Table 4, entry 1). On the other hand, the reaction did not proceed in $\mathrm{CH}_{2} \mathrm{Cl}_{2}$ (entry 6). It should be noted that the yield of product 5a in the reaction performed in $\mathrm{C}_{6} \mathrm{H}_{6}$ was similar to those obtained in $\mathrm{MeOH}$ or EtOH (entries 7, 2 and 3, respectively).

\section{Solventless CFC reactions of indoles with nitrostyrenes}

Due to the constant search for environmentally-friendly protocols that avoid the collateral production of wastes in the synthetic organic chemistry, many efforts have been made to develop organic reactions in solventless conditions. . $^{54-56}$

Therefore, we decided to investigate the viability of $\mathrm{CFC}$ reaction of indoles and nitrostyrenes catalyzed by metal halide hydrates under solventless conditions. The results are summarized in Table 5 .

The best result was achieved when $\mathrm{CoCl}_{2} \cdot 6 \mathrm{H}_{2} \mathrm{O}$ was employed over $15 \mathrm{~min}$ at $100{ }^{\circ} \mathrm{C}$ (entry 1 ). Good yields were also achieved with $\mathrm{CrCl}_{3} \cdot 6 \mathrm{H}_{2} \mathrm{O}$ and $\mathrm{CeCl}_{3} \cdot 7 \mathrm{H}_{2} \mathrm{O}$ with little increase of the reaction times (entries 2 and 3). The employment of $\mathrm{MnCl}_{2} \cdot 4 \mathrm{H}_{2} \mathrm{O}, \mathrm{NiCl}_{2} \cdot 6 \mathrm{H}_{2} \mathrm{O}$ or $\mathrm{SrCl}_{2} \cdot 6 \mathrm{H}_{2} \mathrm{O}$ showed lower activity and an increase of the reaction time was needed to complete the consumption of the reagents (entries 4-6, respectively). In presence of $\mathrm{SnCl}_{2} \cdot 2 \mathrm{H}_{2} \mathrm{O}$, even over prolonged time, poor yield was obtained (entry 7).

To explore the generality of the solventless conjugated Friedel-Crafts reaction, we synthesized a set of nitrostyrene derivatives through a methodology reported in the literature ${ }^{57}$ Reaction of nitromethane with various aromatic aldehydes in the presence of ammonium acetate under heating (Scheme 4) afforded the respective nitroolefins 4a-g in 65-89\% yields, after recrystallization (Figure 2).

The solventless CFC reactions were carried out under the same conditions disclosed above and afforded the respective nitro adducts $\mathbf{5 b} \mathbf{b}-\mathbf{q}$. The results are summarized in Table 6.
Table 5. Synthesis of adduct 5a under different catalysts in solventless conditions

\begin{tabular}{lllccc} 
Entry & Catalyst & Condition & $\begin{array}{c}\text { time } \\
(\mathrm{h})\end{array}$ & $\begin{array}{c}\mathrm{Temp} \\
\left({ }^{\circ} \mathrm{C}\right)\end{array}$ & $\begin{array}{c}\text { Yield } \\
(\%)\end{array}$ \\
\hline 1 & $\mathrm{CoCl}_{2} \cdot 6 \mathrm{H}_{2} \mathrm{O}$ & solventless & 0,25 & 100 & 97 \\
2 & $\mathrm{CrCl}_{3} \cdot 6 \mathrm{H}_{2} \mathrm{O}$ & solventless & 0,50 & 100 & 81 \\
3 & $\mathrm{CeCl}_{3} \cdot 7 \mathrm{H}_{2} \mathrm{O}$ & solventless & 0,50 & 100 & 93 \\
4 & $\mathrm{MnCl}_{2} \cdot 4 \mathrm{H}_{2} \mathrm{O}$ & solventless & 2 & 100 & 95 \\
5 & $\mathrm{NiCl}_{2} \cdot 6 \mathrm{H}_{2} \mathrm{O}$ & solventless & 8 & 100 & 95 \\
6 & $\mathrm{SrCl}_{2} \cdot 6 \mathrm{H}_{2} \mathrm{O}$ & solventless & 12 & 100 & 96 \\
7 & $\mathrm{SnCl}_{2} \cdot 2 \mathrm{H}_{2} \mathrm{O}$ & solventless & 120 & 100 & 57 \\
\hline
\end{tabular}

In general, all reactions afforded the nitro-adducts in good yields. The reactivity of the nitroolefin $\mathbf{4 d}$ was lower for both indoles 1a and $\mathbf{1 b}$ (entries 3 and 10, respectively). This may be due to the strong electron releasing effect of the $\mathrm{N}, \mathrm{N}$-dimethyl group attached at 4-position of the aromatic ring. Also, the steric hindrance caused by the bulky naphtyl group could be responsible for de increase of the reaction times with the indoles $\mathbf{1 a}$ and $\mathbf{1 c}$ and the nitroolefin $\mathbf{4 f}$ (entry 5 and 15 , respectively).

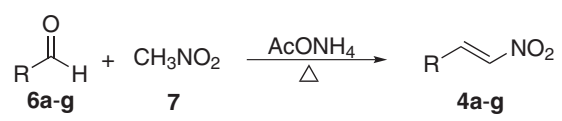

Scheme 4. Synthesis of nitroolefins 4a-g.

Synthesis of $\beta$-carboline derivative via Pictet-Spengler reaction

To demonstrate the synthetic utility of the nitro adduct intermediate 5a we performed the transformation of the nitro group into an amino group by reduction in the presence of $\mathrm{NH}_{4} \mathrm{OAc}$ and catalytic amounts of $\mathrm{Pd} / \mathrm{C} .^{58}$ The triptamine derivative $\mathbf{8}$ was obtained in $90 \%$ yield as a crude product.

After the treatment of compound $\mathbf{8}$ with benzaldehyde under the Pictet-Spengler reaction conditions, the
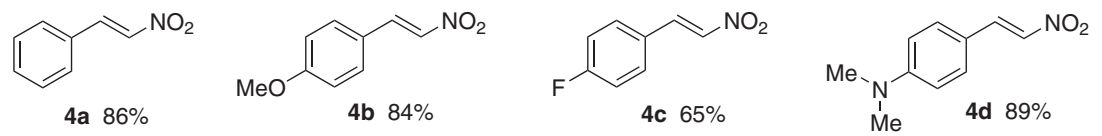<smiles>COc1ccc(/C=C/[N+](=O)[O-])cc1OC</smiles><smiles>O=[N+]([O-])C=Cc1cccc2ccccc12</smiles>

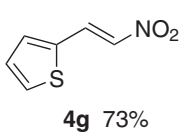

Figure 2. Structures of nitroolefins $\mathbf{4 a - g}$. 
Table 6. Synthesis of adduct $\mathbf{5 b}$-q by CFC reaction under solventless conditions

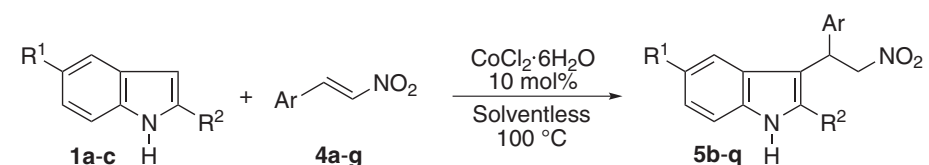
1a-c $\stackrel{+}{\mathrm{H}} \quad \mathbf{4 a - g} \quad 100^{\circ} \mathrm{C} \quad \mathbf{5 b - q} \stackrel{亡}{\mathrm{H}}$

\begin{tabular}{|c|c|c|c|c|c|c|c|c|}
\hline \multirow[t]{2}{*}{ entry } & & \multicolumn{2}{|l|}{ Indole } & \multicolumn{2}{|c|}{ Nitroolefin } & \multirow[t]{2}{*}{ time (min) } & \multirow[t]{2}{*}{ Product } & \multirow[t]{2}{*}{ Yield (\%) } \\
\hline & & $\mathrm{R}^{1}$ & $\mathrm{R}^{2}$ & & $\mathrm{Ar}$ & & & \\
\hline 1 & $1 \mathrm{a}$ & $\mathrm{H}$ & $\mathbf{H}$ & $4 b$ & $4-\mathrm{MeO}-\mathrm{C}_{6} \mathrm{H}_{4}$ & 15 & $5 b$ & 92 \\
\hline 2 & $1 \mathrm{a}$ & $\mathrm{H}$ & $\mathrm{H}$ & $4 c$ & 4-F- $\mathrm{C}_{6} \mathrm{H}_{4}$ & 15 & $5 c$ & 97 \\
\hline 3 & $1 \mathrm{a}$ & $\mathrm{H}$ & $\mathrm{H}$ & $4 d$ & $4-N, N(\mathrm{Me})_{2}-\mathrm{C}_{6} \mathrm{H}_{4}$ & 60 & $5 d$ & 98 \\
\hline 4 & 1a & $\mathrm{H}$ & $\mathrm{H}$ & $4 e$ & $3,4-(\mathrm{MeO})-\mathrm{C}_{6} \mathrm{H}_{3}$ & 15 & $5 e$ & 90 \\
\hline 5 & $1 \mathrm{a}$ & $\mathrm{H}$ & $\mathrm{H}$ & $4 f$ & 1-naphtyl & 30 & $5 f$ & 97 \\
\hline 6 & $1 \mathrm{a}$ & $\mathrm{H}$ & $\mathrm{H}$ & $4 g$ & 2-thienyl & 15 & $5 g$ & 98 \\
\hline 7 & $1 b$ & $\mathrm{MeO}$ & $\mathrm{H}$ & $4 a$ & $\mathrm{Ph}$ & 15 & $5 h$ & 95 \\
\hline 8 & $1 b$ & $\mathrm{MeO}$ & $\mathrm{H}$ & $4 b$ & $4-\mathrm{MeO}-\mathrm{C}_{6} \mathrm{H}_{4}$ & 15 & $5 i$ & 86 \\
\hline 9 & $1 b$ & $\mathrm{MeO}$ & $\mathrm{H}$ & $4 c$ & 4-F- $\mathrm{C}_{6} \mathrm{H}_{4}$ & 15 & $5 j$ & 96 \\
\hline 10 & $1 b$ & $\mathrm{MeO}$ & $\mathrm{H}$ & $4 d$ & $4-N, N(\mathrm{Me})_{2}-\mathrm{C}_{6} \mathrm{H}_{4}$ & 30 & $5 \mathbf{k}$ & 87 \\
\hline 11 & $1 b$ & $\mathrm{MeO}$ & $\mathrm{H}$ & $4 e$ & $3,4-(\mathrm{MeO})-\mathrm{C}_{6} \mathrm{H}_{3}$ & 15 & 51 & 98 \\
\hline 12 & $1 b$ & $\mathrm{MeO}$ & $\mathrm{H}$ & $4 f$ & 1-naphtyl & 15 & $5 \mathrm{~m}$ & 98 \\
\hline 13 & $1 b$ & $\mathrm{MeO}$ & $\mathrm{H}$ & $4 \mathrm{~g}$ & 2-thienyl & 15 & $5 n$ & 93 \\
\hline 14 & $1 \mathrm{c}$ & $\mathrm{H}$ & $\mathrm{Me}$ & $4 a$ & $\mathrm{Ph}$ & 15 & 50 & 94 \\
\hline 15 & $1 \mathrm{c}$ & $\mathrm{H}$ & $\mathrm{Me}$ & $4 f$ & 1-naphtyl & 30 & $5 p$ & 93 \\
\hline 16 & $1 \mathrm{c}$ & $\mathrm{H}$ & $\mathrm{Me}$ & $4 g$ & 2-thienyl & 15 & $5 q$ & 98 \\
\hline
\end{tabular}

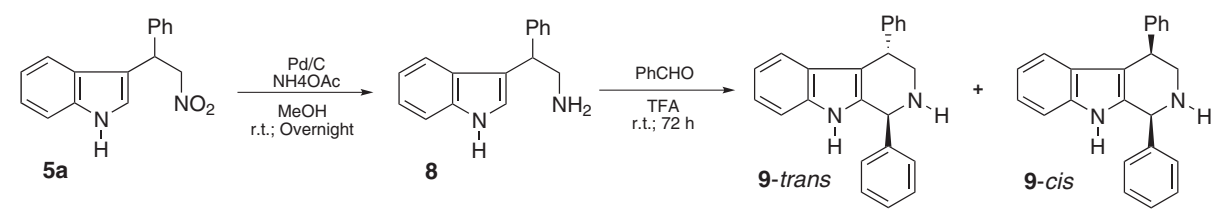

Scheme 5. Synthesis of 1,2,3,4-tetrahydro- $\beta$-carbolines.

$\beta$-Carboline derivative 9 was isolated as a diastereomeric mixture of $87: 13$ ratios. After purification by chromatography, the compound 9-trans was isolated in $70 \%$ yield. The relative trans configuration of the major distereoisomer was inferred by comparison of the ${ }^{1} \mathrm{H}$ NMR data with the reported literature (Scheme 5) ${ }^{59}$

\section{Conclusions}

We have demonstrated that many metal halides hydrates are able to catalyze the conjugate Friedel-Crafts reaction of indoles and activated olefins in good to excellent yields. The alcoholic solvents were superior and $i-\mathrm{PrOH}$ was found to be the best choice.

For the reactions with aliphatic enones longer reaction times were needed for those reaction carried out at room temperature, whereas shorter times were observed under refluxing conditions. The metal halide hydrates
$\mathrm{SnCl}_{2} \cdot 2 \mathrm{H}_{2} \mathrm{O}, \mathrm{MnCl}_{2} \cdot 4 \mathrm{H}_{2} \mathrm{O}, \mathrm{CrCl}_{2} \cdot 6 \mathrm{H}_{2} \mathrm{O}, \mathrm{CoCl}_{2} \cdot 6 \mathrm{H}_{2} \mathrm{O}$ were all effective.

Chalcones as Michael acceptors afforded the desired adducts only under refluxing conditions using $\mathrm{SnCl}_{2} \cdot 2 \mathrm{H}_{2} \mathrm{O}$ as catalysts.

For the reaction with of nitroolefins, refluxing conditions are imperative under the liquid phase. Here, the alcoholic $i$-PrOH was superior. On the other hand, $\mathrm{SnCl}_{2} \cdot 2 \mathrm{H}_{2} \mathrm{O}$ leads to the formation of several byproducts and therefore it was abandoned. The best results were achieved in the presence of $\mathrm{CoCl}_{2} \cdot 6 \mathrm{H}_{2} \mathrm{O}$. These reactions were also investigated in the absence of solvents under $100{ }^{\circ} \mathrm{C}$. The metal halide hydrates $\mathrm{CoCl}_{2} \cdot 6 \mathrm{H}_{2} \mathrm{O}, \mathrm{CrCl}_{3} \cdot 6 \mathrm{H}_{2} \mathrm{O}, \mathrm{CeCl}_{3} \cdot 7 \mathrm{H}_{2} \mathrm{O}$, $\mathrm{MnCl}_{2} \cdot 4 \mathrm{H}_{2} \mathrm{O}, \mathrm{NiCl}_{2} \cdot 6 \mathrm{H}_{2} \mathrm{O}$ and $\mathrm{SrCl}_{2} \cdot 6 \mathrm{H}_{2} \mathrm{O}$ were all effective in the reaction, but with different reaction times. The $\mathrm{CoCl}_{2} \cdot 6 \mathrm{H}_{2} \mathrm{O}, \mathrm{CrCl}_{3} \cdot 6 \mathrm{H}_{2} \mathrm{O}$ and $\mathrm{CeCl}_{3} \cdot 7 \mathrm{H}_{2} \mathrm{O}$ were the most effective catalysts. In summary, we have demonstrated the use of cheaper and easy to handle metal halide hydrates 
as Lewis acids catalysts in the conjugated Friedel-Crafts reaction under enviromentally benign alcoholic solvents or under eco-friendly solventless protocols.

\section{Experimental}

All reactions were carried out under air. The chalcones ${ }^{47}$ (2f-i) and the nitrostyrenes ${ }^{57}$ (4a-g) were prepared according to the literature. All other reagents and solvents were purchased from commercial suppliers and used without further purification except hexane which was distilled prior to use. The ${ }^{1} \mathrm{H}$ and ${ }^{13} \mathrm{C}$ NMR spectra were recorded in $\mathrm{CDCl}_{3}$ on a Varian VNMRS spectrometer at $300 \mathrm{MHz}$ and $75 \mathrm{MHz}$, respectively. The chemical shifts $(\delta)$ are reported in ppm relative to TMS $(0.00 \mathrm{ppm})$ for ${ }^{1} \mathrm{H}$ NMR and to the central line of $\mathrm{CDCl}_{3}(77.0 \mathrm{ppm})$ for ${ }^{13} \mathrm{C}$ NMR. Coupling constants $J$ are reported in Hertz (Hz). IR spectra were recorded on a FTIR-Varian 640-IR spectrometer. Melting points were measured on an Olympus BX41 microscope equipped with a Mettler-Toledo FP82HT hot stage (Mettler-Toledo FP90 controller) and are uncorrected. Reactions were monitored using thin layer chromatography (TLC) carried out on Merck silica gel $60 \mathrm{~F}_{254}$ precoated aluminium plates. The visualization was achieved under UV light $(254 \mathrm{~nm})$ or staining with $\mathrm{I}_{2}$. Chromatographic separations were achieved on silica gel columns (70-230 mesh, Aldrich) using gradient of hexanes/ethyl acetate as eluent.

General procedure for the synthesis of $\beta$-indolylketones 3a-m

In a $25 \mathrm{~mL}$ round-bottom flask, equipped with magnetic stirrer were added the indoles $\mathbf{1 a - c}(1.0 \mathrm{mmol})$, the $\alpha, \beta$-unsaturated ketones $2 \mathbf{a}$-e $(1.1 \mathrm{mmol})$ and $\mathrm{SnCl}_{2} \cdot 2 \mathrm{H}_{2} \mathrm{O}$ $(0.1 \mathrm{mmol})$ in $i-\mathrm{PrOH}(2 \mathrm{~mL})$. The reaction mixture was stirred at room temperature for the appropriate time shown in the Table 2. After the completion of the reaction, monitored by TLC, the solvent was removed under vacuum and the crude products 3a-m were purified by column chromatography using a gradient of hexanes/ethyl acetate as eluent.

5-(1H-Indol-3-yl)hexan-3-one (3a) $:{ }^{60} 96 \%$, viscous brown oil; ' ${ }^{1} \mathrm{H}$ NMR $\left(300 \mathrm{MHz}, \mathrm{CDCl}_{3}\right) \delta 8.25$ (br s, $\left.1 \mathrm{H}\right)$, $7.63(\mathrm{~m}, 1 \mathrm{H}), 7.28(\mathrm{~m}, 1 \mathrm{H}), 7.18-7.04(\mathrm{~m}, 2 \mathrm{H}), 6.82(\mathrm{~s}$, $1 \mathrm{H}), 3.62(\mathrm{~m}, 1 \mathrm{H}), 2.86(\mathrm{dd}, J 15.9$ and $6.3 \mathrm{~Hz}, 1 \mathrm{H}), 2.64$ (dd, $J 15.9$ and $8.1 \mathrm{~Hz}, 1 \mathrm{H}), 2.31$ (q, J 7.2 Hz, 2H), 1.34 (d, $J$ 6,9 Hz, 3H), 0.96 (t, $J 7.2 \mathrm{~Hz}, 3 \mathrm{H}) ;{ }^{13} \mathrm{C}$ NMR $(75 \mathrm{MHz}$, $\left.\mathrm{CDCl}_{3}\right) \delta 211.8,136.4,126.0,121.7,120.5,120.2,118.9$, $118.9,111.3,50.1,36.3,26.9,21.1,7,6 ; \mathrm{IR} v_{\max } / \mathrm{cm}^{-1}$ (neat, film) $3411(\mathrm{NH}), 2971,1705(\mathrm{C}=\mathrm{O}), 1458,1375,1354$, 1341, 743 .
1-(1H-Indol-3-yl)pentan-3-one (3b): ${ }^{61} 96 \%$, brown solid, mp 86-87 ${ }^{\circ} \mathrm{C}$; ${ }^{1} \mathrm{H}$ NMR (300 MHz, $\mathrm{CDCl}_{3}$ ) $\delta 8.20$ (br s, 1H), $7.56(\mathrm{~d}, J 7.8 \mathrm{~Hz}, 1 \mathrm{H}), 7.27(\mathrm{~d}, J 8.1 \mathrm{~Hz}, 1 \mathrm{H})$, 7.20-7.05 (m, 2H), $6.89(\mathrm{~d}, J 1.8 \mathrm{~Hz}, 1 \mathrm{H}), 3.03(\mathrm{t}, J 7.2$ $\mathrm{Hz}, 2 \mathrm{H}), 2.77$ (t, $J 7.2 \mathrm{~Hz}, 2 \mathrm{H}), 2.26$ (q, J $7.5 \mathrm{~Hz}, 2 \mathrm{H}$ ), $1.01(\mathrm{t}, J 7.5 \mathrm{~Hz}, 3 \mathrm{H}) ;{ }^{13} \mathrm{C}$ NMR $\left(75 \mathrm{MHz}, \mathrm{CDCl}_{3}\right) \delta 211.8$, 136.2, 127.0, 121.8, 121.5, 119.0, 118.5, 114.9, 111.2, 42.7, 35.9, 19.3, 7.7; IR $v_{\max } / \mathrm{cm}^{-1}(\mathrm{KBr}) 3323(\mathrm{NH}), 1704$ $(\mathrm{C}=\mathrm{O}), 1458,736$.

4-(1H-Indol-3-yl)nonan-2-one (3c): $:^{60} 96 \%$, viscous brown oil; ${ }^{1} \mathrm{H}$ NMR $\left(300 \mathrm{MHz}, \mathrm{CDCl}_{3}\right) \delta 8.09$ (br s, 1H), $7.64(\mathrm{~d}, J 7.8 \mathrm{~Hz}, 1 \mathrm{H}), 7.31(\mathrm{~d}, J 7.8 \mathrm{~Hz}, 1 \mathrm{H}), 7.21-7.05$ (m, 2H), $6.91(\mathrm{~d}, J 2.4 \mathrm{~Hz}, 1 \mathrm{H}), 3.43(\mathrm{~m}, 1 \mathrm{H}), 2.88$ (dd, $J 15.9$ and $7.5 \mathrm{~Hz}, 1 \mathrm{H}), 2.78(\mathrm{dd}, J 15.9$ and $6.9 \mathrm{~Hz}, 1 \mathrm{H}$ ), 2.00 (s, 3H), 1.62-1.82 (m, 2H), 1.23 (br s, 6H), 0.81 (br s, 3H); ${ }^{13} \mathrm{C}$ NMR (75 MHz, $\left.\mathrm{CDCl}_{3}\right) \delta 209.2,136.5,126.5$, $121.8,121.2,119.2,119.1,118.8,111.3,50.2,35.8,32.9$, 31.8, 30.4, 27.2, 22.5, 14.0; IR $v_{\max } / \mathrm{cm}^{-1}$ (neat, film) 3412 (NH), 2956, 2928, 2856, 1707 (C=O), 1458, 1357, 742 .

3-(1H-Indol-3-yl)-cyclopentanone (3d): ${ }^{61} 96 \%$, viscous brown oil; ${ }^{1} \mathrm{H}$ NMR $\left(300 \mathrm{MHz}, \mathrm{CDCl}_{3}\right) \delta 8.34$ (br s, $1 \mathrm{H}), 7.60(\mathrm{~d}, J 7.8 \mathrm{~Hz}, 1 \mathrm{H}), 7.32$ (dd, $J 7.8$ and $0.9 \mathrm{~Hz}$, 1H), $7.19(\mathrm{dt}, J 7.5$ and $1.2 \mathrm{~Hz}, 1 \mathrm{H}), 7.12(\mathrm{dt}, J 7.5$ and $1.2 \mathrm{~Hz}, 1 \mathrm{H}), 6.88(\mathrm{~d}, J 1.8 \mathrm{~Hz}, 1 \mathrm{H}), 3.65(\mathrm{~m}, 1 \mathrm{H}), 2.72(\mathrm{dd}$, $J 18.0$ and $7.2 \mathrm{~Hz}, 1 \mathrm{H}), 2.65-2.17(\mathrm{~m}, 4 \mathrm{H}), 2.17-1.98(\mathrm{~m}$, $1 \mathrm{H}) ;{ }^{13} \mathrm{C}$ NMR $\left(75 \mathrm{MHz}, \mathrm{CDCl}_{3}\right) \delta 220.0,136.6,126.5$, 122.1, 120.0, 119.2, 118.9, 118.2, 111.3, 45.2, 38.1, 33.6, 29.7; IR $v_{\text {max }} / \mathrm{cm}^{-1}$ (neat, film) $3410(\mathrm{NH}), 1734(\mathrm{C}=\mathrm{O})$, 1458, 1401, 1230, 1169, 744.

3-(1H-Indol-3-yl)-cyclohexenone (3e ): ${ }^{60} 40 \%$, viscous brown oil; ${ }^{1} \mathrm{H}$ NMR $\left(300 \mathrm{MHz}, \mathrm{CDCl}_{3}\right) \delta 8.18$ (br s, $1 \mathrm{H}$ ), $7.61(\mathrm{~d}, J 7.8 \mathrm{~Hz}, 1 \mathrm{H}), 7.34(\mathrm{~d}, J 8.1 \mathrm{~Hz}, 1 \mathrm{H}), 7.20(\mathrm{t}$, $J 7.5 \mathrm{~Hz}, 1 \mathrm{H}), 7.11(\mathrm{t}, J 7.5 \mathrm{~Hz}, 1 \mathrm{H}), 6.94(\mathrm{~d}, J 1.5 \mathrm{~Hz}$, $1 \mathrm{H}), 3.43(\mathrm{~m}, 1 \mathrm{H}), 2.92-2.72(\mathrm{~m}, 1 \mathrm{H}), 2.72-2.57(\mathrm{~m}, 1 \mathrm{H})$, 2.57-2.33 (m, 2H), 2.33-2.16 (m, 1H), 2.16-1.62 (m, 3H); ${ }^{13} \mathrm{C}$ NMR $\left(75 \mathrm{MHz}, \mathrm{CDCl}_{3}\right) \delta 212.1,136.4,126.0,122.1$, 120.4, 119.5, 119.2, 118.9, 111.3, 48.0, 41.5, 35.9, 31.6, 24.8; IR $v_{\text {max }} / \mathrm{cm}^{-1}$ (neat, film) $3411(\mathrm{NH}), 2971,1705(\mathrm{C}=\mathrm{O})$, 1458, 1375, 1354, 1340, 743.

5-(5-Methoxy-1H-indol-3-yl)hexan-3-one (3f): ${ }^{62} 98 \%$, viscous brown oil; ${ }^{1} \mathrm{H}$ NMR $\left(300 \mathrm{MHz}, \mathrm{CDCl}_{3}\right) \delta 8.29$ (br s, 1H), $7.16(\mathrm{~d}, J 8.7 \mathrm{~Hz}, 1 \mathrm{H}), 7.06(\mathrm{~d}, J 2.1 \mathrm{~Hz}, 1 \mathrm{H}), 6.86$ $(\mathrm{d}, J 2.1 \mathrm{~Hz}, 1 \mathrm{H}), 6.83(\mathrm{dd}, J 8.7$ and $2.1 \mathrm{~Hz}, 1 \mathrm{H}), 3.84(\mathrm{~s}$, $3 \mathrm{H}), 3.59(\mathrm{~m}, 1 \mathrm{H}), 2.86(\mathrm{dd}, J 15.9$ and $6.0 \mathrm{~Hz}, 1 \mathrm{H}), 2.65$ (dd, $J 15.9$ and $8.4 \mathrm{~Hz}, 1 \mathrm{H}), 2.32(\mathrm{q}, J 7.2 \mathrm{~Hz}, 2 \mathrm{H}), 1.34$ (d, $J 6.9 \mathrm{~Hz}, 3 \mathrm{H}), 0,98(\mathrm{t}, J 7.2 \mathrm{~Hz}, 3 \mathrm{H}) ;{ }^{13} \mathrm{C}$ NMR $(75 \mathrm{MHz}$, $\left.\mathrm{CDCl}_{3}\right) \delta 211.6 ; 153.5,131.6,126.4 ; 121.0 ; 120.4,112.0$, 
$111.7,100.9,55.8,50.0,36.3,26.8,21.1,7.5 ; \mathrm{IR} v_{\max } / \mathrm{cm}^{-1}$ (neat, film) $3407(\mathrm{NH}), 3383,2964,1705(\mathrm{C}=\mathrm{O})$.

1-(5-Methoxy-1H-indol-3-yl)-pentan-3-one (3g): $80 \%$, brown solid, mp $94-95{ }^{\circ} \mathrm{C}$; ${ }^{1} \mathrm{H}$ NMR $(300 \mathrm{MHz}$, $\left.\mathrm{CDCl}_{3}\right) \delta 7.89(\mathrm{br} \mathrm{s}, 1 \mathrm{H}), 7.24(\mathrm{~d}, J 8.7 \mathrm{~Hz}, 1 \mathrm{H}), 7.02(\mathrm{~d}$, $J 2.4 \mathrm{~Hz}, 1 \mathrm{H}), 6.96(\mathrm{~d}, J 2.4 \mathrm{~Hz}, 1 \mathrm{H}), 6.85(\mathrm{dd}, J 8.7$ and $2.4 \mathrm{~Hz}, 1 \mathrm{H}), 3.87(\mathrm{~s}, 3 \mathrm{H}), 3.02(\mathrm{t}, J 7.5 \mathrm{~Hz}, 2 \mathrm{H}), 2.81(\mathrm{t}$, $J 7.5 \mathrm{~Hz}, 2 \mathrm{H}), 2.42(\mathrm{q}, J 7.2 \mathrm{~Hz}, 2 \mathrm{H}), 1.05$ (t, $J 7.2 \mathrm{~Hz}$, $3 \mathrm{H}) ;{ }^{13} \mathrm{C}$ NMR $\left(75 \mathrm{MHz}, \mathrm{CDCl}_{3}\right) \delta 211.5,153.9,131.4$, $127.6,122.2,115.1,112.2,111.8,100.6,56.0,42.6,36.1$, 19.4, 7.8; IR $v_{\max } / \mathrm{cm}^{-1}(\mathrm{KBr}) 3340(\mathrm{NH}), 1704(\mathrm{C}=\mathrm{O})$, 1487, $1456,1211,1173,806$. HRMS(ESI $\left.{ }^{+}, m / \mathrm{Na}^{+}\right)$Found: 254.11510. Calc. for $\mathrm{C}_{14} \mathrm{H}_{17} \mathrm{O}_{2} \mathrm{~N} / \mathrm{Na}^{+}: 254.11515$.

4-(5-Methoxy-1H-indol-3-yl)-nonan-2-one $(\mathbf{3} \boldsymbol{h}):{ }^{60}$ $90 \%$, viscous brown oil; ${ }^{1} \mathrm{H}$ NMR ( $\left.300 \mathrm{MHz}, \mathrm{CDCl}_{3}\right) \delta 8.35$ (br s, 1H), $7.16(\mathrm{~d}, J 8.7 \mathrm{~Hz}, 1 \mathrm{H}), 7.08(\mathrm{~d}, J 2.4 \mathrm{~Hz}, 1 \mathrm{H}), 6.87$ $(\mathrm{d}, J 2.4 \mathrm{~Hz}, 1 \mathrm{H}), 6.82(\mathrm{dd}, J 8.7$ and $2.4 \mathrm{~Hz}, 1 \mathrm{H}), 3.85(\mathrm{~s}$, $3 \mathrm{H}), 3.42(\mathrm{~m}, 1 \mathrm{H}), 2.85(\mathrm{dd}, J 15.9$ and $7.5 \mathrm{~Hz}, 1 \mathrm{H}), 2.76$ (dd, $J 15.9$ and $6.9 \mathrm{~Hz}, 1 \mathrm{H}), 2.00(\mathrm{~s}, 3 \mathrm{H}), 1.60-1.80(\mathrm{~m}, 2 \mathrm{H})$, 1.38-1,07 (br s, 6H), 0.81 (br s, 3H); ${ }^{13} \mathrm{C}$ NMR $(75 \mathrm{MHz}$, $\left.\mathrm{CDCl}_{3}\right) \delta 209.3,153.4,131.7,126.8,122.0,118.3,111.9$, 111.5, 101.2, 55.8, 50.0, 35.7, 32.6, 31.7, 30.3, 27.1, 22.4, 13.9; IR $v_{\max } / \mathrm{cm}^{-1}$ (neat, film) $3408(\mathrm{NH}), 2954,2929,1707$ $(\mathrm{C}=\mathrm{O}), 1484,1457,1215$.

3-(5-Methoxy-1H-indol-3-yl)-cyclopentanone (3i): viscous brown oil, 95\%; ${ }^{1} \mathrm{H}$ NMR $\left(300 \mathrm{MHz}, \mathrm{CDCl}_{3}\right) \delta$ 8.45 (br s, 1H), $7.21(\mathrm{~d}, J 8.7 \mathrm{~Hz}, 1 \mathrm{H}), 7.05(\mathrm{~d}, J 2.4 \mathrm{~Hz}$, $1 \mathrm{H}), 6.88(\mathrm{~d}, J 2.1 \mathrm{~Hz}, 1 \mathrm{H}), 6.87(\mathrm{dd}, J 8.7$ and $2.1 \mathrm{~Hz}, 1 \mathrm{H})$, $3.85(\mathrm{~s}, 3 \mathrm{H}), 3.62(\mathrm{~m}, 1 \mathrm{H}), 2.72(\mathrm{dd}, J 18.0$ and $7.2 \mathrm{~Hz}, 1 \mathrm{H})$, 2.55-2.22 (m, 4H), 2.15-1.98 (m, 1H); ${ }^{13} \mathrm{C}$ NMR $(75 \mathrm{MHz}$, $\left.\mathrm{CDCl}_{3}\right) \delta 219.8,153.5,131.7,126.8,120.8,117.7,112.0$, $111.9,100.9,55.8,45.1,37.9,33.4,29.5 ; \mathrm{IR} v_{\max } / \mathrm{cm}^{-1}$ (neat, film) $3408(\mathrm{NH}), 1736(\mathrm{C}=\mathrm{O}), 1485,1457,1440$, $1212,1167$.

3-(5-Methoxy-1H-indol-3-yl)-cyclohexanone $(3 \mathbf{j})::^{58}$ $41 \%$, viscous brown oil,; ${ }^{1} \mathrm{H}$ NMR $\left(300 \mathrm{MHz}, \mathrm{CDCl}_{3}\right.$ ) $\delta 8.11(\mathrm{br} \mathrm{s}, 1 \mathrm{H}), 7.24(\mathrm{~d}, J 8.7,1 \mathrm{H}), 7.03(\mathrm{~d}, J 2.4 \mathrm{~Hz}, 1 \mathrm{H})$, $6.95(\mathrm{~d}, J 2.1 \mathrm{~Hz}, 1 \mathrm{H}), 6.94(\mathrm{dd}, J 8.7$ and $2.4 \mathrm{~Hz}, 1 \mathrm{H})$, $3.84(\mathrm{~s}, 3 \mathrm{H}), 3.39(\mathrm{~m}, 1 \mathrm{H}), 2.86-2.73(\mathrm{~m}, 1 \mathrm{H}), 2.63-2.51$ $(\mathrm{m}, 1 \mathrm{H}), 2.51-2.33(\mathrm{~m}, 2 \mathrm{H}), 2.33-2.20(\mathrm{~m}, 1 \mathrm{H}), 2.13-1.73$ $(\mathrm{m}, 3 \mathrm{H}) ;{ }^{13} \mathrm{C} \mathrm{NMR}\left(75 \mathrm{MHz}, \mathrm{CDCl}_{3}\right) \delta 212.0,153.8,131.6$, 126.4, 121.2, 119.2, 112.2, 112.0, 110.9, 56.0, 48.0, 41.5, 35.8, 31.5, 24.9; IR v $v_{\max } / \mathrm{cm}^{-1}$ (neat, film) $3406(\mathrm{NH}), 2360$, $1701(\mathrm{C}=\mathrm{O}), 1484,1456,1216,1171$.

5-(2-Methyl-1H-indol-3-yl)-hexan-3-one (3k): 88\%, viscous brown oil; ${ }^{1} \mathrm{H}$ NMR $\left(300 \mathrm{MHz}, \mathrm{CDCl}_{3}\right) \delta 7.96$ (br. s,
1H), $7.59(\mathrm{~m}, 1 \mathrm{H}), 7.17-7.10(\mathrm{~m}, 1 \mathrm{H}), 7.09-6.98(\mathrm{~m}, 2 \mathrm{H})$, $3.55(\mathrm{~m}, 1 \mathrm{H}), 2.97(\mathrm{dd}, J 15.6$ and $7.8 \mathrm{~Hz}), 2.76(\mathrm{dd}, J 15.6$ and 7.2 Hz), 2.32-2.21 (m, 5H), $1.39(\mathrm{~d}, J 7.2 \mathrm{~Hz}, 3 \mathrm{H}), 0.87$ (d, $J 7.5 \mathrm{~Hz}, 3 \mathrm{H}) ;{ }^{13} \mathrm{C}$ NMR $\left(75 \mathrm{MHz}, \mathrm{CDCl}_{3}\right) \delta 211.8,135.4$, 130.4, 126.8, 120.3, 118.7, 118.6, 114.6, 110.5, 49.1, 36.5, 27.1, 21.0, 11.6, 7.5; IR $v_{\max } / \mathrm{cm}^{-1}$ (neat, film) $3400(\mathrm{NH})$, 2969, 2933, $1705(\mathrm{C}=\mathrm{O}), 1461,742$. HRMS $\left(\mathrm{ESI}^{+}, m / \mathrm{Na}^{+}\right)$ Found: 252.13575. Calc. for $\mathrm{C}_{15} \mathrm{H}_{19} \mathrm{ON} / \mathrm{Na}^{+}: 252.13589$.

1-(2-Methyl-1H-indol-3-yl)pentan-3-one (3l): 97\%, viscous brown oil; ${ }^{1} \mathrm{H}$ NMR $\left(300 \mathrm{MHz}, \mathrm{CDCl}_{3}\right) \delta 7.91$ (br $\mathrm{s}, 1 \mathrm{H}), 7.44(\mathrm{~m}, 1 \mathrm{H}), 7.20-6.94(\mathrm{~m}, 3 \mathrm{H}), 2.95(\mathrm{t}, J 7.2 \mathrm{~Hz}$, 2H), $2.68(\mathrm{t}, J 7.2 \mathrm{~Hz}, 2 \mathrm{H}), 2.35-2.20(\mathrm{~m}, 5 \mathrm{H}), 0.97(\mathrm{t}$, $J 7.2 \mathrm{~Hz}, 3 \mathrm{H}) ;{ }^{13} \mathrm{C}$ NMR $\left(75 \mathrm{MHz}, \mathrm{CDCl}_{3}\right) \delta 211.9,135.1$, 131.1, 128.0, 120.6, 118.8, 117.5, 110.3, 110.0, 42.7, 36.1, 18.4, 11.2, 7.6; IR $v_{\text {max }} / \mathrm{cm}^{-1}$ (neat, film) $3400(\mathrm{NH}), 2975$, 2936, 2919, $1708(\mathrm{C}=\mathrm{O}), 1462,742$. HRMS $\left(\mathrm{ESI}^{+}, m / \mathrm{Na}^{+}\right)$ Found: 238.12015. Calc. for $\mathrm{C}_{14} \mathrm{H}_{17} \mathrm{ON} / \mathrm{Na}^{+}$: 238.12024.

3-(2-Methyl-1H-indol-3-yl)-cyclopentanone $(3 \boldsymbol{m}): 46$ $81 \%$, yellow solid; mp $135-136{ }^{\circ} \mathrm{C}$; ${ }^{1} \mathrm{H}$ NMR $(300 \mathrm{MHz}$, $\left.\mathrm{CDCl}_{3}\right) \delta 7.98($ br s, $1 \mathrm{H}), 7.53(\mathrm{~d}, J 7.5 \mathrm{~Hz}, 1 \mathrm{H}), 7.28(\mathrm{~d}$, $J 7.8 \mathrm{~Hz}, 1 \mathrm{H}), 7.23-7.00(\mathrm{~m}, 2 \mathrm{H}), 3.59(\mathrm{~m}, 1 \mathrm{H}), 2.87-2.20$ $(\mathrm{m}, 10 \mathrm{H}) ;{ }^{13} \mathrm{C}$ NMR $\left(75 \mathrm{MHz}, \mathrm{CDCl}_{3}\right) \delta 219.8,135.5$, 130.9, 126.7, 120.9, 119.1, 118.6, 111.8, 110.7, 44.3, 39.4, $34.5,29.7,12.1 ; \mathrm{IR} v_{\max } / \mathrm{cm}^{-1}(\mathrm{KBr}) 3342(\mathrm{NH}), 2961,2897$, $1729(\mathrm{C}=\mathrm{O}), 1461,1231,1137,743$.

General procedure for the synthesis of $\beta$-indolylchalcones $3 r-z$

In a $25 \mathrm{~mL}$ round-bottom flask, equipped with a reflux condenser and magnetic stirrer were added the indoles $1 \mathbf{a}-\mathbf{c}(1.0 \mathrm{mmol})$, the chalcones $2 \mathbf{f}-\mathbf{i}(1.1 \mathrm{mmol})$, and $\mathrm{SnCl}_{2} \cdot 2 \mathrm{H}_{2} \mathrm{O}(0.1 \mathrm{mmol})$ in $i$-PrOH $(2 \mathrm{~mL})$. The reaction mixture was heated to reflux and stirred for the appropriate time showed in the Table 3. After the completion of the reaction, monitored by TLC, the solvent was removed under vaccum and the crude products $\mathbf{3 r}$-z were purified by column chromatography using hexanes/ethyl acetate as eluant.

3-(1H-Indol-3-yl)-1,3-diphenylpropan-1-one (3r): ${ }^{64}$ 96\%,brownish solid, mp 130-131 ${ }^{\circ} \mathrm{C} ;{ }^{1} \mathrm{H}$ NMR $(300 \mathrm{MHz}$, $\left.\mathrm{CDCl}_{3}\right) \delta 8.03$ (br s, 1H), $7.91(\mathrm{~d}, J 7.5 \mathrm{~Hz}, 2 \mathrm{H}), 7.51$ (t, $J 7.2 \mathrm{~Hz}, 1 \mathrm{H}), 7.46-7.06(\mathrm{~m}, 10 \mathrm{H}), 6.99(\mathrm{t}, J 7.2 \mathrm{~Hz}, 1 \mathrm{H})$, $6.90(\mathrm{~s}, 1 \mathrm{H}), 5.05(\mathrm{~m}, 1 \mathrm{H}), 3.80(\mathrm{dd}, J 16.8$ and $6.9 \mathrm{~Hz}$, $1 \mathrm{H}), 3.70(\mathrm{dd}, J 16.8$ and $7.8 \mathrm{~Hz}, 1 \mathrm{H}) ;{ }^{13} \mathrm{C}$ NMR $(75 \mathrm{MHz}$, $\left.\mathrm{CDCl}_{3},\right) \delta 198.7,144.2,137.0,136.6,133.0,128.5,128.4$, $128.1,127.8,126.5,126.2,122.0,121.4,119.4,119.3$, 119.1, 111.1, 45.2, 38.2; IR $v_{\max } / \mathrm{cm}^{-1}(\mathrm{KBr}) 3411(\mathrm{NH})$, $1681(\mathrm{C}=\mathrm{O}), 1456,1448,745,700$. 
3-(1H-Indol-3-yl)-3-(naphthalen-1-yl)-1-phenylpropan-1-one (3s): $60 \%$, brownish solid, mp $97-98^{\circ} \mathrm{C}$; ${ }^{1} \mathrm{H}$ NMR $\left(300 \mathrm{MHz}, \mathrm{CDCl}_{3}\right) \delta 8.29(\mathrm{~m}, 1 \mathrm{H}), 7.96-$ $7.80(\mathrm{~m}, 4 \mathrm{H}), 7.68(\mathrm{~d}, J 7.5 \mathrm{~Hz}, 1 \mathrm{H}), 7.54-7.17(\mathrm{~m}$, 9H), $7.09(\mathrm{~m}, 1 \mathrm{H}), 6.96(\mathrm{~m}, 1 \mathrm{H}), 6.70(\mathrm{~s}, 1 \mathrm{H}), 5.92$ $(\mathrm{m}, 1 \mathrm{H}), 3.94(\mathrm{dd}, J 17.4$ and $7.8 \mathrm{~Hz}, 1 \mathrm{H}), 3.73(\mathrm{dd}$, $J 17.4$ and $6.3 \mathrm{~Hz}, 1 \mathrm{H}) ;{ }^{13} \mathrm{C}$ NMR $\left(75 \mathrm{MHz}, \mathrm{CDCl}_{3}\right.$,) $\delta$ 198.5, 139.9, 137.0, 136.6, 134.0, 133.1, 131.4, 128.8, $128.6,128.0,127.0,126.6,126.1,125.5,125.3,124.4$, $123.6,122.2,122.0,119.4,119.3,118.9,111.2,44.6$, 33.2; IR $v_{\max } / \mathrm{cm}^{-1}(\mathrm{KBr}) 3412(\mathrm{NH}), 3055,1681(\mathrm{C}=\mathrm{O})$, 1596, 1448, 1337, 1269, 780, 743; HRMS (ESI $\left.{ }^{+}, m / \mathrm{Na}^{+}\right)$ Found: 398.15161.Calc. for $\mathrm{C}_{27} \mathrm{H}_{21} \mathrm{ON} / \mathrm{Na}^{+}$: 398.15154 .

3-(1H-Indol-3-yl)-3-(4-methoxyphenyl)-1-phenylpropan-1-one $(3 t):{ }^{64} 80 \%$, brownish solid, $\mathrm{mp} 114-115^{\circ} \mathrm{C}$; ${ }^{1} \mathrm{H}$ NMR $\left(300 \mathrm{MHz}, \mathrm{CDCl}_{3}\right) \delta 8.00($ br s, $1 \mathrm{H}), 7.92$ $(\mathrm{d}, J 7.8 \mathrm{~Hz}, 2 \mathrm{H}), 7.53(\mathrm{t}, J 7.5 \mathrm{~Hz}, 1 \mathrm{H}), 7.47-7.36(\mathrm{~m}$, $3 \mathrm{H}), 7.29(\mathrm{~d}, J 7.8 \mathrm{~Hz}, 1 \mathrm{H}), 7.25(\mathrm{~d}, J 8.4 \mathrm{~Hz}, 2 \mathrm{H})$, $7.13(\mathrm{t}, J 7.5 \mathrm{~Hz}, 1 \mathrm{H}), 7.00(\mathrm{t}, J 7.5,1 \mathrm{H}), 6.95(\mathrm{~d}$, $J 1.5 \mathrm{~Hz}, 1 \mathrm{H}), 6.78(\mathrm{~d}, J 8.4 \mathrm{~Hz}, 2 \mathrm{H}), 5.01(\mathrm{t}, J 6.9 \mathrm{~Hz}, 1 \mathrm{H})$, 3.83-3.63 (m, 5H); $\delta{ }^{13} \mathrm{C}$ NMR (75 MHz, $\mathrm{CDCl}_{3}$, ) 198.8, 157.9, 137.1, 136.6, 136.3, 133.0, 128.7, 128.5, 128.1, 126.5, 122.0, 121.3, 119.5, 119.3, 113.7, 111.1, 55.1, 45.3, 37.4; IR $v_{\text {max }} / \mathrm{cm}^{-1}(\mathrm{KBr}) 3420(\mathrm{NH}), 1670(\mathrm{C}=\mathrm{O}), 1510$, 1249, 1177, 1031, 746.

3-(1H-Indol-3-yl)-1-phenyl-3-(thiophen-2-yl)-propan1-one $(3 \boldsymbol{u}):{ }^{65} 74 \%$, brown solid, mp 153-154 ${ }^{\circ} \mathrm{C} ;{ }^{1} \mathrm{H}$ NMR $\left(300 \mathrm{MHz}, \mathrm{CDCl}_{3}\right) \delta 8.02($ br s, $1 \mathrm{H}), 7.93(\mathrm{~d}, J 8.1 \mathrm{~Hz}$, $2 \mathrm{H}), 7.53(\mathrm{t}, J 7.5 \mathrm{~Hz}, 2 \mathrm{H}), 7.42(\mathrm{t}, J 7.5 \mathrm{~Hz}, 2 \mathrm{H}), 7.31(\mathrm{~d}$, $J$ 8.1 Hz, 1H), 7.20-7.00 (m, 4H), 6.95-6.83 (m, 2H), 5.36 $(\mathrm{t}, J 7.2 \mathrm{~Hz}, 1 \mathrm{H}), 3.83(\mathrm{~d}, J 7.2 \mathrm{~Hz}, 2 \mathrm{H}) ;{ }^{13} \mathrm{C} \mathrm{NMR}(75 \mathrm{MHz}$, $\left.\mathrm{CDCl}_{3},\right) \delta 198.1,148.7,137.0,136.5,133.1,128.6,128.1$, $126.5,126.2,124.2,123.4,122.2,121.6,119.5,119.4$, 119.0, 111.2, 46.1, 33.5; IR $v_{\max } / \mathrm{cm}^{-1}(\mathrm{KBr}) 3409(\mathrm{NH})$, 3057, $1682(\mathrm{C}=\mathrm{O}), 1268,743,689$.

3-(5-Methoxy-1H-indol-3-yl)-1,3-diphenylpropan-1one (3v): ${ }^{66} 96 \%$, yellow solid, mp $136-137{ }^{\circ} \mathrm{C}$; ${ }^{1} \mathrm{H}$ NMR $\left(300 \mathrm{MHz}, \mathrm{CDCl}_{3}\right) \delta 8.02($ br s, $1 \mathrm{H}), 7.92(\mathrm{~d}, J 7.2 \mathrm{~Hz}$, $2 \mathrm{H}), 7.6-7.1(\mathrm{~m}, 9 \mathrm{H}), 6.92(\mathrm{~d}, J 1.5 \mathrm{~Hz}, 1 \mathrm{H}), 6.85-6.73(\mathrm{~m}$, $2 \mathrm{H}), 5.00(\mathrm{~m}, 1 \mathrm{H}), 3.84-3.64(\mathrm{~m}, 5 \mathrm{H}) ;{ }^{13} \mathrm{C}$ NMR $(75 \mathrm{MHz}$, $\left.\mathrm{CDCl}_{3}\right) \delta 198.7,153.7,144.1,137.1,133.0,131.7,128.5$, 128.4, 128.1, 127.8, 127.0, 126.2, 122.2, 118.8, 112.1, 111.8, 101.4, 55.7, 45.1, 38.2; IR $v_{\max } / \mathrm{cm}^{-1}(\mathrm{KBr}) 3366$ $(\mathrm{NH}), 1679(\mathrm{C}=\mathrm{O})$, 1485, 1457, 1448, 1214, 1170, 723.

3-(5-Methoxy-1H-indol-3-yl)-3-(naphthalen-1-yl)-1phenylpropan-1-one $(3 w)$ : 95\%, pale brown solid, mp 156$157^{\circ} \mathrm{C} ;{ }^{1} \mathrm{H}$ NMR $\left(300 \mathrm{MHz}, \mathrm{CDCl}_{3}\right) \delta 8.35-8.26(\mathrm{~m}, 1 \mathrm{H})$, $7.94(\mathrm{~d}, J 7.8 \mathrm{~Hz}, 2 \mathrm{H}), 7.90-7.78(\mathrm{~m}, 2 \mathrm{H}), 7.70(\mathrm{~d}, J 6.9 \mathrm{~Hz}$, $1 \mathrm{H}), 7.58-7.29(\mathrm{~m}, 7 \mathrm{H}), 7.15(\mathrm{~d}, J 9.0 \mathrm{~Hz}, 1 \mathrm{H}), 6.85(\mathrm{~d}$, $J 2.1 \mathrm{~Hz}, 1 \mathrm{H}), 6.82-6.74(\mathrm{~m}, 2 \mathrm{H}), 5.88(\mathrm{~m}, 1 \mathrm{H}), 3.95(\mathrm{dd}$, $J 17.4$ and $7.8 \mathrm{~Hz}, 1 \mathrm{H}), 3.79$ (dd, $J 17.4$ and $6.3 \mathrm{~Hz}, 1 \mathrm{H}$ ), $3.66(\mathrm{~s}, 3 \mathrm{H}) ;{ }^{13} \mathrm{C}$ NMR $\left(75 \mathrm{MHz}, \mathrm{CDCl}_{3}\right) \delta 198.5,153.8$, $139.8,137.1,134.1,133.1,131.8,131.5,128.6,128.5$, $128.1,127.1,127.0,126.1,125.5,125.3,124.3,123.6$, $122.9,118.8,112.2,111.8,101.3 ; 55.8,44.6,33.2$; IR $v_{\max } / \mathrm{cm}^{-1}(\mathrm{KBr}) 3412(\mathrm{NH}), 1680(\mathrm{C}=\mathrm{O}), 1484,1212$, 780; HRMS $\left(\mathrm{ESI}^{+}, m / \mathrm{Na}^{+}\right)$Found: 428.16208. Calc. for $\mathrm{C}_{28} \mathrm{H}_{23} \mathrm{O}_{2} \mathrm{~N} / \mathrm{Na}^{+}$: 428.16210 .

3-(5-Methoxy-1H-indol-3-yl)-3-(4-methoxyphenyl)-1phenylpropan-1-one $(\mathbf{3 x})$ : $95 \%$, brown solid, $\mathrm{mp} 70-71^{\circ} \mathrm{C}$; ${ }^{1} \mathrm{H}$ NMR $\left(300 \mathrm{MHz}, \mathrm{CDCl}_{3}\right) \delta 8.02(\mathrm{br} \mathrm{s}, 1 \mathrm{H}), 7.91(\mathrm{~d}$, $J 7.8 \mathrm{~Hz}, 2 \mathrm{H}), 7.52(\mathrm{t}, J 7.8 \mathrm{~Hz}, 1 \mathrm{H}), 7.40(\mathrm{t}, J 7.5 \mathrm{~Hz}$, $2 \mathrm{H}), 7.24(\mathrm{~d}, J 8.7 \mathrm{~Hz}, 2 \mathrm{H}), 7.14(\mathrm{~d}, J 8.7 \mathrm{~Hz}, 1 \mathrm{H}), 6.90(\mathrm{~s}$, $1 \mathrm{H}), 6.83(\mathrm{~d}, J 2.1 \mathrm{~Hz}, 1 \mathrm{H}), 6.77(\mathrm{~d}, J 8.4 \mathrm{~Hz}, 3 \mathrm{H}), 4.95$ (t, $J 7.2 \mathrm{~Hz}, 1 \mathrm{H}), 3.81-3.61(\mathrm{~m}, 8 \mathrm{H}) ;{ }^{13} \mathrm{C}$ NMR $(75 \mathrm{MHz}$, $\left.\mathrm{CDCl}_{3}\right) \delta 198.9,157.9,153.6,137.1,136.2,133.0,131.7$, 128.7, 128.5, 128.0, 126.9, 122.1, 119.1, 113.7, 112.0, $111.8,101.4,55.7,55.1,45.2,37.4 ; \mathrm{IR} v_{\max } / \mathrm{cm}^{-1}(\mathrm{KBr})$ $3400(\mathrm{NH}), 2929,1683(\mathrm{C}=\mathrm{O}), 1509,1485,1247,1212$, 1175, 1033; HRMS (ESI $\left.{ }^{+}, m / \mathrm{Na}^{+}\right)$Found: 408.15689. Calc. for $\mathrm{C}_{25} \mathrm{H}_{23} \mathrm{O}_{3} \mathrm{~N} / \mathrm{Na}^{+}: 408.15702$.

3-(5-Methoxy-1H-indol-3-yl)-1-phenyl-3-(thiophen2-yl)propan-1-one (3y): 92\%, pale yellow solid, mp 153-154 ${ }^{\circ} \mathrm{C}$; ${ }^{1} \mathrm{H}$ NMR $\left(300 \mathrm{MHz}, \mathrm{CDCl}_{3}\right) \delta$ 7.98$7.88(\mathrm{~m}, 3 \mathrm{H}), 7.54(\mathrm{~m}, 1 \mathrm{H}), 7.43(\mathrm{~m}, 2 \mathrm{H}), 7.21(\mathrm{~d}$, $J 8.7 \mathrm{~Hz}, 1 \mathrm{H}), 7.10(\mathrm{~m}, 1 \mathrm{H}), 7.04(\mathrm{~d}, J 2.4 \mathrm{~Hz}, 1 \mathrm{H}), 6.95$ $(\mathrm{d}, J 2.4 \mathrm{~Hz}, 1 \mathrm{H}), 6.94-6.85(\mathrm{~m}, 2 \mathrm{H}), 6.83$ (dd, $J 8.7$ and $2.4 \mathrm{~Hz}, 1 \mathrm{H}), 5.32(\mathrm{~m}, 1 \mathrm{H}), 3.82-3.75(\mathrm{~m}, 5 \mathrm{H}) ;{ }^{13} \mathrm{C} \mathrm{NMR}$ $\left(75 \mathrm{MHz}, \mathrm{CDCl}_{3}\right) \delta 198.1,153.9,148.7,137.0,133.1$, 131.6, 128.6, 128.1, 126.7, 126.5, 124.2, 123.5, 122.3, $118.8,112.3,111.9,101.3,55.9,46.0,33.4 ; \mathrm{IR} v_{\max } / \mathrm{cm}^{-1}$ $(\mathrm{KBr}) 3382(\mathrm{NH}), 1680(\mathrm{C}=\mathrm{O}), 1485,1438,1213,1171$, 732; HRMS (ESI ${ }^{+}, m / \mathrm{Na}^{+}$) Found: 384.10283. Calc. for $\mathrm{C}_{22} \mathrm{H}_{19} \mathrm{O}_{2} \mathrm{~N} / \mathrm{Na}^{+}: 384.10287$.

3-(2-Methyl-1H-indol-3-yl)-1,3-diphenylpropan1-one $(3 z): 4696 \%$, brownish solid, $\mathrm{mp} 130-131{ }^{\circ} \mathrm{C}$; ${ }^{1} \mathrm{H} \mathrm{NMR}\left(300 \mathrm{MHz}, \mathrm{CDCl}_{3}\right) \delta 8.03(\mathrm{br} \mathrm{s}, 1 \mathrm{H}), 7.91(\mathrm{~d}$, $J 7.5 \mathrm{~Hz}, 2 \mathrm{H}), 7.51(\mathrm{t}, J 7.2 \mathrm{~Hz}, 1 \mathrm{H}), 7.46-7.06(\mathrm{~m}, 10 \mathrm{H})$, $6.99(\mathrm{t}, J 7.2 \mathrm{~Hz}, 1 \mathrm{H}), 6.90(\mathrm{~s}, 1 \mathrm{H}), 5.05(\mathrm{~m}, 1 \mathrm{H}), 3.80$ (dd, $J 16.8$ and $6.9 \mathrm{~Hz}, 1 \mathrm{H}$ ), 3.70 (dd, $J 16.8$ and $7.8 \mathrm{~Hz}$, $1 \mathrm{H}) ;{ }^{13} \mathrm{C}$ NMR $\left(75 \mathrm{MHz}, \mathrm{CDCl}_{3}\right) \delta 198.7,144.2,137.0$, 136.6, 133.0, 128.5, 128.4, 128.1, 127.8, 126.5, 126.2, 122.0, 121.4, 119.4, 119.3, 119.1, 111.1, 45.2, 38.2; IR $v_{\max } / \mathrm{cm}^{-1}(\mathrm{KBr}) 3411(\mathrm{NH}), 1681(\mathrm{C}=\mathrm{O}), 1456,1448$, 745,700 . 


\section{Procedure for the synthesis of nitro-adducts 5 a in i-PrOH}

In a $25 \mathrm{~mL}$ round botton flask equipped with a reflux condenser, and magnetic stirrer was added the indole $1 \mathrm{a}(1.0 \mathrm{mmol})$, the nitroolefin $4 \mathrm{a}(1.1 \mathrm{mmol})$, and the $\mathrm{CoCl}_{2} \cdot 6 \mathrm{H}_{2} \mathrm{O}(0.1 \mathrm{mmol})$ in $i$ - $\mathrm{PrOH}(2 \mathrm{~mL})$. The reaction mixture was heated to reflux temperature of $i-\mathrm{PrOH}$ and stirred for $2 \mathrm{~h}$. After the completion of the reaction, monitored by TLC, the solvent was removed under vaccum and the crude product $\mathbf{5} \mathbf{a}$ was purified by column chromatography using a mixture of hexanes/ethyl acetate 70:30 (v/v) as eluent.

3-(2-Nitro-1-phenylethyl)-1H-indole (5a): ${ }^{62} 97 \%$, brownish solid, mp 99-100 ${ }^{\circ} \mathrm{C} ;{ }^{1} \mathrm{H} \mathrm{NMR}\left(300 \mathrm{MHz}, \mathrm{CDCl}_{3}\right)$ $\delta 7.84(\mathrm{br} \mathrm{s}, 1 \mathrm{H}), 7.37(\mathrm{~d}, J 8.1 \mathrm{~Hz}, 1 \mathrm{H}), 7.25-7.05(\mathrm{~m}, 7 \mathrm{H})$, $7.00(\mathrm{t}, J 7.2 \mathrm{~Hz}, 1 \mathrm{H}), 6.72(\mathrm{~d}, J 1.8 \mathrm{~Hz}, 1 \mathrm{H}), 5.08(\mathrm{~m}, 1 \mathrm{H})$, $4.87(\mathrm{dd}, J 12.3$ and $7.5 \mathrm{~Hz}, 1 \mathrm{H}), 4.76(\mathrm{dd}, J 12.3$ and 8.4 $\mathrm{Hz}, 1 \mathrm{H}) ;{ }^{13} \mathrm{C} \mathrm{NMR}\left(75 \mathrm{MHz}, \mathrm{CDCl}_{3}\right) \delta 139.1,136.2,128.7$, $127.6,127.3,125.8,122.3,121.5,119.6,118.6,113.7$, $111.3,79.3,41.3 ; \mathrm{IR} v_{\max } / \mathrm{cm}^{-1}(\mathrm{KBr}) 3401(\mathrm{NH}), 3052$, 1536, 1455, 1424, 1377, 1097, 750, 704.

\section{General procedure for the synthesis of nitro-adducts $\mathbf{5} \boldsymbol{b}-\boldsymbol{q}$} under solventless conditions

In a $25 \mathrm{~mL}$ round-botton flask equiped with a reflux condenser, and magnetic stirrer, were added the indoles 1a-c $(1.0 \mathrm{mmol})$, the nitroolefins $4 \mathbf{b}-\mathbf{g}(1.1 \mathrm{mmol})$ and $\mathrm{CoCl}_{2} \cdot 6 \mathrm{H}_{2} \mathrm{O}(0.1 \mathrm{mmol})$. The reactional flask was placed in a $100{ }^{\circ} \mathrm{C}$ pre-heated silicone oil bath and was stirred for the appropriate time showed in the Table 6. After the completion of the reaction, monitored by TLC, the crude product $\mathbf{5 b}-\mathbf{q}$ were purified by column chromatography using a mixture of hexanes/ethyl acetate.

3-(1-(4-Methoxyphenyl)-2-nitroethyl)-1H-indole (5b):67 92\%, brownish solid, mp $146-147{ }^{\circ} \mathrm{C}$; ${ }^{1} \mathrm{H}$ NMR $(300 \mathrm{MHz}$, $\left.\mathrm{CDCl}_{3}\right) \delta 8.10(\mathrm{br} \mathrm{s}, 1 \mathrm{H}), 7.43(\mathrm{~d}, J 8.1 \mathrm{~Hz}, 1 \mathrm{H}), 7.35(\mathrm{~d}$, $J 8.1 \mathrm{~Hz}, 1 \mathrm{H}), 7.24(\mathrm{~d}, J 8.4 \mathrm{~Hz}, 2 \mathrm{H}), 7.19(\mathrm{t}, J 7.5 \mathrm{~Hz}$, $1 \mathrm{H}), 7.07(\mathrm{t}, J 7.5 \mathrm{~Hz}, 1 \mathrm{H}), 7.01(\mathrm{~d}, J 1.5 \mathrm{~Hz}, 1 \mathrm{H}), 6.84(\mathrm{~d}$, $J 8.4 \mathrm{~Hz}, 2 \mathrm{H}), 5.14(\mathrm{~m}, 1 \mathrm{H}), 5.04(\mathrm{dd}, J 12.3$ and $7.5 \mathrm{~Hz}$, $1 \mathrm{H}), 4.89(\mathrm{dd}, J 12.3$ and $8.4 \mathrm{~Hz}, 1 \mathrm{H}), 3.77$ (s, $3 \mathrm{H})$; ${ }^{13} \mathrm{C} \mathrm{NMR}\left(75 \mathrm{MHz}, \mathrm{CDCl}_{3}\right) \delta 158.8,136.5,131.2,128.8$, 126.1, 122.6, 121.4, 119.9, 119.0, 114.8, 114.3, 111.3, 79.7, 55.2, 40.8; IR $v_{\max } / \mathrm{cm}^{-1}(\mathrm{KBr}) 3379(\mathrm{NH}), 1547,1511$, $1463,1422,1377,1245,1029,750$.

3-(1-(4-Fluorophenyl)-2-nitroethyl)-1H-indole (5c): ${ }^{67}$ $97 \%$, orange solid, mp 77-78 ${ }^{\circ} \mathrm{C}$; ${ }^{1} \mathrm{H}$ NMR $(300 \mathrm{MHz}$, $\left.\mathrm{CDCl}_{3}\right) \delta 8.10($ br s, $1 \mathrm{H}), 7.39(\mathrm{~d}, J 7.8 \mathrm{~Hz}, 1 \mathrm{H}), 7.37-$ $7.16(\mathrm{~m}, 4 \mathrm{H}), 7.11-6.95(\mathrm{~m}, 4 \mathrm{H}), 5.16(\mathrm{~m}, 1 \mathrm{H}), 5.04(\mathrm{dd}$,
$J 12.6$ and $7.5 \mathrm{~Hz}, 1 \mathrm{H}), 4.89$ (dd, $J 12.6$ and $8.7 \mathrm{~Hz}, 1 \mathrm{H})$; ${ }^{13} \mathrm{C}$ NMR $\left(75 \mathrm{MHz}, \mathrm{CDCl}_{3}\right) \delta 162.1(\mathrm{~d}, J 245 \mathrm{~Hz}), 136.5$, 134.9 (d, J $3 \mathrm{~Hz}), 129.3$ (d, J 8 Hz), 125.9, 122.8, 121.4, 120.0, 118.8, $115.8(\mathrm{~d}, J 21 \mathrm{~Hz}), 114.2,111.4,79.5,40.8$; IR $v_{\max } / \mathrm{cm}^{-1}(\mathrm{KBr}) 3371(\mathrm{NH}), 1542,1508,1430,1378$, 1224,748 .

$4-(1-(1 H-I n d o l-3-y l)-2-$ nitroethyl $)-N, N-$ dimethylaniline $(\mathbf{5 d}):{ }^{68} 98 \%$, pink solid, $\mathrm{mp} 131-133{ }^{\circ} \mathrm{C}$; ${ }^{1} \mathrm{H} \mathrm{NMR}\left(300 \mathrm{MHz}, \mathrm{CDCl}_{3}\right) \delta 8.09$ (br s, $\left.1 \mathrm{H}\right) ; 7.46(\mathrm{~d}$, $J 8.1 \mathrm{~Hz}, 1 \mathrm{H}) ; 7.35(\mathrm{~d}, J 8.1 \mathrm{~Hz}, 1 \mathrm{H}) ; 7.24-7.00(\mathrm{~m}, 5 \mathrm{H})$; $6.71(\mathrm{~d}, J 8.4 \mathrm{~Hz}, 2 \mathrm{H}) ; 5.10(\mathrm{~m}, 1 \mathrm{H}) ; 5.03(\mathrm{dd}, J 11.7$ and $7.5 \mathrm{~Hz}, 1 \mathrm{H}) ; 4.88$ (dd, $J 11.7$ and $8.4 \mathrm{~Hz}, 1 \mathrm{H}) ; 2.92$ (s, $6 \mathrm{H}) ;{ }^{13} \mathrm{C}$ NMR $\left(75 \mathrm{MHz}, \mathrm{CDCl}_{3}\right) \delta 149.8 ; 136.5 ; 128.4$; $126.7 ; 126.2 ; 122.5 ; 121.4 ; 119.8 ; 119.1 ; 115.1 ; 112.7$; $111.3 ; 79.9 ; 40.8 ; 40.5 ; \mathrm{IR} v_{\max } / \mathrm{cm}^{-1}(\mathrm{KBr}) 3434(\mathrm{NH})$, 2916, 1611, 1519, 1379, 817, 743.

3-(1-(3,4-Dimethoxyphenyl)-2-nitroethyl)-1H-indole (5e): ${ }^{69} 90 \%$, orange solid, mp 79-80 ${ }^{\circ} \mathrm{C} ;{ }^{1} \mathrm{H}$ NMR (300 MHz, CDCl3) $\delta 8.27$ (br s, $1 \mathrm{H}), 7.43$ (d, $J 7.8 \mathrm{~Hz}$, 1H), $7.28(\mathrm{~d}, J 8.1 \mathrm{~Hz}, 1 \mathrm{H}), 7.16(\mathrm{t}, J 7.5 \mathrm{~Hz}, 1 \mathrm{H}), 7.05$ (t, J 7.5 Hz, 1H), $6.93(\mathrm{~s}, 1 \mathrm{H}), 6.87-6.80(\mathrm{~m}, 2 \mathrm{H}), 6.76(\mathrm{~d}$, $J 8.1 \mathrm{~Hz}, 1 \mathrm{H}), 5.11(\mathrm{~m}, 1 \mathrm{H}), 5.00(\mathrm{dd}, J 12.3$ and $7.5 \mathrm{~Hz}$, $1 \mathrm{H}), 4.87(\mathrm{dd}, J 12.3$ and $8.4 \mathrm{~Hz}, 1 \mathrm{H}), 3.79$ (s, 3H), 3.76 (s, 3H); ${ }^{13} \mathrm{C}$ NMR (75 MHz, CDCl3) $\delta$ 149.0, 148.2, 136.4, 131.7, 125.9, 122.4, 121.5, 119.7, 119.6, 118.7, 114.2, 111.4, 111.3, 111.1, 79.6, 55.7, 41.1; IR $v_{\max } / \mathrm{cm}^{-1}(\mathrm{KBr})$ $3405(\mathrm{NH}), 1549,1515,1262,1141,1023,745$.

3-(1-(Naphthalen-1-yl)-2-nitroethyl)-1H-indole (5f) ${ }^{67}$ $97 \%$, redish solid, mp 140-142 ${ }^{\circ} \mathrm{C} ;{ }^{1} \mathrm{H}$ NMR $(300 \mathrm{MHz}$, $\left.\mathrm{CDCl}_{3}\right) \delta 8.27$ (d, J 8.4 Hz), 8.05 (br s, $\left.1 \mathrm{H}, \mathrm{NH}\right), 7.91$ $7.86(\mathrm{~m}, 1 \mathrm{H}), 7.82-7.74(\mathrm{~m}, 1 \mathrm{H}), 7.59-7.32(\mathrm{~m}, 6 \mathrm{H}), 7.23-$ $7.15(\mathrm{~m}, 1 \mathrm{H}), 7.09-6.97(\mathrm{~m}, 2 \mathrm{H}), 6.07(\mathrm{~m}, 1 \mathrm{H}), 5.12(\mathrm{dd}$, $J 12.9$ and $8.1 \mathrm{~Hz}, 1 \mathrm{H}), 5.07$ (dd, $J 12.9$ and $7.2 \mathrm{~Hz}, 1 \mathrm{H}$ ); ${ }^{13} \mathrm{C} \mathrm{NMR}\left(75 \mathrm{MHz}, \mathrm{CDCl}_{3}\right) \delta 136.6,134.6,134.2,131.1$, 129.1, 128.3, 126.8, 126.1, 125.9, 125.3, 124.6, 122.7, 122.6, 120.0, 118.8, 114.3, 111.4, 78.5, 37.0; IR $v_{\max } / \mathrm{cm}^{-1}$ (KBr) 3420 (NH), 1545, 1509, 1458, 1381, 796, 777, 743.

3-(2-Nitro-1-(thiophen-2-yl)ethyl)-1H-indole (5g): ${ }^{69}$ 98\%, brown solid, mp 86-87 ${ }^{\circ} \mathrm{C}$; ${ }^{1} \mathrm{H}$ NMR $(300 \mathrm{MHz}$, $\left.\mathrm{CDCl}_{3}\right) \delta 8.03($ br s, $1 \mathrm{H}), 7.49(\mathrm{~d}, J 8.1 \mathrm{~Hz}, 1 \mathrm{H}), 7.29$ (d, $J 8.1 \mathrm{~Hz}, 1 \mathrm{H}), 7.22-7.05(\mathrm{~m}, 3 \mathrm{H}), 6.98(\mathrm{~d}, J 2.7 \mathrm{~Hz}$, $1 \mathrm{H}), 6.96-6.87(\mathrm{~m}, 2 \mathrm{H}), 5.42(\mathrm{~m}, J 7.8 \mathrm{~Hz}, 1 \mathrm{H}), 4.99$ (dd, $J 12.3$ and $8.1 \mathrm{~Hz}, 1 \mathrm{H}), 4.92(\mathrm{dd}, J 12.3$ and $8.1 \mathrm{~Hz}, 1 \mathrm{H})$; ${ }^{13} \mathrm{C}$ NMR $\left(75 \mathrm{MHz}, \mathrm{CDCl}_{3}\right) \delta 142.8,136.2,126.9,125.5$, $125.1,124.8,122.5,121.9,119.9,118.6,113.6,111.5$, 79.8, 36.7; IR $v_{\max } / \mathrm{cm}^{-1}(\mathrm{KBr}) 3416(\mathrm{NH}), 1550,1455$, $1433,1418,1377,747,720$. 
5-Methoxy-3-(2-nitro-1-phenylethyl)-1H-indole (5h):67 $95 \%$, brownish solid, mp $130-131{ }^{\circ} \mathrm{C} ;{ }^{1} \mathrm{H}$ NMR $(300 \mathrm{MHz}$, $\left.\mathrm{CDCl}_{3}\right) \delta 8.02(\mathrm{br} \mathrm{s}, 1 \mathrm{H}), 7.32-7.15(\mathrm{~m}, 6 \mathrm{H}), 6.93(\mathrm{~s}, 1 \mathrm{H})$, 6.86-6.80 (m, 2H), $5.11(\mathrm{~m}, 1 \mathrm{H}), 5.00(\mathrm{dd}, J 12.3$ and $7.5 \mathrm{~Hz}, 1 \mathrm{H}$ ), 4.89 (dd, $J 12.3$ and $8.4 \mathrm{~Hz}, 1 \mathrm{H}$ ), 3.75 (s, $3 \mathrm{H}$ ); ${ }^{13} \mathrm{C}$ NMR $\left(75 \mathrm{MHz}, \mathrm{CDCl}_{3}\right) \delta 154.0,139.1,131.5,128.8$, 127.7, 127.5, 126.5, 122.3, 113.9, 112.6, 112.1, 100.7, 79.4, 55.8, 41.4; IR $v_{\max } / \mathrm{cm}^{-1}(\mathrm{KBr}) 3447(\mathrm{NH}), 1548,1482$, 1453, 1440, 1379, 1209, 1177.

5-Methoxy-3-(1-(4-methoxyphenyl)-2-nitroethyl)-1Hindole (5i): 86\%, pale yellow solid, mp 141-142 ${ }^{\circ} \mathrm{C} ;{ }^{1} \mathrm{H}$ NMR $\left(300 \mathrm{MHz}, \mathrm{CDCl}_{3}\right) \delta 8.00(\mathrm{br} \mathrm{s}, 1 \mathrm{H}), 7.28-7.21(\mathrm{~m}, 3 \mathrm{H}), 6.99$ $(\mathrm{d}, J 2.1 \mathrm{~Hz}, 1 \mathrm{H}), 6.88-6.82(\mathrm{~m}, 4 \mathrm{H}), 5.09(\mathrm{~m}, 1 \mathrm{H}), 5.02(\mathrm{dd}$, $J 11.7$ and $7.2 \mathrm{~Hz}, 1 \mathrm{H}$ ), 4.88 (dd, $J 11.7$ and $7.8 \mathrm{~Hz}, 1 \mathrm{H}$ ), $3.77(\mathrm{~s}, 6 \mathrm{H}) ;{ }^{13} \mathrm{C}$ NMR $\left(75 \mathrm{MHz}, \mathrm{CDCl}_{3}\right) \delta 158.9,154.2$, 131.6, 131.1, 128.8, 126.6, 122.1, 114.4, 114.3, 112.7, 112.1, $100.9,79.7,55.8,55.2,40.8$; IR $v_{\max } / \mathrm{cm}^{-1}(\mathrm{KBr}) 3434(\mathrm{NH})$, 2959, 1545, 1513, 1480, 1456, 1247, 1208.

3-(1-(4-Fluorophenyl)-2-nitroethyl)-5-methoxy-1Hindole (5j): 96\%, brown solid, mp 102-103 ${ }^{\circ} \mathrm{C}$; ${ }^{1} \mathrm{H}$ NMR $\left(300 \mathrm{MHz}, \mathrm{CDCl}_{3}\right) \delta 8.11$ (br s, $\left.1 \mathrm{H}\right) ; 7.28-7.20(\mathrm{~m}, 2 \mathrm{H})$, 7.17 (d, $J 8.7 \mathrm{~Hz}, 1 \mathrm{H}), 7.00-6.88$ (m, 3H), 6.83 (dd, $J 8.7$ and $2.4 \mathrm{~Hz}, 1 \mathrm{H}), 6.78(\mathrm{~d}, J 2.1 \mathrm{~Hz}, 1 \mathrm{H}), 5.08(\mathrm{~m}, 1 \mathrm{H}), 4.97$ (dd, $J 12.3$ and $7.5 \mathrm{~Hz}, 1 \mathrm{H}$ ), 4.83 (dd, $J 12.3$ and $8.4 \mathrm{~Hz}$, $1 \mathrm{H}), 3.74(\mathrm{~s}, 3 \mathrm{H}) ;{ }^{13} \mathrm{C} \mathrm{NMR}\left(75 \mathrm{MHz}, \mathrm{CDCl}_{3}\right) \delta 161.9(\mathrm{~d}$, $J 2.4 \mathrm{~Hz}), 154.0,134.8(\mathrm{~d}, J 3 \mathrm{~Hz}), 131.5,129.3(\mathrm{~d}, J 8 \mathrm{~Hz})$, 126.3, 122.1, $115.7(\mathrm{~d}, J 21 \mathrm{~Hz}), 113.6,112.6,112.2,100.7$, 79.4, 55.7, 40.7; IR $v_{\max } / \mathrm{cm}^{-1}(\mathrm{KBr}) 3434(\mathrm{NH}), 1551,1509$, 1482, 1454, 1439, 1377, 1226, 1207, 808.

4-(1-(5-Methoxy-1H-indol-3-yl)-2-nitroethyl)- $N, N$ dimethylaniline $(\mathbf{5} \boldsymbol{k})$ : $87 \%$, pink solid, $\mathrm{mp} 134-135^{\circ} \mathrm{C}$; ${ }^{1} \mathrm{H}$ NMR (300 MHz, $\left.\mathrm{CDCl}_{3}\right) \delta 7.99(\mathrm{br} \mathrm{s}, 1 \mathrm{H}), 7.23$ (d, $J 8.1 \mathrm{~Hz}, 1 \mathrm{H}), 7.21-7.14(\mathrm{~m}, 2 \mathrm{H}), 6.96(\mathrm{~d}, J 2.4 \mathrm{~Hz}$, $1 \mathrm{H}), 6.87(\mathrm{~d}, J 2.4 \mathrm{~Hz}, 1 \mathrm{H}), 6.83(\mathrm{dd}, J 8.7$ and $2.4 \mathrm{~Hz}$, $1 \mathrm{H}), 6.68(\mathrm{~d}, J 9 \mathrm{~Hz}, 2 \mathrm{H}), 5.07-4.95(\mathrm{~m}, 2 \mathrm{H}), 4.92-4.80$ $(\mathrm{m}, 1 \mathrm{H}), 3.77(\mathrm{~s}, 3 \mathrm{H}), 2.90(\mathrm{~s}, 6 \mathrm{H}) ;{ }^{13} \mathrm{C}$ NMR $(75 \mathrm{MHz}$, $\left.\mathrm{CDCl}_{3}\right) \delta 154.0,149.7,131.6,128.4,126.9,126.6,122.2$, 114.7, 112.9, 112.5, 112.0, 101.0, 79.8, 55.8, 40.7, 40.6; IR $v_{\max } / \mathrm{cm}^{-1}(\mathrm{KBr}) 3408(\mathrm{NH}), 1616,1541,1524,1484$, 1376, 1210, 1201. HRMS (ESI ${ }^{+}$) Found: 340.16545. Calc. for $\mathrm{C}_{19} \mathrm{H}_{21} \mathrm{O}_{3} \mathrm{~N}_{3}: 340.16557$.

3-(1-(3,4-Dimethoxyphenyl)-2-nitroethyl)-5-methoxylH-indole (5l): $98 \%$, brownish solid, mp 131-132 ${ }^{\circ} \mathrm{C}$; ${ }^{1} \mathrm{H}$ NMR $\left(300 \mathrm{MHz}, \mathrm{CDCl}_{3}\right) \delta 8.31(\mathrm{br} \mathrm{s}, 1 \mathrm{H}), 7.15(\mathrm{~d}$, $J 8.7 \mathrm{~Hz}, 1 \mathrm{H}), 6.94-6.74(\mathrm{~m}, 6 \mathrm{H}), 5.06(\mathrm{~m}, 1 \mathrm{H}), 4.98(\mathrm{dd}$, $J 11.7$ and $7.2 \mathrm{~Hz}, 1 \mathrm{H}), 4.86(\mathrm{dd}, J 11.7$ and $8.4 \mathrm{~Hz}, 1 \mathrm{H}$ ), 3.79 (s, 3H), 3.76 (s, 3H), 3.75 (s, 3H); ${ }^{13} \mathrm{C}$ NMR $(75 \mathrm{MHz}$,
$\left.\mathrm{CDCl}_{3}\right) \delta 153.9,149.0,148.1,131.6,131.5,126.4,122.2$, 119.6, 113.8, 112.3, 112.1, 111.2, 111.1, 100.7, 79.5, 55.7, 55.7, 55.6, 41.1; IR $v_{\max } / \mathrm{cm}^{-1}(\mathrm{KBr}) 3436(\mathrm{NH}), 2955$, 2931, 2830, 1544, 1510, 1259, 1020. HRMS (ESI $\left.{ }^{+}, m / \mathrm{Na}^{+}\right)$ Found: 379.12635. Calc. for $\mathrm{C}_{19} \mathrm{H}_{20} \mathrm{O}_{5} \mathrm{~N}_{2} / \mathrm{Na}^{+}$: 379.12644.

5-Methoxy-3-(1-(naphthalen-1-yl)-2-nitroethyl)-1Hindole (5m): $98 \%$, brown solid, mp 76-77 ${ }^{\circ} \mathrm{C} .{ }^{1} \mathrm{H}$ NMR (300 MHz, $\left.\mathrm{CDCl}_{3}\right) \delta 8.25(\mathrm{~d}, J 8.1 \mathrm{~Hz}, 1 \mathrm{H}), 7.97$ (br s, 1H), 7.92-7.74 (m, 2H), 7.58-7.17 (m, 5H), 6.95-6.81 (m, 3H), $6.00(\mathrm{t}, J 7.8 \mathrm{~Hz}, 1 \mathrm{H}), 5.08$ (d, J 7.8 Hz, 2H), $3.71(\mathrm{~s}, 3 \mathrm{H})$; ${ }^{13} \mathrm{C} \mathrm{NMR}\left(75 \mathrm{MHz}, \mathrm{CDCl}_{3}\right) \delta 154.2,134.6,134.2,131.7$, $131.2,129.1,128.3,126.8,126.6,125.9,125.3,124.5$, 123.3, 122.7, 114.0, 112.7, 112.1, 100.7, 78.5, 55.8, 37.0; IR $v_{\text {max }} / \mathrm{cm}^{-1}(\mathrm{KBr}) 3420(\mathrm{NH}), 1549,1484,1457,1437$, 1376, 1213, 800 .

5-Methoxy-3-(2-nitro-1-(thiophen-2-yl)ethyl)-1Hindole $(\mathbf{5 n})$ : $93 \%$, greenish brown solid, $\mathrm{mp} 83-85^{\circ} \mathrm{C}$; ${ }^{1} \mathrm{H} \mathrm{NMR}\left(300 \mathrm{MHz}, \mathrm{CDCl}_{3}\right) \delta 8.15$ (br s, $\left.1 \mathrm{H}\right), 7.26-7.20$ $(\mathrm{m}, 2 \mathrm{H}), 7.06-6.89(\mathrm{~m}, 5 \mathrm{H}), 5.45(\mathrm{~m}, 1 \mathrm{H}), 5.04(\mathrm{dd}$, $J 12.6$ and $7.5 \mathrm{~Hz}, 1 \mathrm{H}), 4.99$ (dd, $J 12.6$ and $8.1 \mathrm{~Hz}$, $1 \mathrm{H}), 3.85(\mathrm{~s}, 3 \mathrm{H}) ;{ }^{13} \mathrm{C} \mathrm{NMR}\left(75 \mathrm{MHz}, \mathrm{CDCl}_{3}\right) \delta 154.1$, 142.9, 131.4, 126.9, 126.1, 125.1, 124.8, 113.5, 112.6, 112.2, 100.6, 79.9, 55.8, 36.8; IR v $\mathrm{vax}_{\max } / \mathrm{cm}^{-1}(\mathrm{KBr}) 3336$ (NH), 2929, 2829, 1551, 1489, 1436, 1376, 1214, 716; HRMS (ESI ${ }^{+}, m / \mathrm{Na}^{+}$) Found: 325.06167. Calc. for $\mathrm{C}_{15} \mathrm{H}_{14} \mathrm{O}_{3} \mathrm{~N}_{2} \mathrm{~S} / \mathrm{Na}^{+}: 325.06174$.

2-Methyl-3-(2-nitro-1-phenylethyl)-1H-indole (5o):67 94\%, pale pink solid, mp 102-103 ${ }^{\circ} \mathrm{C} ;{ }^{1} \mathrm{H}$ NMR $(300 \mathrm{MHz}$, $\left.\mathrm{CDCl}_{3}\right) \delta 7.80(\mathrm{br} \mathrm{s}, 1 \mathrm{H}), 7.35(\mathrm{~d}, J 7.8 \mathrm{~Hz}, 1 \mathrm{H}), 7.32-7.16$ $(\mathrm{m}, 6 \mathrm{H}), 7.08(\mathrm{~m}, 1 \mathrm{H}), 7.00(\mathrm{~m}, 1 \mathrm{H}), 5.23-5.04(\mathrm{~m}, 3 \mathrm{H})$, $2.28(\mathrm{~s}, 3 \mathrm{H}) ;{ }^{13} \mathrm{C}$ NMR $\left(75 \mathrm{MHz}, \mathrm{CDCl}_{3}\right) \delta 139.5,135.3$, $132.8,128.7$; 127.2, 127.0, 126.8, 121.2, 119.6, 118.5, 110.7, 108.7, 78.6, 40.4, 11.8; IR $v_{\max } / \mathrm{cm}^{-1}(\mathrm{KBr}) 3403$ (NH), 1550, 1459, 1428, 1377, 743, 700.

2-Methyl-3-(1-(naphthalen-1-yl)-2-nitroethyl)- $1 \mathrm{H}$ indole (5p): $94 \%$, pale yellow solid, $\mathrm{mp} 150-151{ }^{\circ} \mathrm{C}$; ${ }^{1} \mathrm{H} \mathrm{NMR}\left(300 \mathrm{MHz}, \mathrm{CDCl}_{3}\right) \delta 8.11(\mathrm{~m}, 1 \mathrm{H}), 7.89-7.82$ (m, 2H), $7.79(\mathrm{~d}, J 8.1 \mathrm{~Hz}, 1 \mathrm{H}), 7.60(\mathrm{~d}, J 7.2 \mathrm{~Hz}, 1 \mathrm{H})$, 7.55 (d, J 7.2 Hz, 1H), 7.52-7.39 (m, 3H), 7.28-7.21 (m, $1 \mathrm{H}), 7.15-7.03(\mathrm{~m}, 2 \mathrm{H}), 5.92(\mathrm{~m}, 1 \mathrm{H}), 5.25(\mathrm{dd}, J 12.9$ and $6.9 \mathrm{~Hz}, 1 \mathrm{H}), 5.19(\mathrm{dd}, J 12.9$ and $8.7 \mathrm{~Hz}, 1 \mathrm{H}), 2.27(\mathrm{~s}, 3 \mathrm{H})$; ${ }^{13} \mathrm{C} \mathrm{NMR}\left(75 \mathrm{MHz}, \mathrm{CDCl}_{3}\right) \delta 135.3,134.5,134.1,132.9$, 131.3, 129.1, 128.3, 127.3, 126.6, 125.8, 125.1, 124.3, 123.0, 121.4, 119.8, 118.4, 110.7, 108.3, 78.0, 37.4, 12.3; IR $v_{\text {max }} / \mathrm{cm}^{-1}(\mathrm{KBr}) 3415,3348,2921,2870,1453,903$, 742; HRMS (ESI ${ }^{+}, m / \mathrm{Na}^{+}$) Found: 353.12611. Calc.for $\mathrm{C}_{21} \mathrm{H}_{18} \mathrm{O}_{2} \mathrm{~N}_{2} / \mathrm{Na}^{+}: 353.12605$. 
2-Methyl-3-(2-nitro-1-(thiophen-2-yl)ethyl)-1H-indole $(5 q)$ : 98\%, greenish brown solid, mp $89-91{ }^{\circ} \mathrm{C}{ }^{1} \mathrm{H}$ NMR $\left(300 \mathrm{MHz}, \mathrm{CDCl}_{3}\right) \delta 7.75($ br s, $1 \mathrm{H}) ; 7.32(\mathrm{~d}, J 7.8 \mathrm{~Hz}$, $1 \mathrm{H}), 7.16-6.95$ (m, 4H), 6.87-6.80 (m, 2H), 5.29 (dd, J 9.0 and $6.6 \mathrm{~Hz}, 1 \mathrm{H}), 5.10(\mathrm{dd}, J 12.3$ and $6.6 \mathrm{~Hz}, 1 \mathrm{H}), 4.99$ $(\mathrm{dd}, J 12.3$ and $9.0 \mathrm{~Hz}, 1 \mathrm{H}) ;{ }^{13} \mathrm{C} \mathrm{NMR}\left(75 \mathrm{MHz}, \mathrm{CDCl}_{3}\right)$ $\delta$ 143.2, 135.2, 133.0, 126.7, 126.1, 124.6, 124.2, 121.1, $119.5,118.4,110.7,108.1,78.7,36.4,11.4 ;$ IR $v_{\max } / \mathrm{cm}^{-1}$ (neat, film) $3404(\mathrm{NH}), 1551,1460,1427,1378,745$, 702; HRMS (ESI ${ }^{+}, m / \mathrm{Na}^{+}$) Found: 309.06671. Calc. for $\mathrm{C}_{15} \mathrm{H}_{14} \mathrm{O}_{2} \mathrm{~N}_{2} \mathrm{~S} / \mathrm{Na}^{+}: 309.06682$.

\section{Preparation of the triptamine derivative 8}

To a stirred solution of compound 5a $(266.3 \mathrm{mg}$, $1 \mathrm{mmol})$ in $\mathrm{CH}_{3} \mathrm{OH}(5 \mathrm{~mL})$ were sequentially added $\mathrm{Pd} / \mathrm{C}$ $10 \%(52 \mathrm{mg})$ and $\mathrm{HCOONH}_{4}(315.3 \mathrm{mg}, 5 \mathrm{mmol})$. The reaction mixture was stirred at room temperature overnight. The reaction mixture was filtered on a celite pad, washed several times with $\mathrm{CH}_{3} \mathrm{OH}$ and the solvent removed on a rotatory evaporator. To the white residue was added saturated $\mathrm{Na}_{2} \mathrm{CO}_{3}(\mathrm{aq})$ until $\mathrm{pH} 8$ was reached and then the aqueous mixture was extracted twice with EtOAc. The combined organic phases were dried over $\mathrm{MgSO}_{4}$, filtered and the solvent removed under vacuum, affording compound $\mathbf{8}$. The crude product was used in the next step without further purification.

2-(1H-Indol-3-yl)-2-phenylethanamine (8): ${ }^{58} 90 \%$, pale brown solid, mp $129-130{ }^{\circ} \mathrm{C} .{ }^{1} \mathrm{H}$ NMR $\left(300 \mathrm{MHz}, \mathrm{CDCl}_{3}\right.$ ) $\delta 8.33(\mathrm{br} \mathrm{s}, 1 \mathrm{H}), 7.45(\mathrm{~d}, J 7.8 \mathrm{~Hz}, 1 \mathrm{H}), 7.35-7.10(\mathrm{~m}, 7 \mathrm{H})$, $7.02(\mathrm{t}, J 7.5 \mathrm{~Hz}, 2 \mathrm{H}), 4.25(\mathrm{~m}, 1 \mathrm{H}), 3.42(\mathrm{dd}, J 12.6$ and $7.2 \mathrm{~Hz}, 1 \mathrm{H}), 3.27$ (dd, $J 12.6$ and $7.8 \mathrm{~Hz}, 1 \mathrm{H}), 1.71$ (br s, $2 \mathrm{H}) ;{ }^{13} \mathrm{C}$ NMR $\left(75 \mathrm{MHz}, \mathrm{CDCl}_{3}\right) \delta 142.9,136.5,128.5$, 128.1, 127.0, 126.4, 122.1, 121.3, 119.4, 119.3, 117.4, 111.1, 47.1, 46.7; IR $v_{\max } / \mathrm{cm}^{-1}$ (KBr) 3415, 3348, 2921, 2870, 1453, 903, 742 .

\section{Preparation of $\beta$-carboline derivative 9}

To a suspension of $\mathrm{MgSO}_{4}(240 \mathrm{mg})$ and compound $8(70.9 \mathrm{mg}, 0.3 \mathrm{mmol})$ in $\mathrm{CH}_{2} \mathrm{Cl}_{2}(2 \mathrm{~mL})$ was added benzaldehyde ( $37 \mu \mathrm{L}, 0.36 \mathrm{mmol}, 1.2$ equiv.) at room temperature and the mixture was stirred at room temperature for $1 \mathrm{~h}$. The suspension was cooled to $0{ }^{\circ} \mathrm{C}$ and TFA $(45 \mu \mathrm{L}$ $0.6 \mathrm{mmol}, 2.0$ equiv.) was slowly added. The reaction was stirred at room temperature for $72 \mathrm{~h}$. The reaction was quenched by addition of sat. $\mathrm{NaHCO}_{3}$ at $0{ }^{\circ} \mathrm{C}$ until pH 8 was reached. After filtration over celite, the filtrate was extracted with $\mathrm{CH}_{2} \mathrm{Cl}_{2}$. The combined organic layers were washed with brine, dried over anhydrous $\mathrm{MgSO}_{4}$, filtered and the solvent evaporated under vacuum. The ${ }^{1} \mathrm{H}$ NMR analysis of the crude product showed a mixture of two diastereoisomers in 87:13 ratio. The 1,4-trans relative configuration for the major isomer was assumed based on literature data. The crude product was purified by column chromatography using a mixture of hexanes/ethyl acetate 75:25 (v/v) as eluent. After purification only the major isomer was observed in the ${ }^{1} \mathrm{H}$ NMR spectra.

trans-1,4-Diphenyl-2,3,4,9-tetrahydro- $1 \mathrm{H}$ pyrido[3,4-b] indole (9-trans): ${ }^{59} 70 \%$, pale brown solid, mp 184-185 ${ }^{\circ} \mathrm{C}$; ${ }^{1} \mathrm{H}$ NMR (300 MHz, $\mathrm{CDCl}_{3}$ ) $\delta 7.70$ (br s, $1 \mathrm{H}), 7.40-7.21(\mathrm{~m}, 10 \mathrm{H}), 7.18(\mathrm{~d}, J 8.1 \mathrm{~Hz}, 1 \mathrm{H}), 7.06(\mathrm{~m}$, $1 \mathrm{H}), 6.90-6.78(\mathrm{~m}, 2 \mathrm{H}), 5.25(\mathrm{~s}, 1 \mathrm{H}), 4.37(\mathrm{~m}, 1 \mathrm{H}), 3.53$ (dd, $J 12.6$ and $5.1 \mathrm{~Hz}, 1 \mathrm{H}$ ), 3.02 (dd, $J 12.6$ and $8.7 \mathrm{~Hz}$, 1H), 1.91 (br s, $1 \mathrm{H}) ;{ }^{13} \mathrm{C} \mathrm{NMR}\left(75 \mathrm{MHz}, \mathrm{CDCl}_{3}\right) \delta 143.0$, 141.4, 135.9, 135.8, 128.9, 128.6, 128.4, 128.3, 126.7, 126.5, 121.5, 119.8, 119.3, 112.5, 110.7, 58.2, 52.9, 41.7; IR $v_{\max } / \mathrm{cm}^{-1}(\mathrm{KBr}) 3405,3319,3052,3019,2952,2811$, 1451, 1273, 750, 697.

\section{Supplementary Information}

The spectroscopic ${ }^{1} \mathrm{H}$ NMR, ${ }^{13} \mathrm{C}$ NMR, IR data as well as the HRMS of selected compounds are provided as supplementary information and available free of charge at http:// jbcs.sbq.org.br, as PDF file.

\section{Acknowledgments}

The authors thank the Conselho Nacional de Desenvolvimento Científico e Tecnológico (CNPq) for the financial support (D. R., Grant Universal No. 484615/2007-6). C. S. S. acknowledges the Coordenação de Aperfeiçoamento de Pessoal de Nível Superior (CAPES) for the graduate fellowship. We would like to thank Prof. Dr. Marcos N. Eberlin and MSc Clécio F. Klitzke from the Thomson Mass Spectrometry Laboratory of Instituto de Química-UNICAMP, for the HRMS analysis, and also Prof. Dr. Luiz Carlos Dias for his generosity reading and suggesting improvements to this work.

\section{References}

1. Gilchrist, T. L.; Heterocyclic Chemistry, Academic Press: London, 1997.

2. Sundberg, R. J.; Indoles, Academic Press: San Diego, 1996.

3. Sundberg, R. J.; The Chemistry of Indoles, Academic Press: New York, 1970.

4. Bandini, M., Umani-Ronchi, A.; Catalytic Asymmetric FriedelCrafts Alkylations, Wiley-VCH: Weinheim, 2009. 
5. Poulsen, T. B.; Jørgensen, K. A.; Chem. Rev. 2008, 108, 2903.

6. Sheng, Y. F.; Zhang, A. J.; Zheng, X. J.; You, S. L.; Chin. J. Org. Chem. 2008, 28, 605.

7. Harrington, P. E.; Kerr, M. A.; Synlett 1996, 1047.

8. Sakagami, M.; Muratake, H.; Natsume, M.; Chem. Pharm. Bull. 1994, 42, 1393.

9. Fukuyama, T.; Chen, X.; J. Am. Chem. Soc. 1994, 116, 3125.

10. Vaillancourt, V.; Albizati, K. F.; J. Am. Chem. Soc. 1993, 115, 3499 .

11. Murakatake, H.; Kumagami, H.; Natsume, M.; Tetrahedron 1990, 46, 6351.

12. Houlihan, W. J.; Indoles, John Wiley \& Sons Inc: New York, 1972, vol. I.

13. Yadav, J. S.; Abraham, S.; Reddy, B. V. S.; Sabitha, G.; Synthesis 2001, 2165.

14. Bandini, M.; Melchiorre, P.; Melloni, A.; Umani-Ronchi, A.; Synthesis 2002, 1110.

15. Zhan, Z. P.; Yang, R. F.; Lang, K.; Tetrahedron Lett. 2005, 46, 3859.

16. Huang, Z. H.; Zou, H. P.; Jiang, W. Q.; Tetrahedron Lett. 2006, 47, 7965 .

17. Maiti, G.; Kundu, P.; Synth. Commun. 2007, 37, 2309.

18. Kawatsura, M.; Aburatani, S.; Uenishi, J.; Tetrahedron 2007, 63,4172 .

19. Li, J. T.; Liu, Z. P.; Liu, C. T.; Indian J. Chem., Sect B: Org. Chem. Incl. Med. Chem. 2008, 47, 283.

20. Kumar, V.; Kaur, S.; Kumar, S.; Tetrahedron Lett. 2006, 47, 7001.

21. Kawatsura, M.; Aburatani, S.; Uenishi, J.; Synlett 2005, 2492.

22. Alam, M. M.; Varala, R.; Adapa, S. R.; Tetrahedron Lett. 2003, 44,5115 .

23. Shi, M.; Cui, S.-C.; Li, Q. J.; Tetrahedron 2004, 60, 6679.

24. Arcadi, A.; Bianchi, G.; Chiarini, M.; D’Anniballe, G.; Marinelli, F.; Synlett 2004, 944.

25. Firouzabadi, H.; Iranpoor, N.; Jafarpour, M.; Ghaderi, A.; J. Mol. Catal. A: Chem. 2006, 252, 150.

26. Tabatabaeian, K.; Mamaghani, M.; Mahmoodi, N. O.; Khorshidi, A.; J. Mol. Catal. A: Chem. 2007, 270, 112.

27. Itoh, T.; Uehara, H.; Ogiso, K.; Nomura, S.; Hayase, S.; Kawatsura, M.; Chem. Lett. 2007, 36, 50.

28. Russowsky, D.; Petersen, R. Z.; Godoi, M. N.; Pilli, R. A.; Tetrahedron Lett. 2000, 41, 9939.

29. Andrade, C. K. Z.; Rocha, R. O.; Russowsky, D.; Godoy, M. N.; J. Braz. Chem. Soc. 2005, 16, 535.

30. Silveira, C. C.; Vieira, A. S.; Braga, A. L.; Russowsky, D.; Tetrahedron 2005, 61, 9312.

31. Godoi, M. N.; Costenaro, H. S.; Kramer, E.; Machado, P. S.; D’Oca, M. G. M.; Russowsky, D.; Quim Nova 2005, 28, 1010.

32. Russowsky, D.; Canto, R. F. S.; Sanches, S. A. A.; D’Oca, M. G. M.; De Fatima, A.; Pilli, R. A.; Kohn, L. K.; Antonio, M. A.; De Carvalho, J. E.; Bioorg. Chem. 2006, 34, 173.
33. Russowsky, D.; Benvenutti, E. V.; Roxo, G. S.; Grasel, F.; Lett. Org. Chem. 2007, 4, 39.

34. Ceschi, M. A.; Peppe, C.; Felix, L. A.; Tetrahedron Lett. 2000, 41, 9695 .

35. Ceschi, M. A.; Schenato, R. A.; Pisoni, D.; Borges, D.; J. Braz. Chem. Soc. 2004, 15, 652.

36. Costa, J. S.; Pisoni, D. S.; Silva, C. B.; Petzhold, C. L.; Russowsky, D.; Ceschi, M. A.; J. Braz. Chem. Soc. 2009, 20, 1448.

37. Russowsky, D.; Lopes, F. A.; Da Silva, V. S. S.; Canto, K. F. S.; D’Oca, M. G. M.; Godoi, M. N.; J. Braz. Chem. Soc. 2004, $15,165$.

38. Ford, K. L.; Roskamp, E. J.; Tetrahedron Lett. 1992, 33, 1135.

39. Arumugam, P.; Perumal, P. T.; Chem. Lett. 2006, 35, 632.

40. Cayley, A. N.; Cox, R. J.; Menard-Moyon, C.; Schmidt, J. P.; Taylor, R. J. K.; Tetrahedron Lett. 2007, 48, 6556.

41. Wabnitz, T. C.; Yu, J. Q.; Spencer, J. B.; Chem. Eur. J. 2004, 10, 484.

42. Kobayashi, S.; Nagayama, S.; Busujima, T.; J. Am. Chem. Soc. 1998, 120, 8287; Kobayashi, S.; Manabe, K.; Acc. Chem. Res. 2002, 35, 209.; Kobayashi, S.; Sugiura, M.; Kitagawa, H.; Lam, W. W. L.; Chem. Rev. 2002, 102, 2227.

43. Fringuelli, F.; Pizzo, F.; Vaccaro, L.; J. Org. Chem. 2001, 66, 4719.

44. Aplander, K.; Ding, R.; Krasavin, M.; Lindström, U.M.; Wennerberg, J.; Eur. J. Org. Chem. 2009, 810.

45. Cardoso, A. L.; Neves, S. C. G.; Silva, M. J.; Energies 2008, 1, 79; Silva, M. J.; Cardoso, A. L. Natalino, R.; Int. J. Chem. Reactor Eng. 2010, 8, A12.

46. Bandini, M.; Cozzi, P. G.; Giacomini, M.; Melchiorre, P.; Selva, S.; Umani-Ronchi, A.; J. Org. Chem. 2002, 67, 3700.

47. Pavia, D. L.; Lampman, G. M.; Kriz, G. S.; Engel, R. G.; Introduction to Organic Laboratories Techniques: A Small Scale Aproach, 15t. ed., Brooks/Cole: Pacific Grove, 1998.

48. Bartoli, G.; Bosco, M.; Giuli, S.; Giuliani, A.; Lucarelli, L.; Marcantoni, A.; Sambri, L.; Torregiani, E.; J. Org. Chem. 2005, 70, 1941.

49. Bandini, M.; Garelli, A.; Rovinetti, M.; Tommasi, S.; UmaniRonchi, A.; Chirality 2005, 17, 522.

50. Ballini, R.; Petrini, M.; ARKIVOC 2009, 9, 195.

51. Ballini, R.; Palmieri, A.; Curr. Org. Chem. 2006, 10, 2145.

52. Ono, N.; The Nitro Group in Organic Synthesis, Wiley-VCH: Weinheim, 2001.

53. Bellamy, F. D.; Ou, K. ; Tetrahedron Lett. 1984, 25, 839.

54. Tanaka, K.; Toda, F.; Chem. Rev. 2000, 100, 1025.

55. Gawande, M. B.; Polshettiwar, V.; Varma, S.R.; Jayaram, R.V.; Tetrahedron Lett. 2007, 48, 8170.

56. Tanaka, K.; Solvent-Free Organic Synthesis, Wiley-VCH Verlag GmbH \& Co. KgaA Eds.: Weinheim, 2003.

57. Ford, P. W.; Narbut, M. R.; Belli, J.; Davidson, B. S.; J. Org. Chem. 1994, 59, 5955. 
58. Herrera, R. P.; Sgarzani, V.; Bernardi, L.; Ricci, A.; Angew. Chem., Int. Ed. 2005, 44, 6576.

59. Itoh, J.; Fuchibe, K.; Akiyama, T.; Angew. Chem., Int. Ed. 2008, 47, 4016.

60. Bartoli, G.; Bosco, M.; Carlone, A.; Pesciaioli, F.; Sambri, L.; Melchiorre, P.; Org. Lett. 2007, 9, 1403.

61. Gu, Y. L.; Ogawa, C.; Kobayashi, S.; Org. Lett. 2007, 9, 175.

62. Adachi, S.; Tanaka, F.; Watanabe, K.; Harada, T.; Org. Lett. 2009, 11, 5206.

63. Chen, W.; Du, W.; Yue, L.; Li, R.; Wu, Y.; Ding, L. S.; Chen, Y. C.; Org. Biomol. Chem. 2007, 5, 816.
64. Zhou, W.; Xu, L. W.; Yang, L.; Zhao, L. Q.; Xia, C. G.; J. Mol. Catal. A: Chem. 2006, 249, 129.

65. Maiti, G.; Kundu, P.; Synth. Commun. 2007, 37, 2309.

66. Damodiran, M.; Kumar, R. S.; Sivakumar, P. M.; Doble, M.; Perumal, P. T.; J. Chem. Sci. (Bangalore, India) 2009, 121, 65.

67. Lin, S. Z.; You, T. P.; Tetrahedron 2009, 65, 1010.

68. An, L. T.; Zou, J. P.; Zhang, L. L.; Zhang, Y.; Tetrahedron Lett. 2007, 48, 4297.

69. Liu, H.; Lu, S. F.; Xu, J; Du, D. M.; Chem. Asian J. 2008, 3, 1111.

Submitted: July 31, 2010

Published online: November 23, 2010 


\section{Supplementary Information}

\section{Metal Halide Hydrates as Lewis Acid Catalysts for the Conjugated Friedel-Crafts Reactions of Indoles and Activated Olefins}

Cristiane S. Schwalm, Marco Antonio Ceschi and Dennis Russowsky*

Instituto de Química, Universidade Federal do Rio Grande do Sul, Av. Bento Gonçalves 9500,

91501-970 Porto Alegre-RS, Brazil<smiles>CCC(=O)CC(C)c1c[nH]c2ccc(OC)cc12</smiles>

Figure S1. ${ }^{1} \mathrm{H} \mathrm{NMR}\left(\mathrm{CDCl}_{3}\right)$ of compound 3f. 
<smiles>CCC(=O)CC(C)c1c[nH]c2ccc(OC)cc12</smiles>

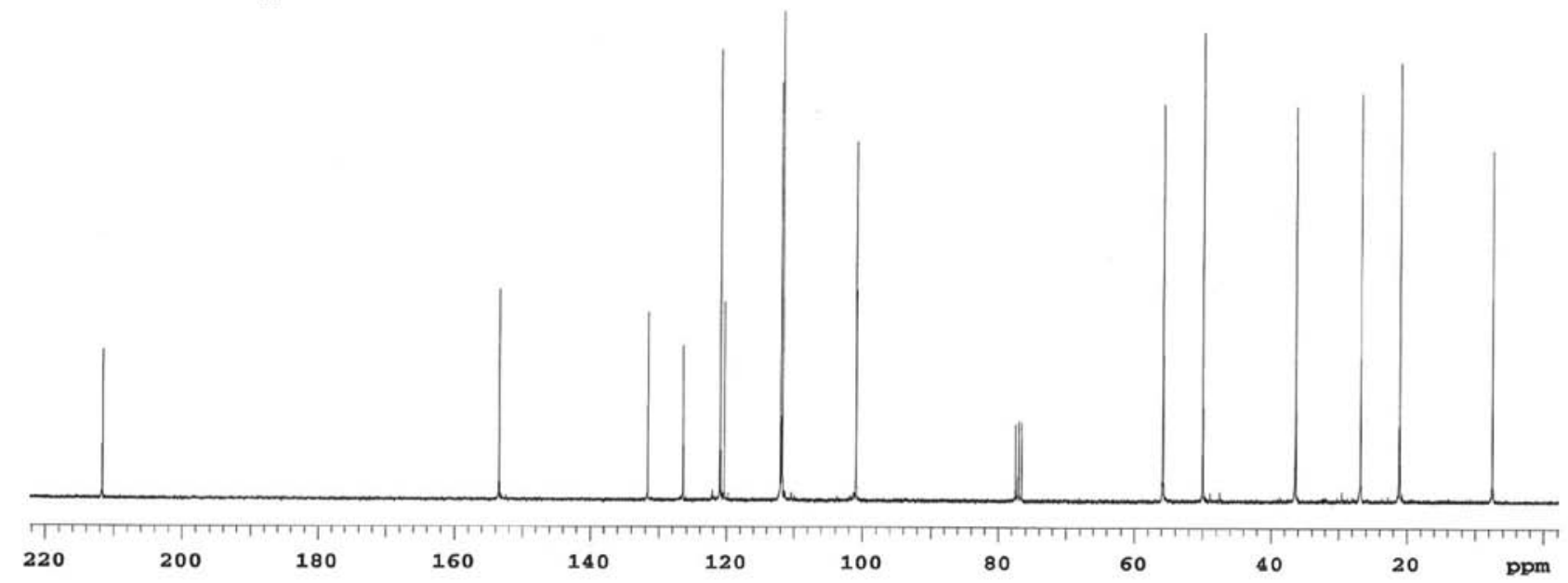

Figure S2. ${ }^{13} \mathrm{C}$ NMR $\left(\mathrm{CDCl}_{3}\right)$ of compound $\mathbf{3 f}$.

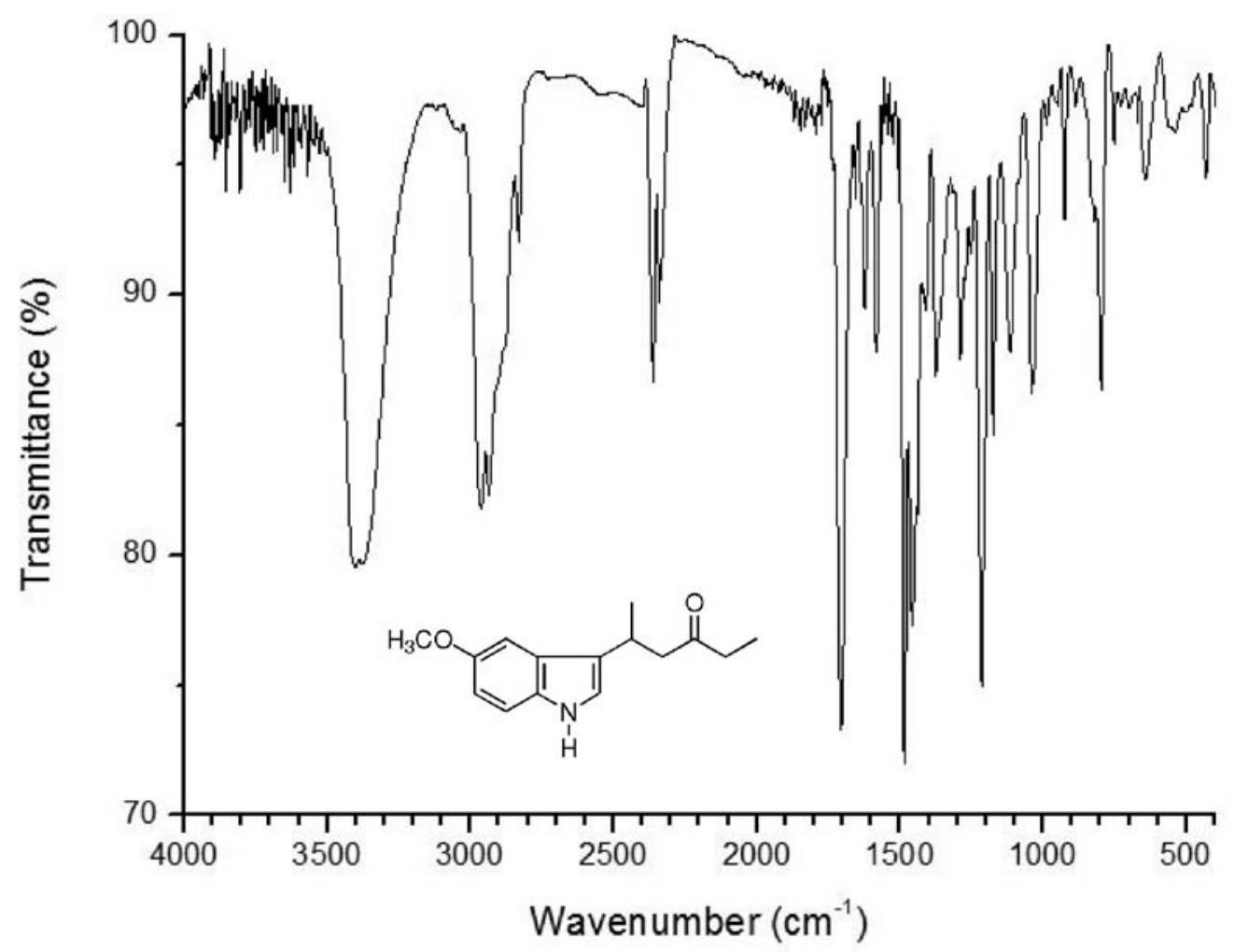

Figure S3. IR (neat) of compound 3f. 


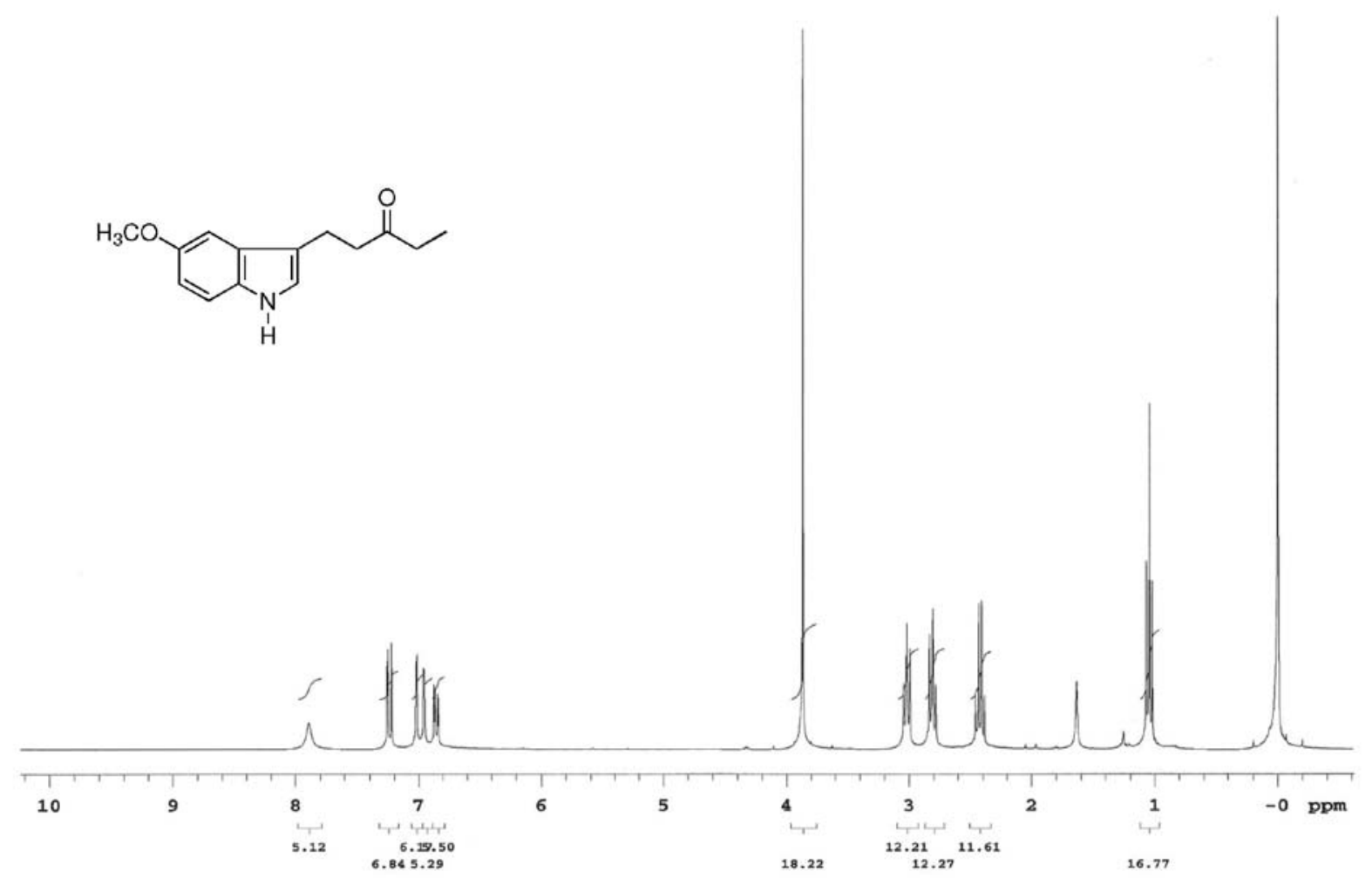

Figure S4. ${ }^{1} \mathrm{H} \mathrm{NMR}\left(\mathrm{CDCl}_{3}\right)$ of compound $\mathbf{3 g}$

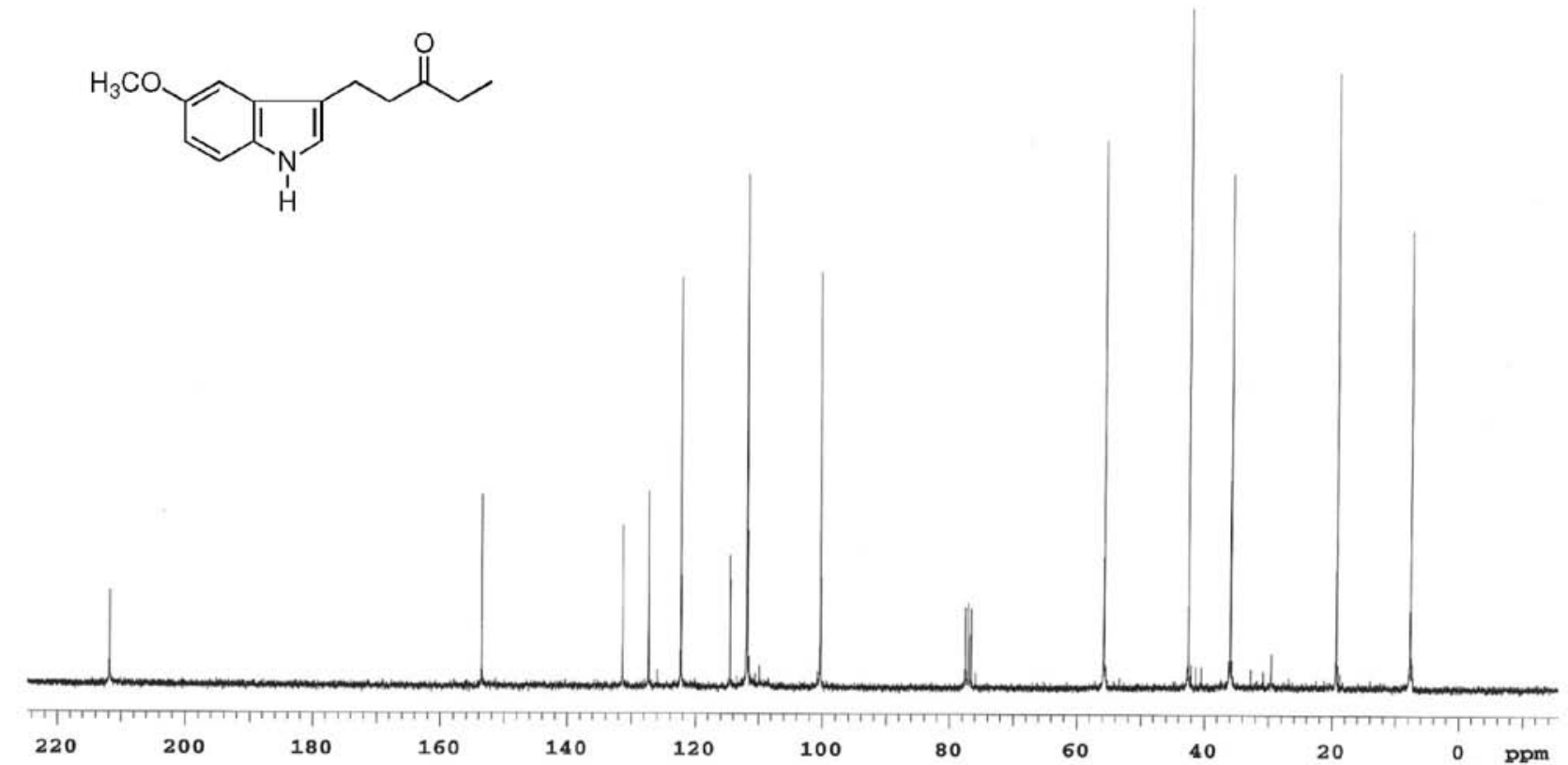

Figure S5. ${ }^{13} \mathrm{C} \mathrm{NMR}\left(\mathrm{CDCl}_{3}\right)$ of compound $\mathbf{3 g}$ 


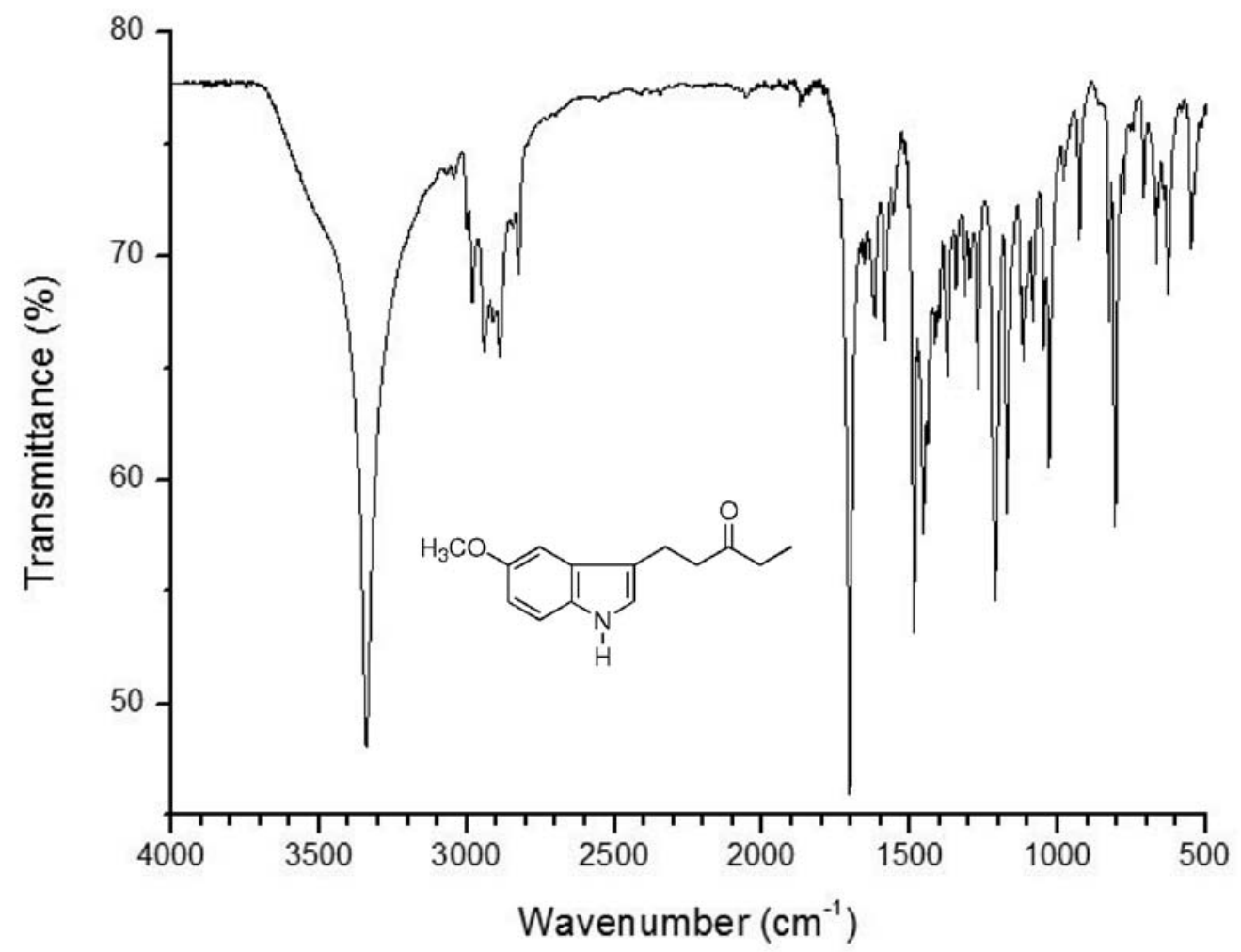

Figure S6. IR ( $\mathrm{KBr}$ ) of compound 3g.

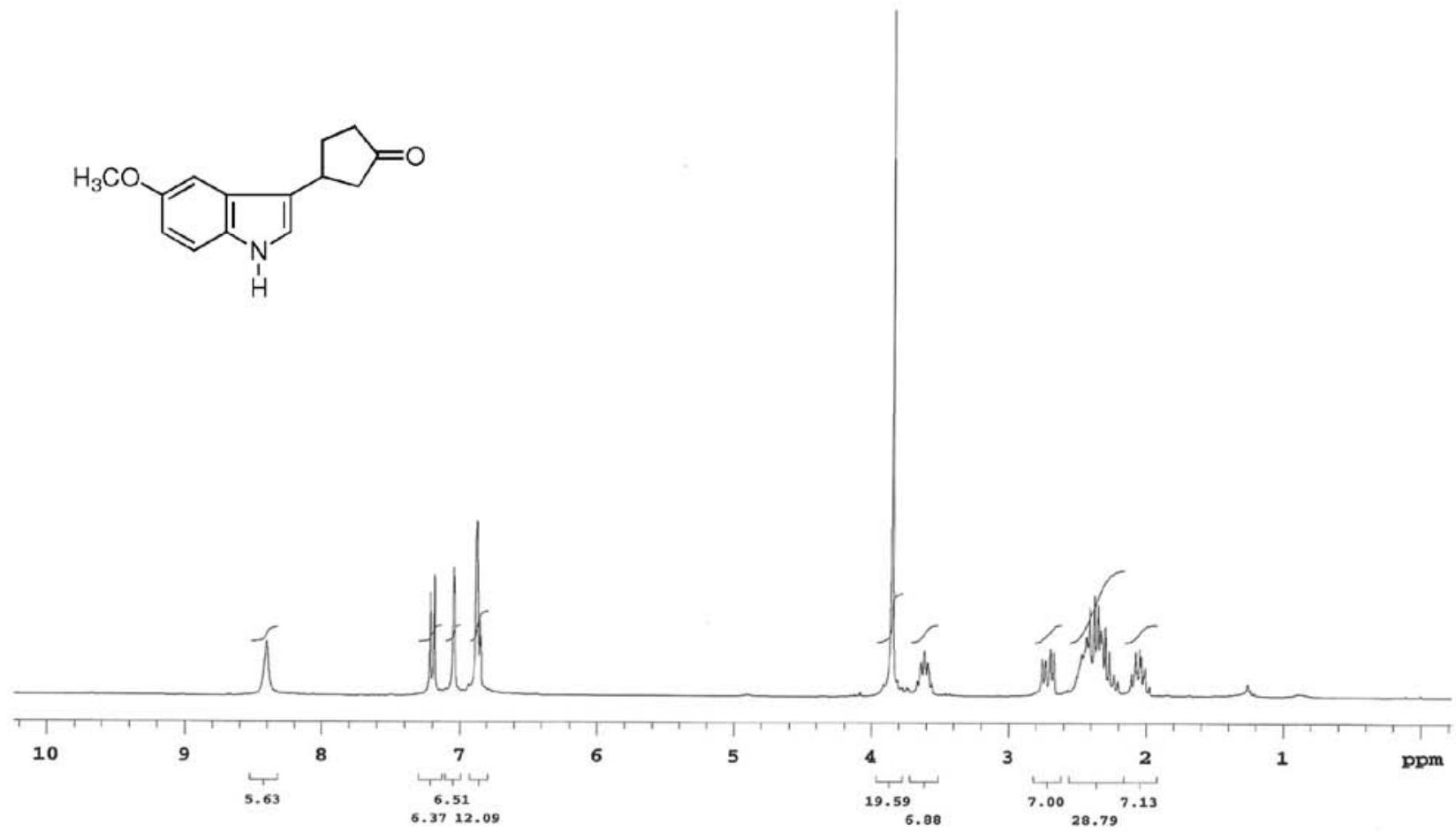

Figure S7. ${ }^{1} \mathrm{H}$ NMR $\left(\mathrm{CDCl}_{3}\right)$ of compound $\mathbf{3 i}$. 


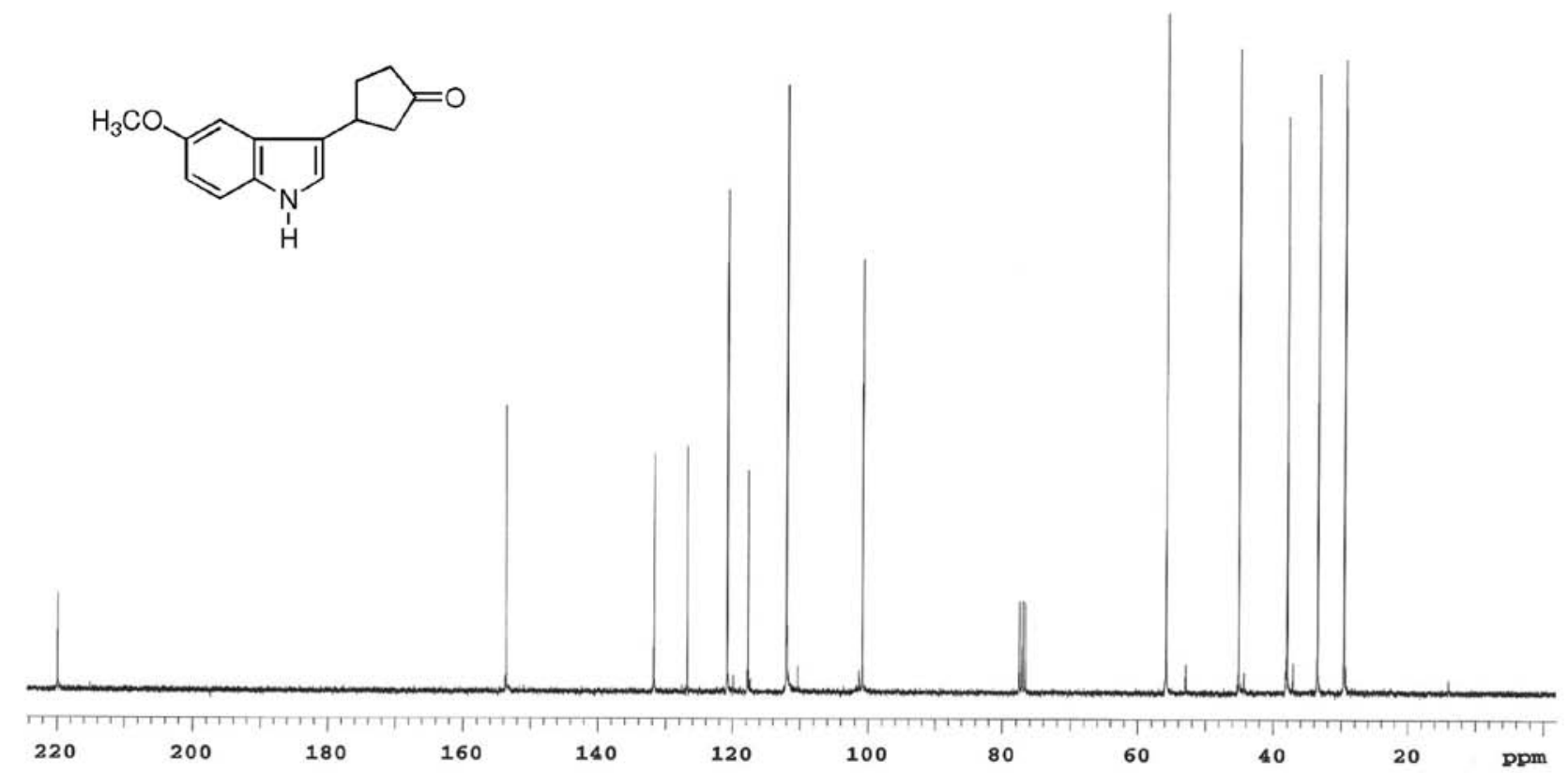

Figure S8. ${ }^{13} \mathrm{C}$ NMR $\left(\mathrm{CDCl}_{3}\right)$ of compound 3i

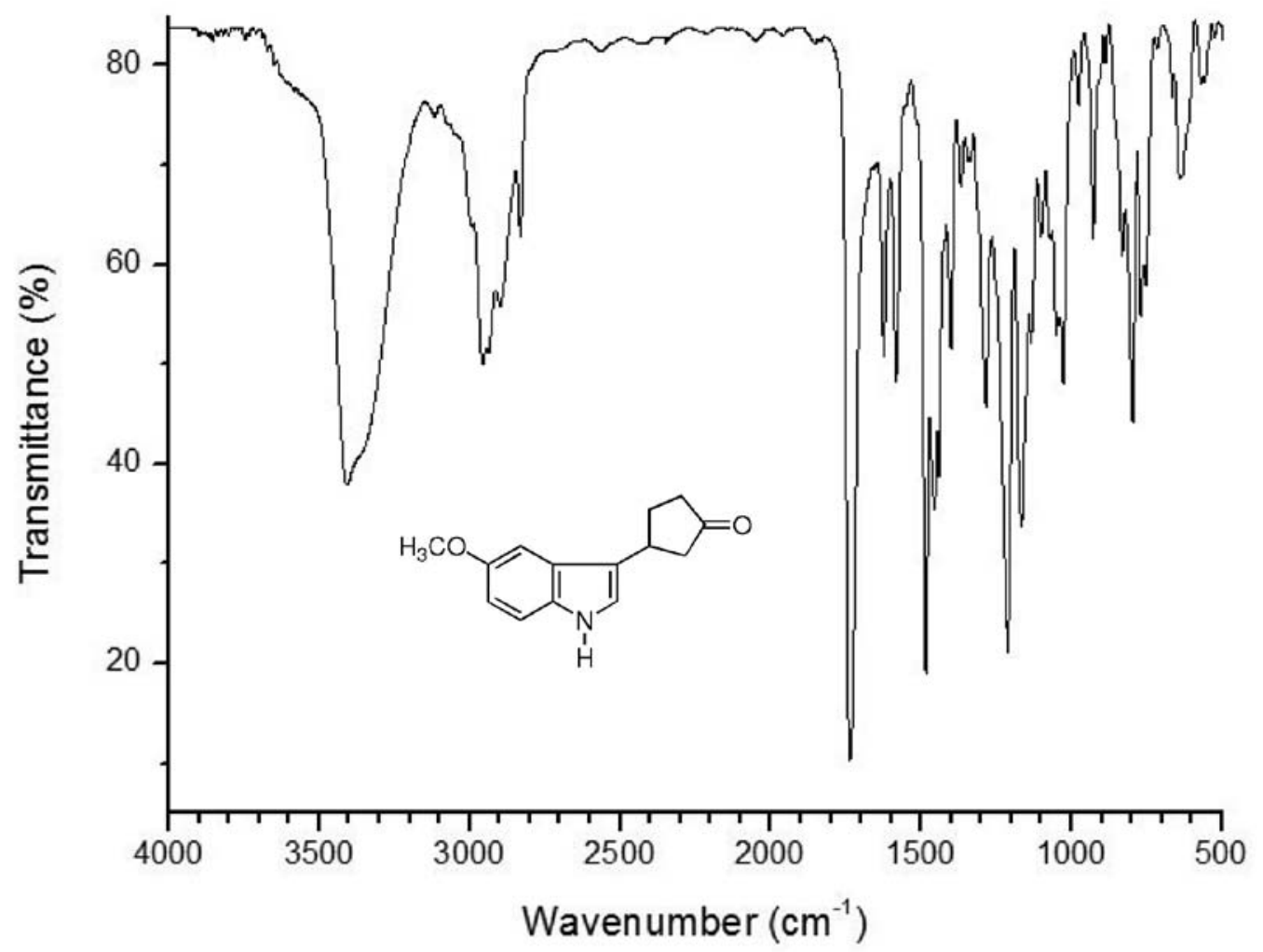

Figure S9. IR (neat) of compound 3i. 
<smiles>CCC(=O)CC(C)c1c(C)[nH]c2ccccc12</smiles>

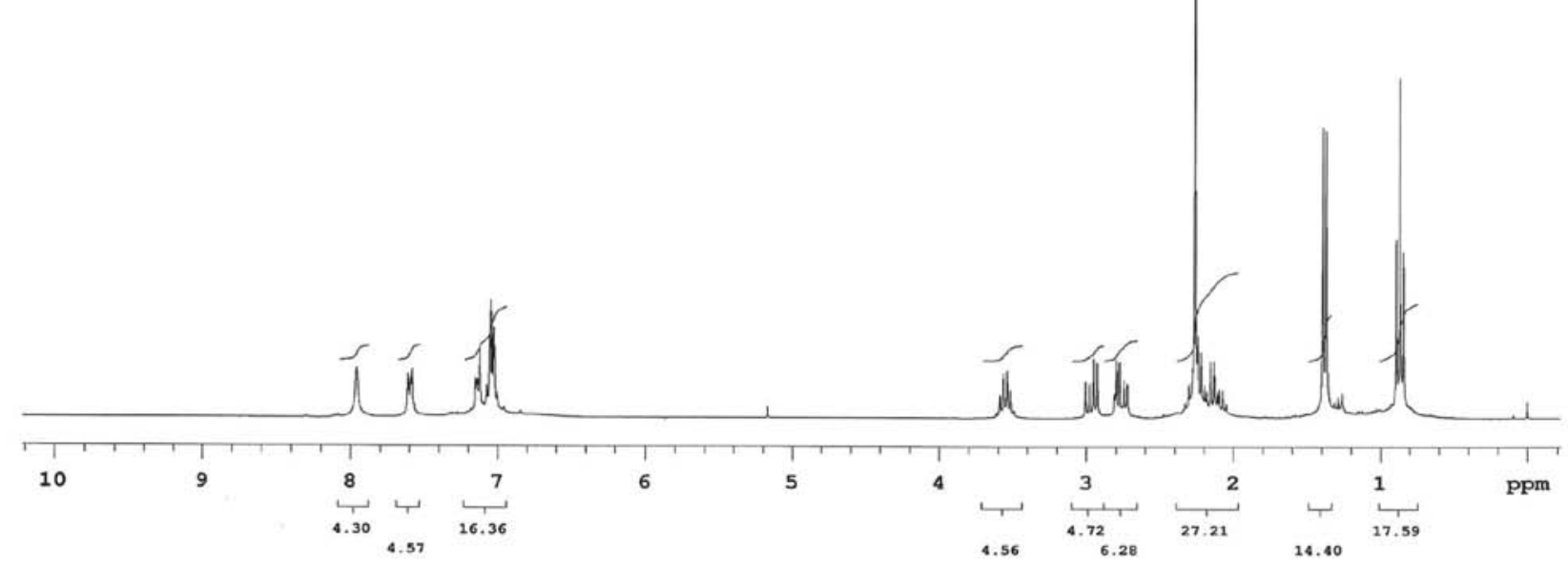

Figure S10. ${ }^{1} \mathrm{H}$ NMR $\left(\mathrm{CDCl}_{3}\right)$ of compound $3 \mathbf{k}$.<smiles>CCC(=O)CC(C)c1c(C)[nH]c2ccccc12</smiles>

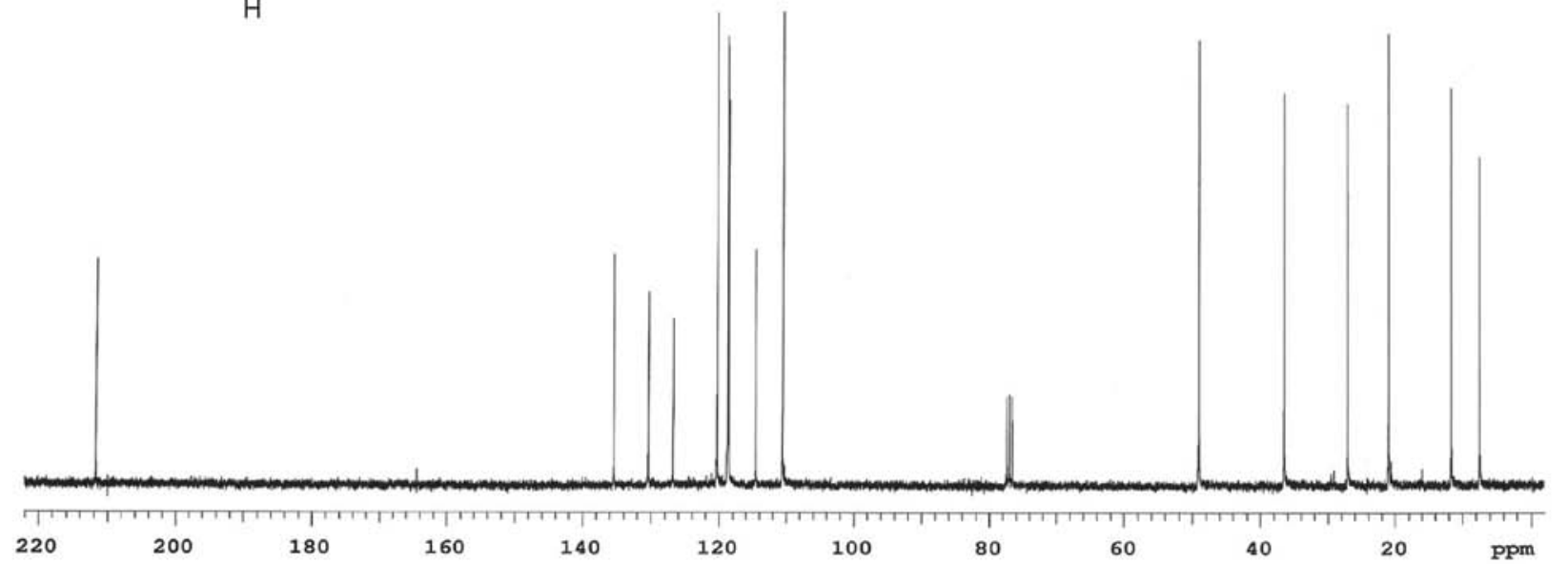

Figure S11. ${ }^{13} \mathrm{C} \mathrm{NMR}\left(\mathrm{CDCl}_{3}\right)$ of compound 3k. 


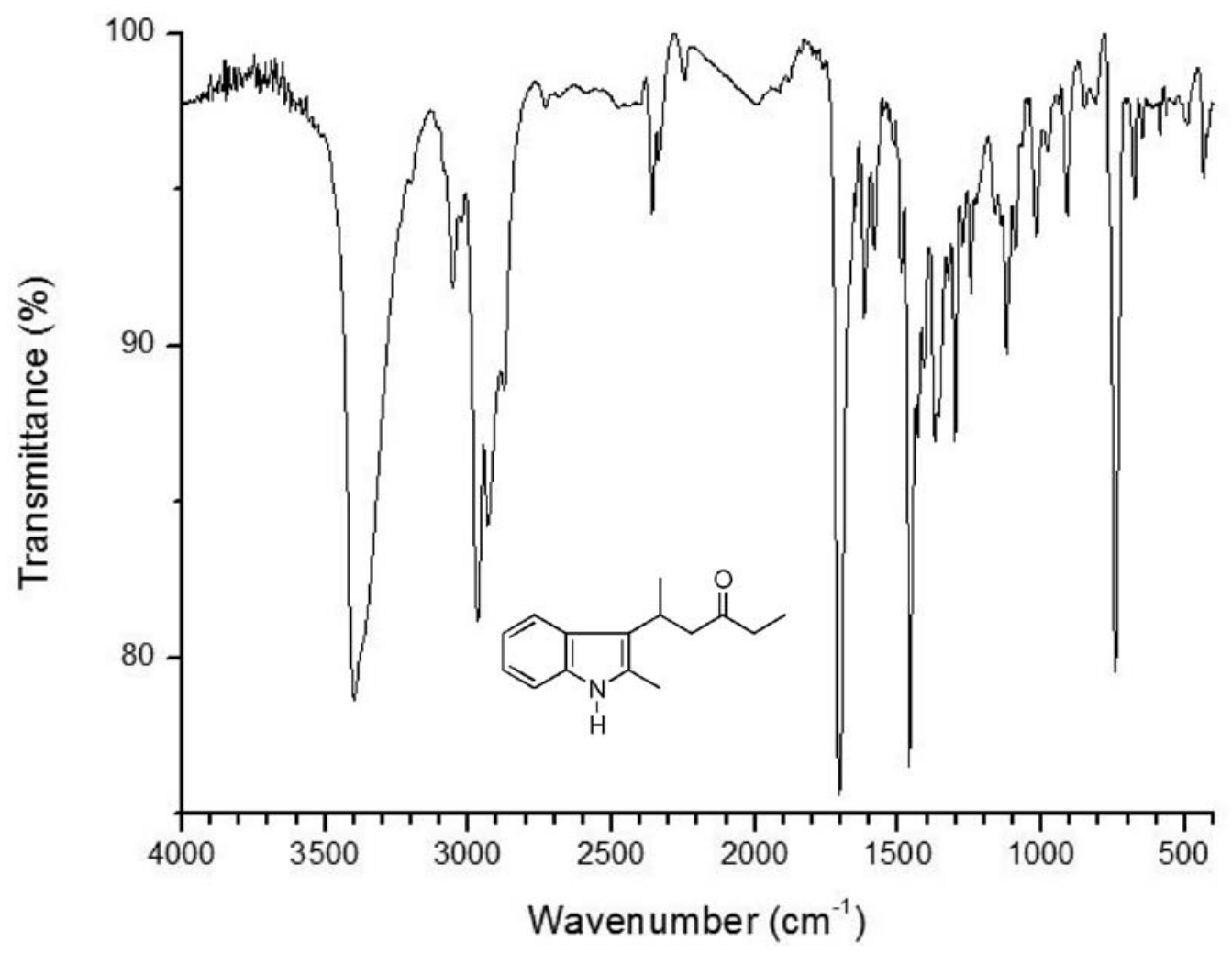

Figure S12. IR (neat) of compound 3k.<smiles>CCC(=O)CCc1c(C)[nH]c2ccccc12</smiles>

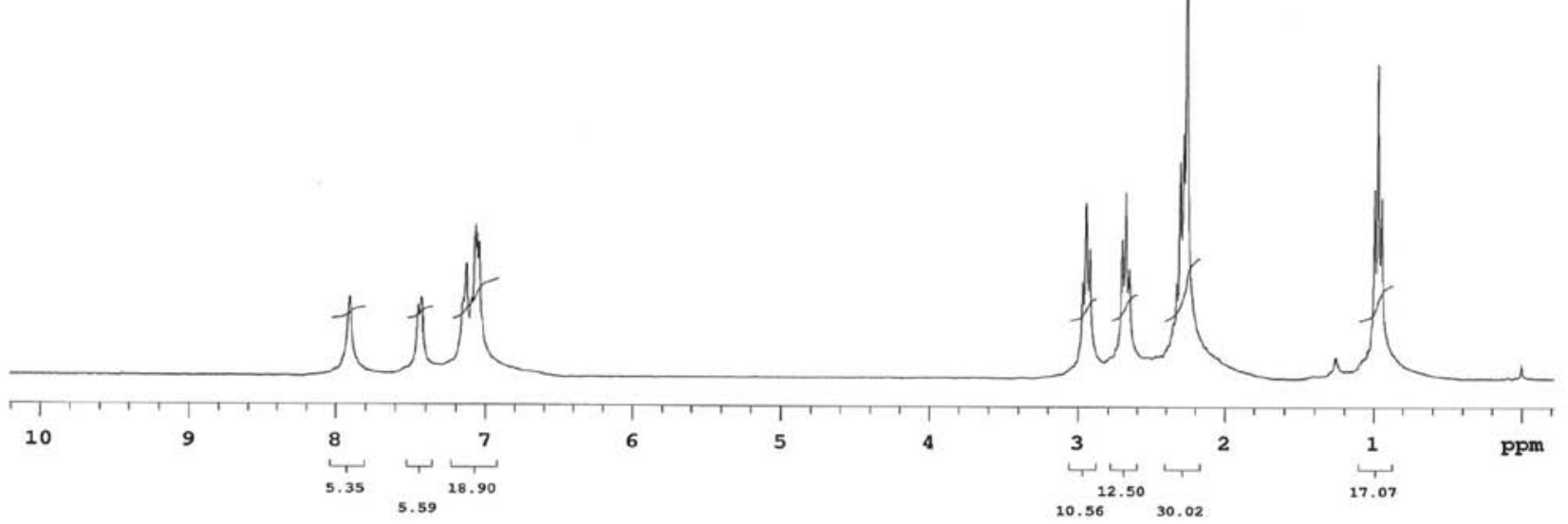

Figure S13. ${ }^{1} \mathrm{H} \mathrm{NMR}\left(\mathrm{CDCl}_{3}\right)$ of compound 31. 
<smiles>CCC(=O)CCc1c(C)[nH]c2ccccc12</smiles>

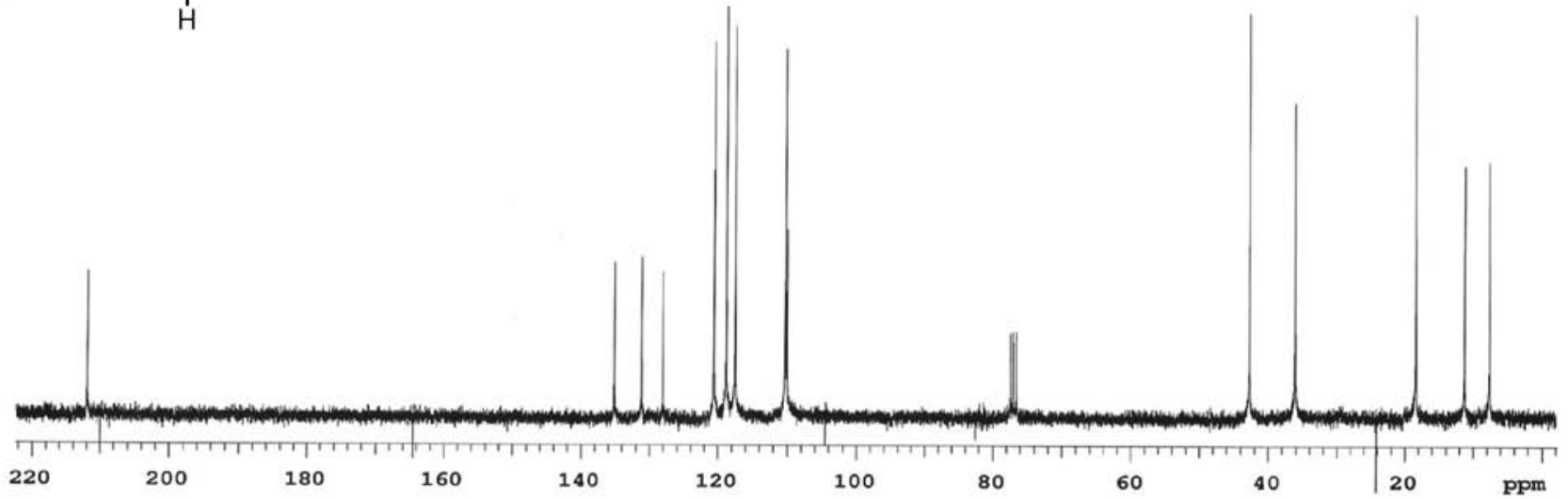

Figure S14. ${ }^{13} \mathrm{C}$ NMR $\left(\mathrm{CDCl}_{3}\right)$ of compound 31 .

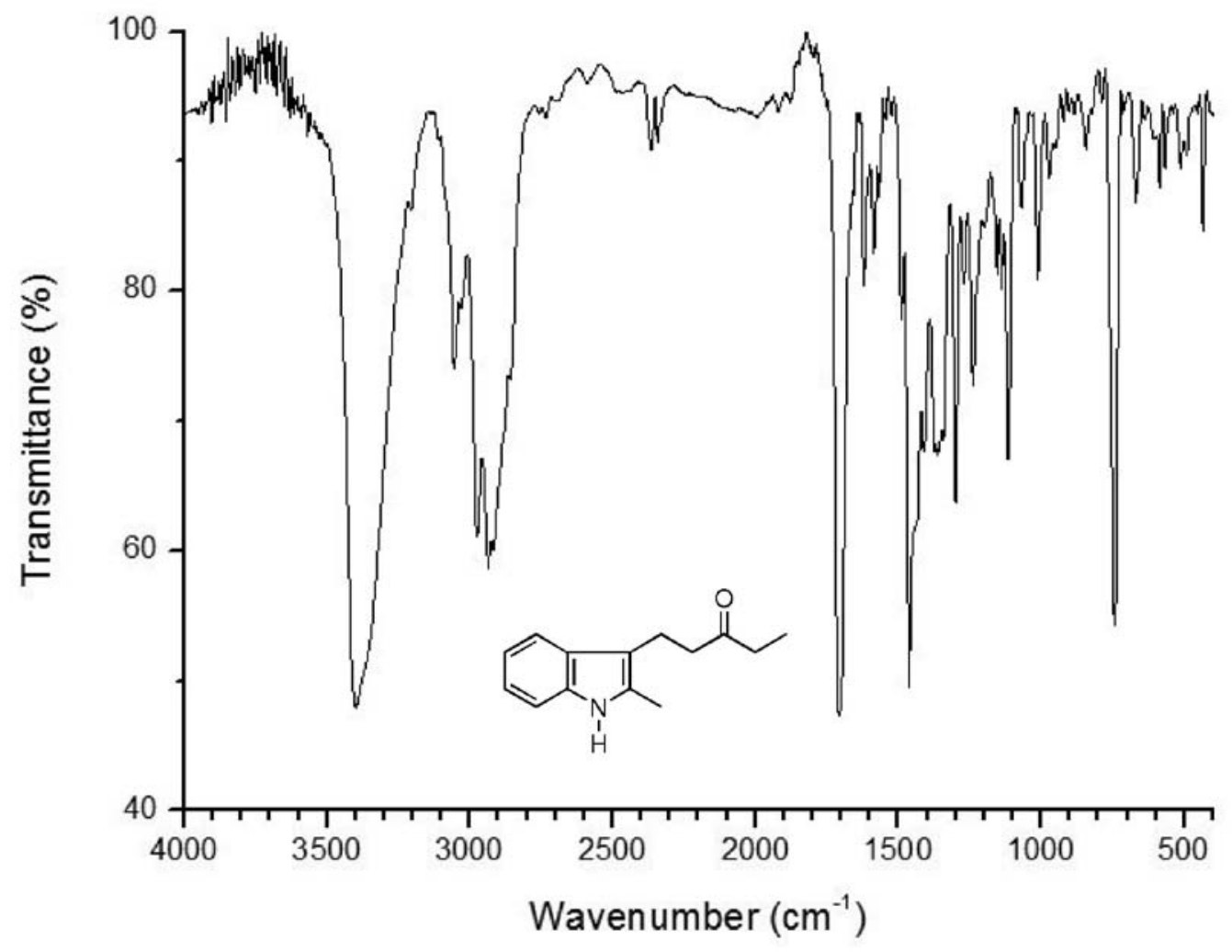

Figure S15. IR (neat) of compound 31. 
Vol. 22, No. 4, 2011

Schwalm et al.

S9

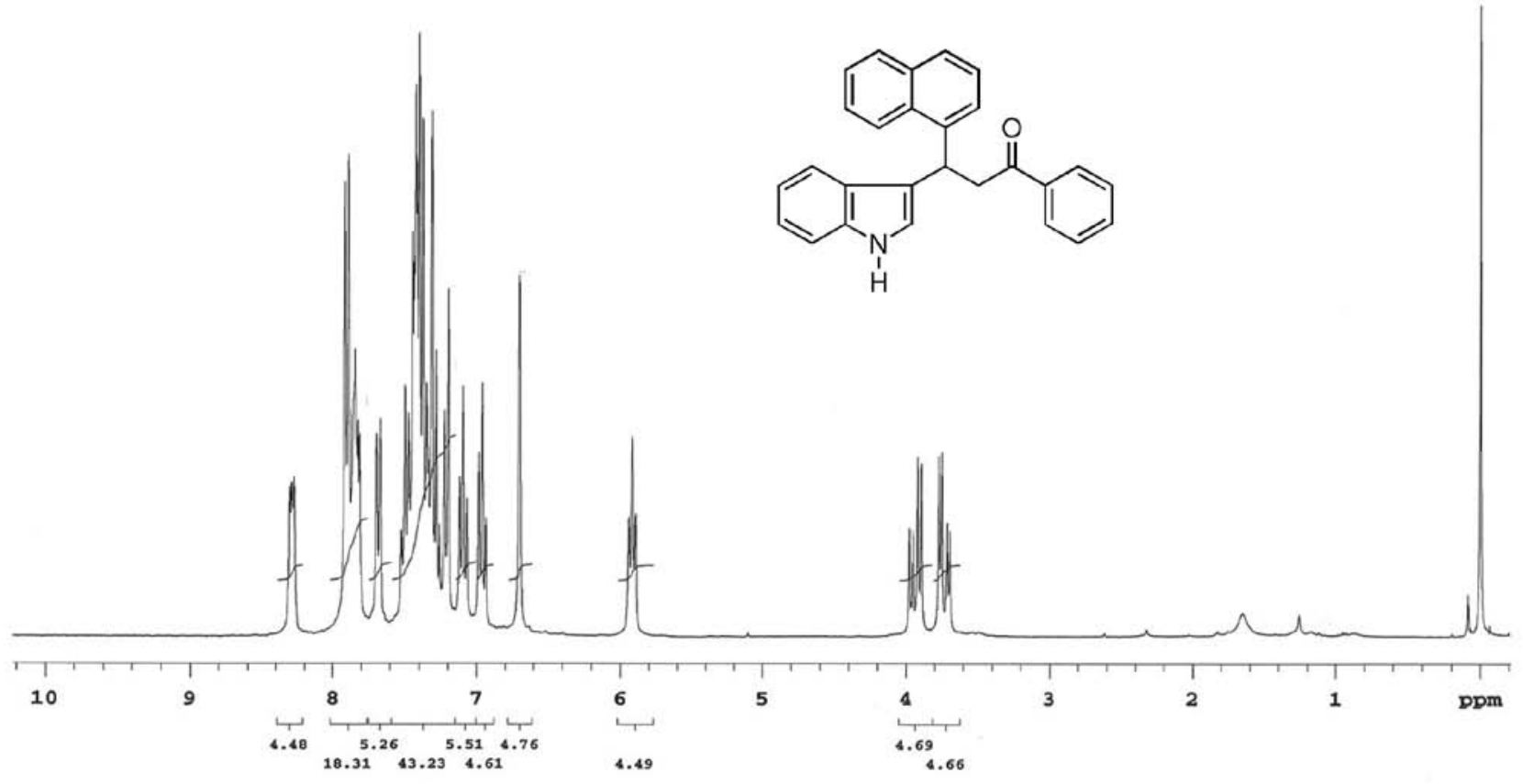

Figure S16. ${ }^{1} \mathrm{H} \mathrm{NMR}\left(\mathrm{CDCl}_{3}\right)$ of compound 3s.

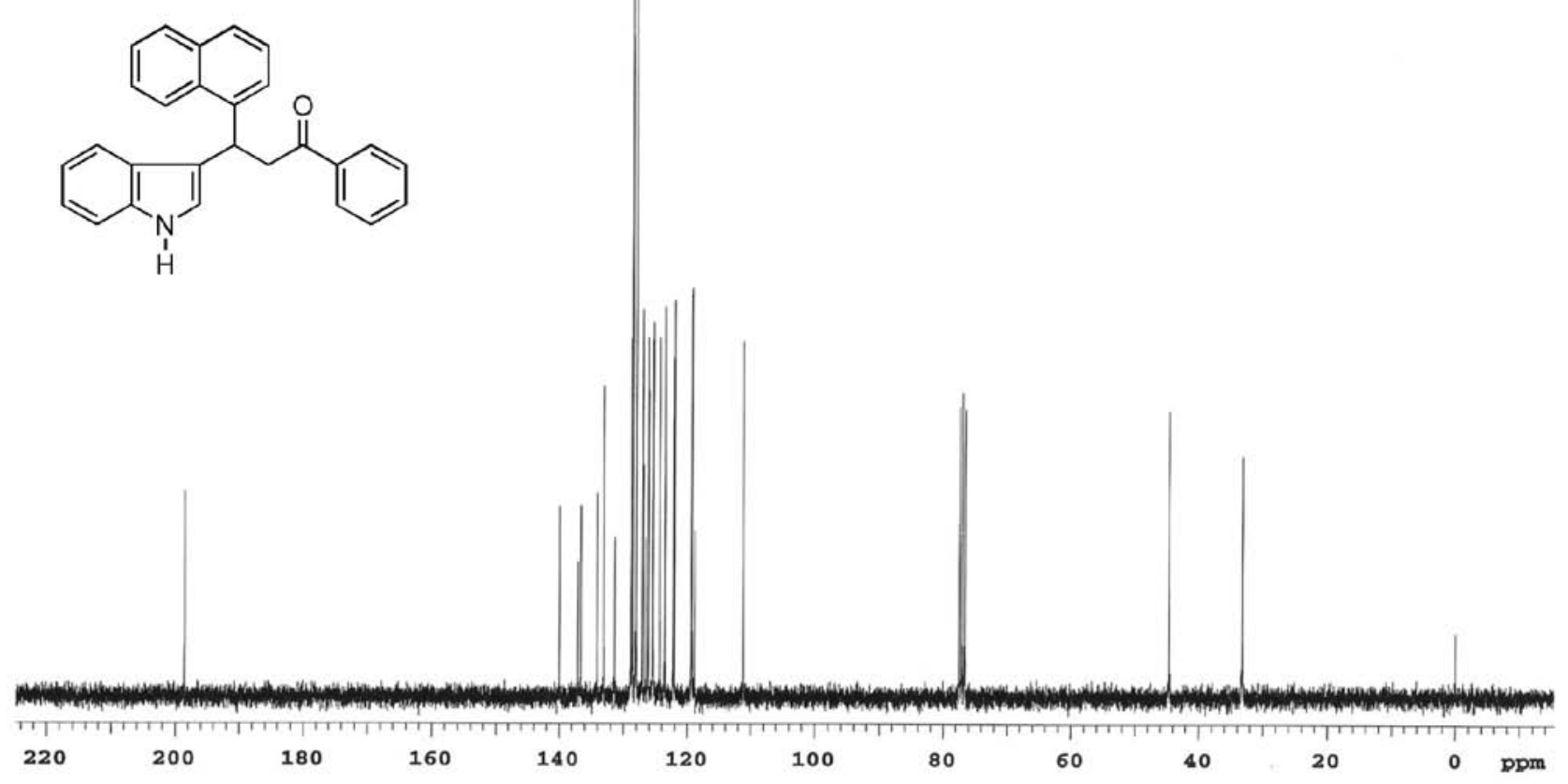

Figure S17. ${ }^{13} \mathrm{C} \mathrm{NMR}\left(\mathrm{CDCl}_{3}\right)$ of compound 3s. 
S10

Metal Halide Hydrates as Lewis Acid Catalysts for the Conjugated Friedel-Crafts Reactions

J. Braz. Chem. Soc.

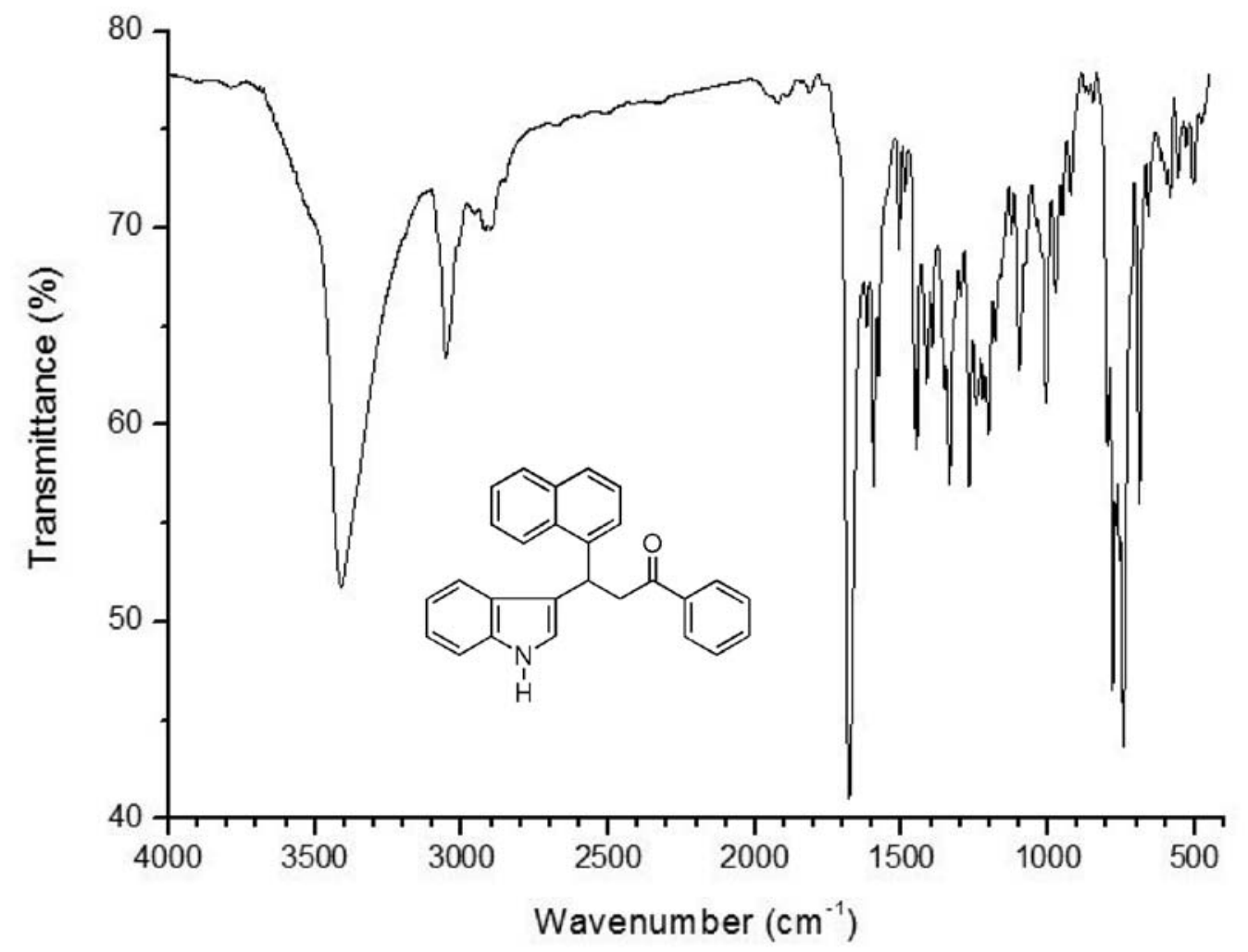

Figure S18. IR ( $\mathrm{KBr})$ of compound Bs.
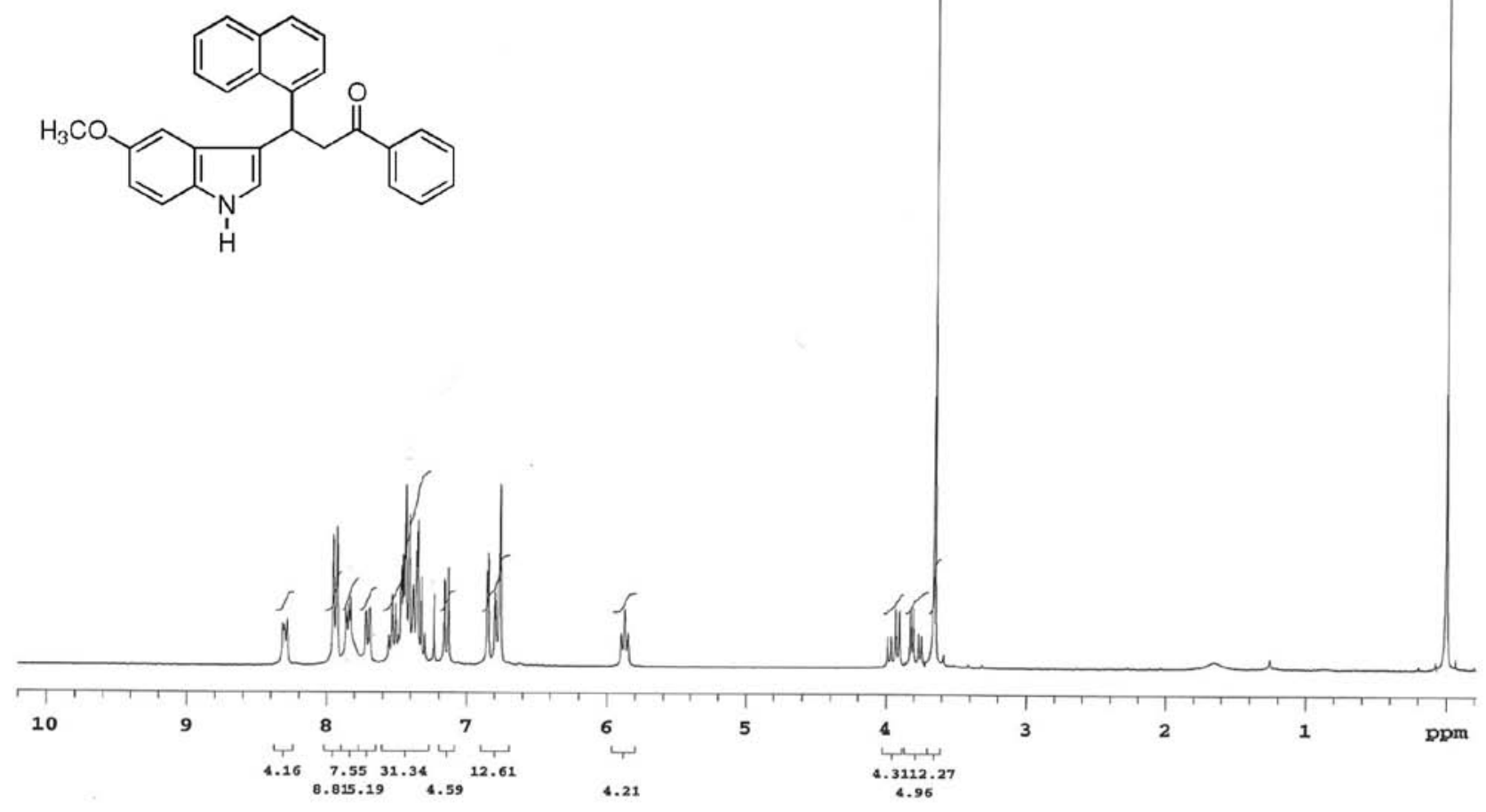

Figure S19. ${ }^{1} \mathrm{H} \mathrm{NMR}\left(\mathrm{CDCl}_{3}\right)$ of compound $\mathbf{3 w}$. 


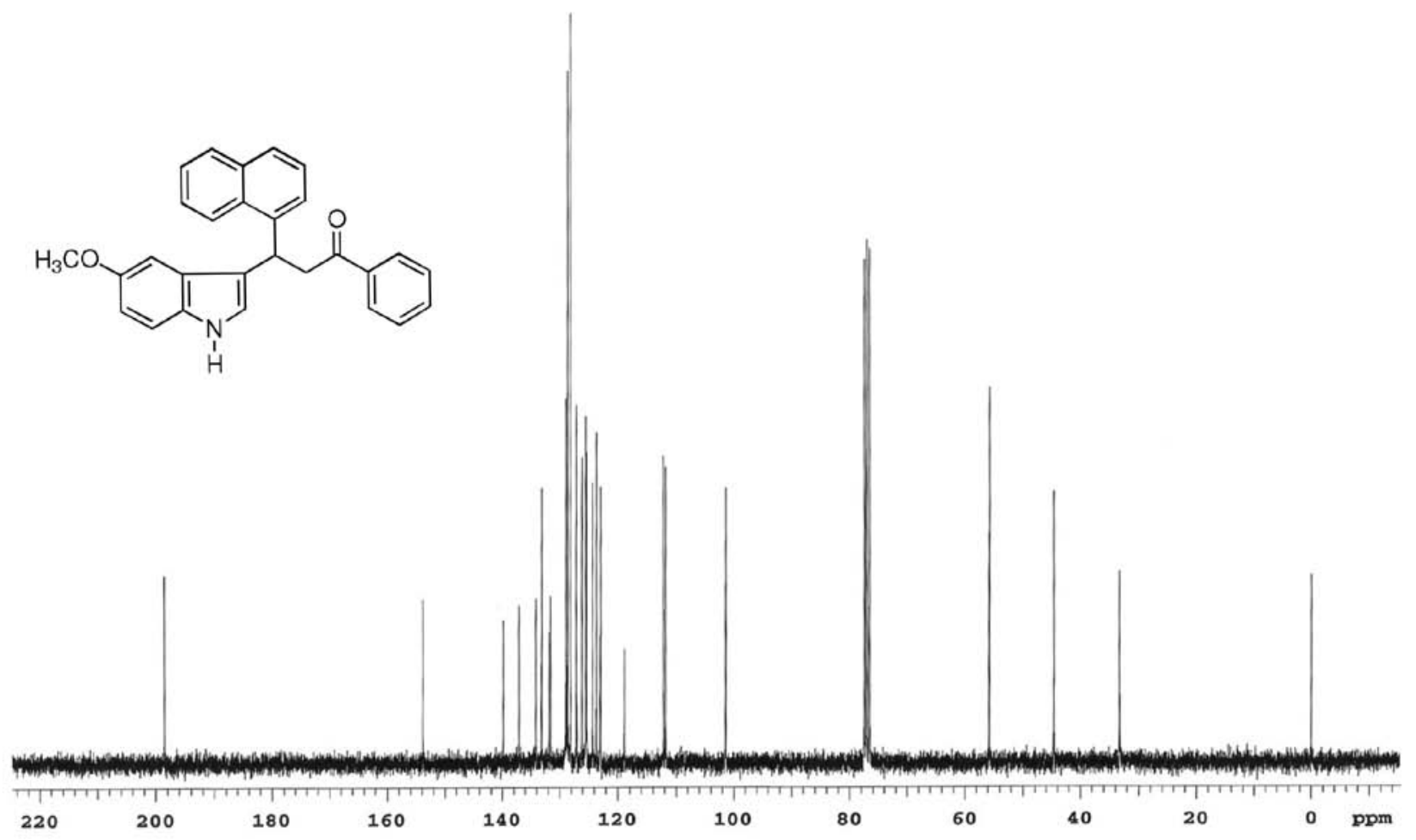

Figure S20. ${ }^{13} \mathrm{C}$ NMR $\left(\mathrm{CDCl}_{3}\right)$ of compound 3w.

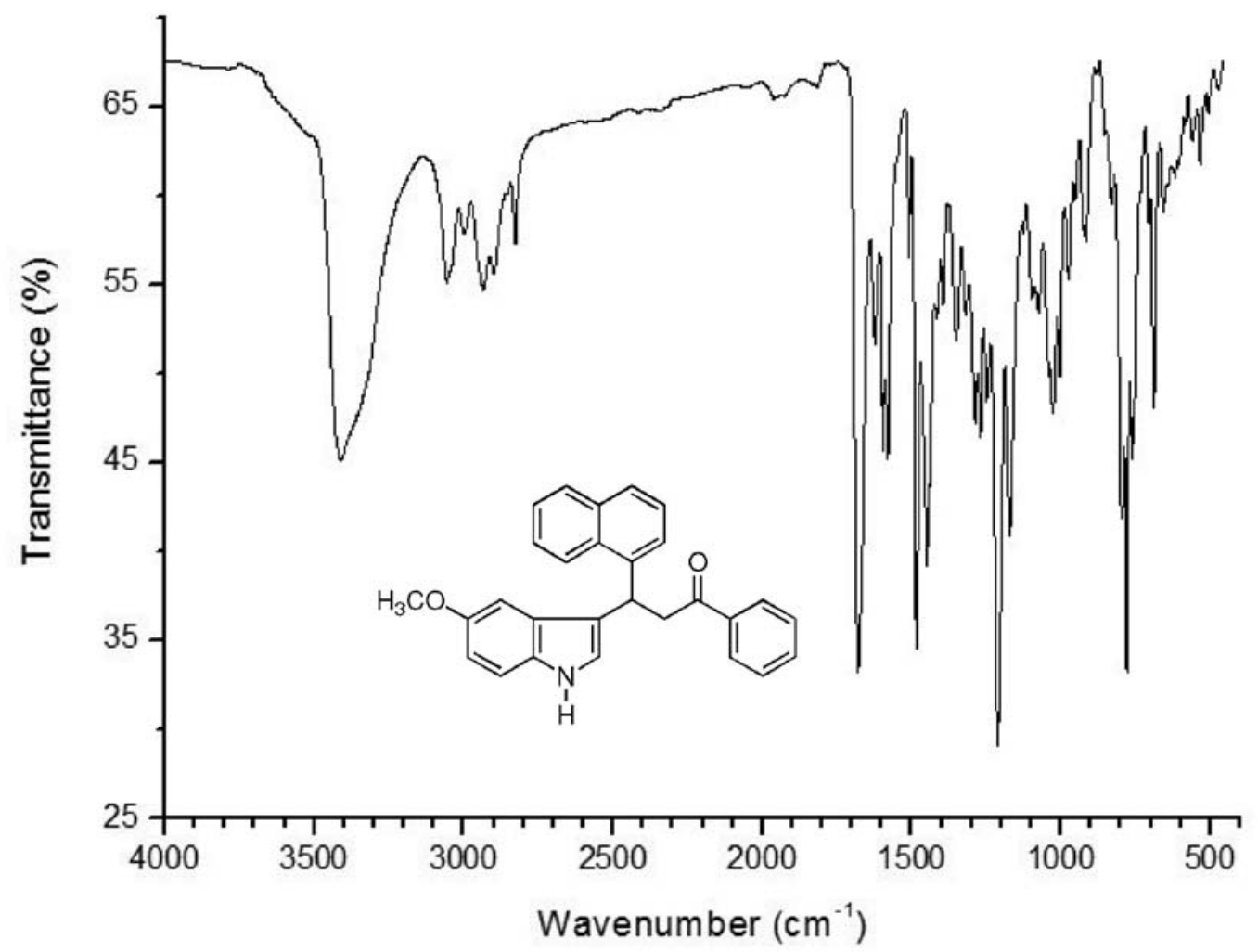

Figure S21. IR ( $\mathrm{KBr}$ ) of compound $\mathbf{3 w}$. 
<smiles>COc1ccc(C(CC(=O)c2ccccc2)c2c[nH]c3ccc(OC)cc23)cc1</smiles>

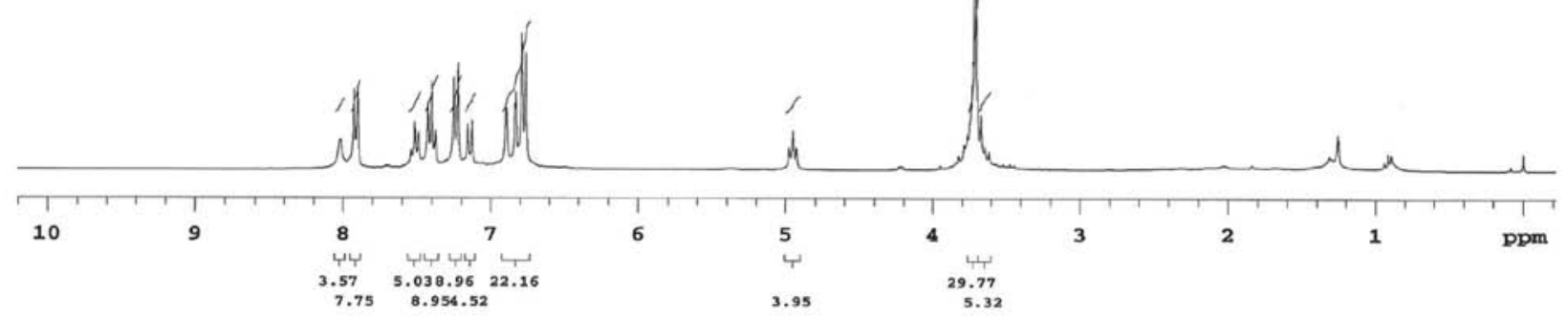

Figure S22. ${ }^{1} \mathrm{H}$ NMR $\left(\mathrm{CDCl}_{3}\right)$ of compound $\mathbf{3 x}$.<smiles>COc1ccc(C(CC(=O)c2ccccc2)c2c[nH]c3ccc(OC)cc23)cc1</smiles>

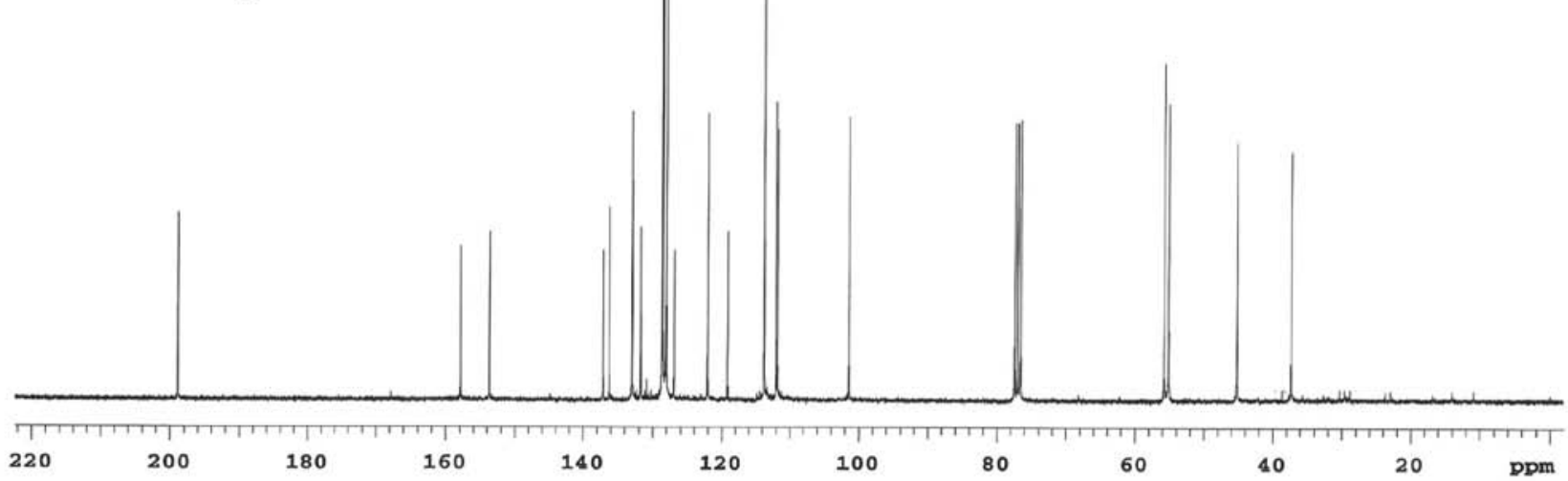

Figure S23. ${ }^{13} \mathrm{C}$ NMR $\left(\mathrm{CDCl}_{3}\right)$ of compound 3x. 
Vol. 22, No. 4, 2011

Schwalm et al.

S13

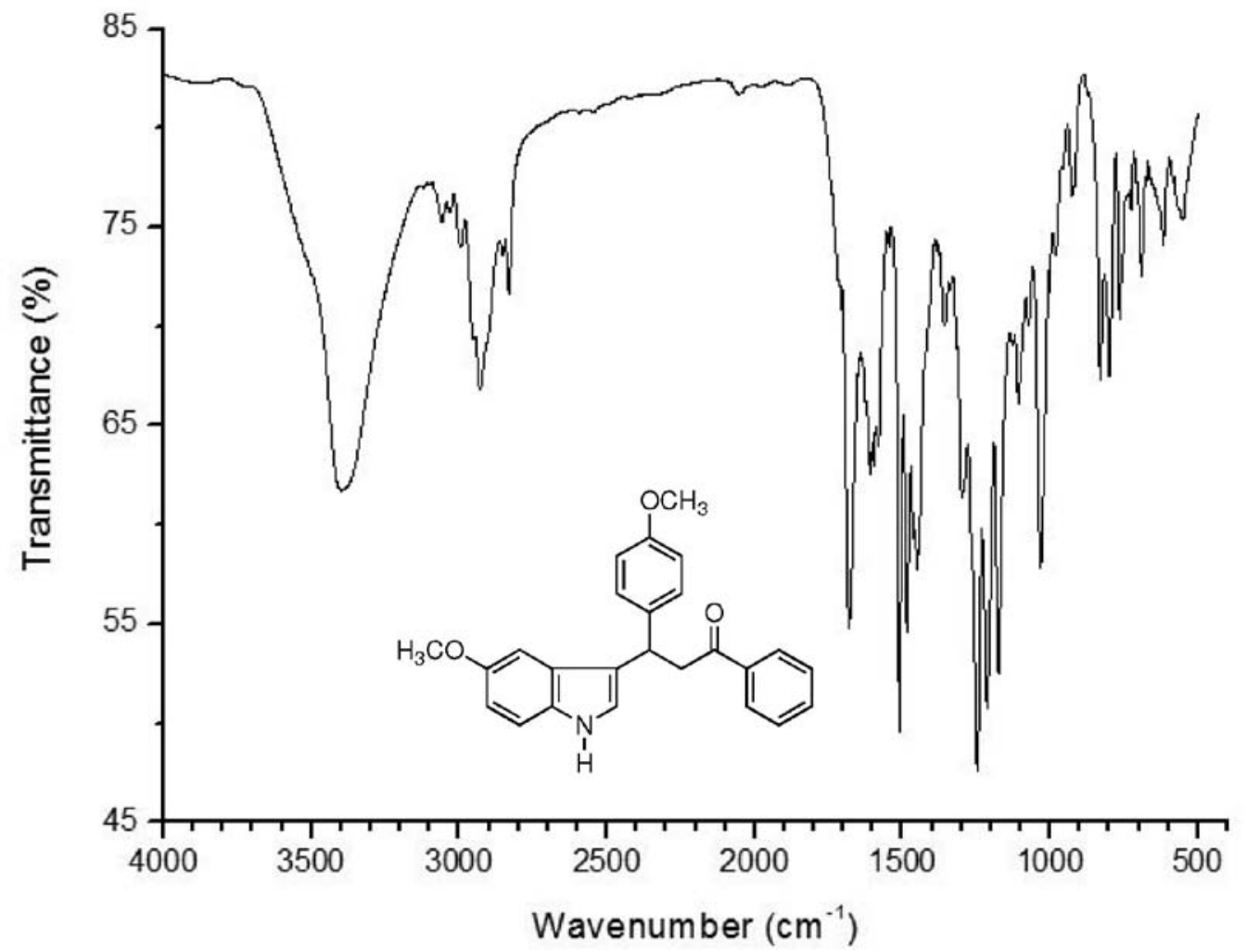

Figure S24. IR (KBr) of compound 3x.<smiles>COc1ccc2[nH]cc(C(CC(=O)c3ccccc3)c3cccs3)c2c1</smiles>

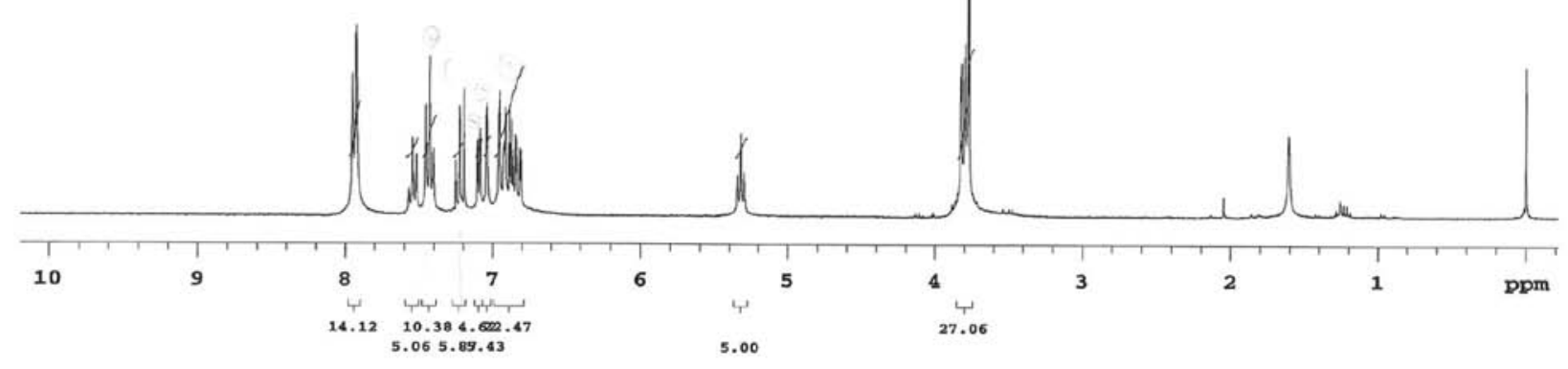

Figure S25. ${ }^{1} \mathrm{H} \mathrm{NMR}\left(\mathrm{CDCl}_{3}\right)$ of compound $\mathbf{3 y}$. 


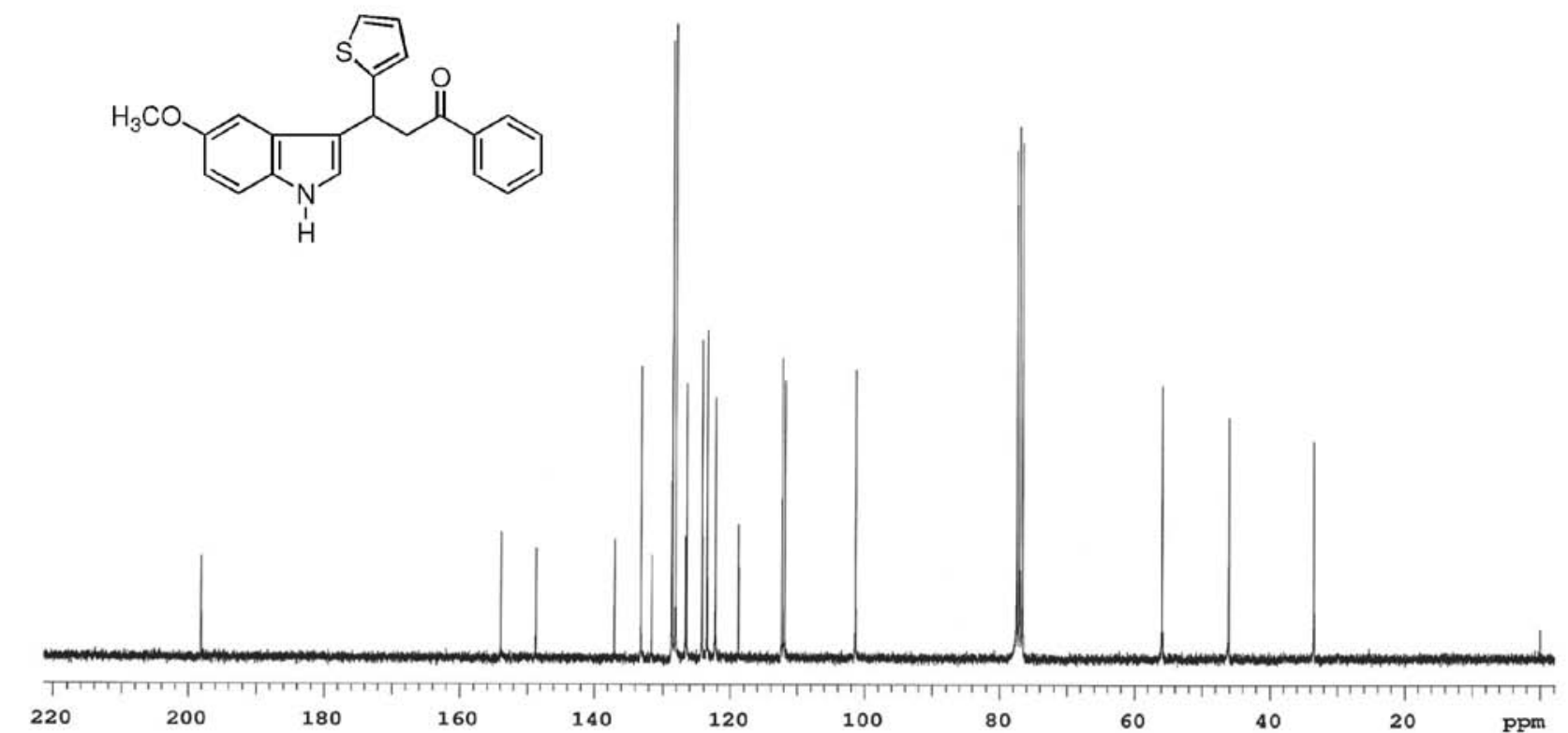

Figure S26. ${ }^{13} \mathrm{C}$ NMR $\left(\mathrm{CDCl}_{3}\right)$ of compound 3y.

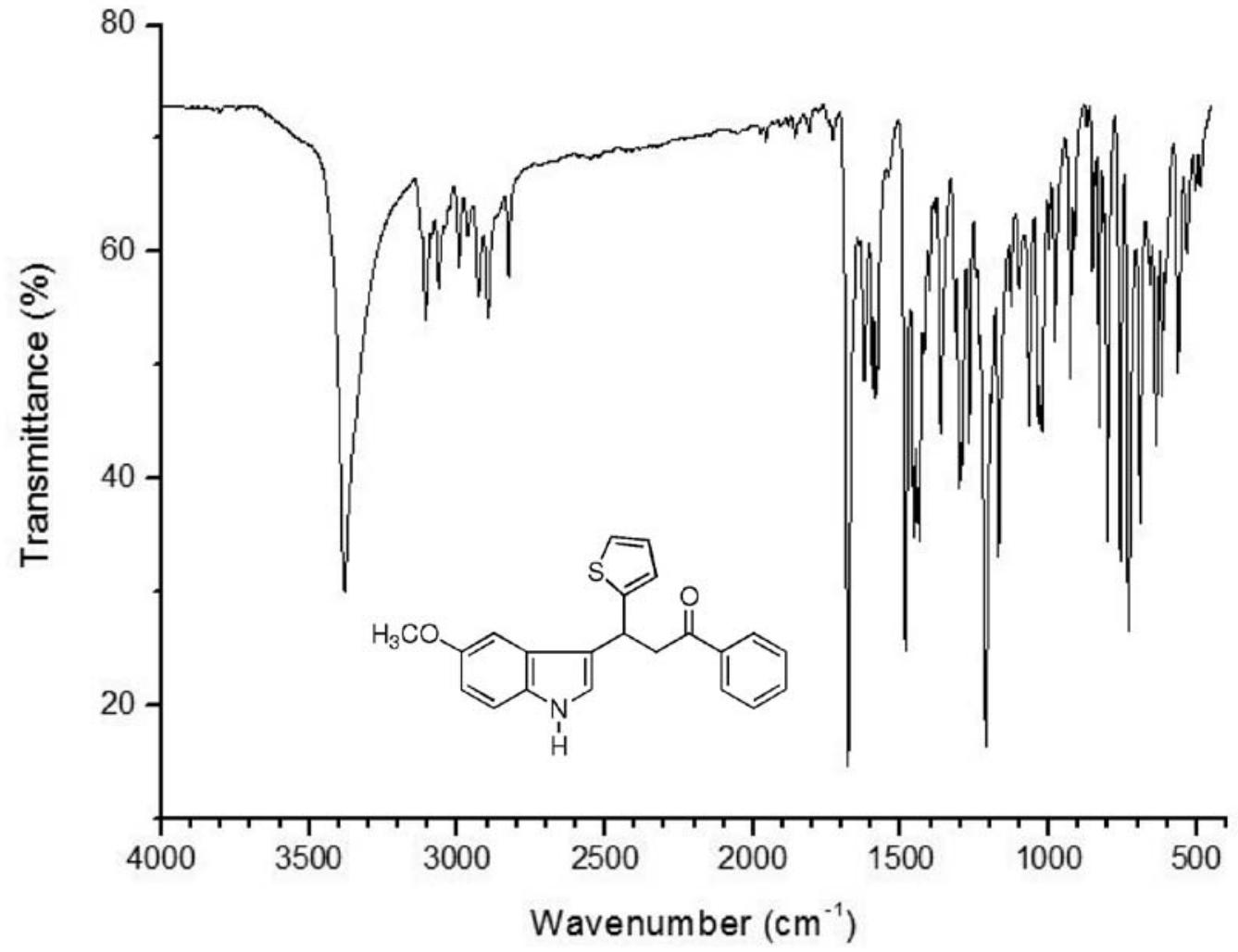

Figure S27. IR ( $\mathrm{KBr}$ ) of compound $\mathbf{3 y .}$ 
<smiles>COc1ccc(C(C[N+](=O)[O-])c2c[nH]c3ccc(OC)cc23)cc1</smiles>

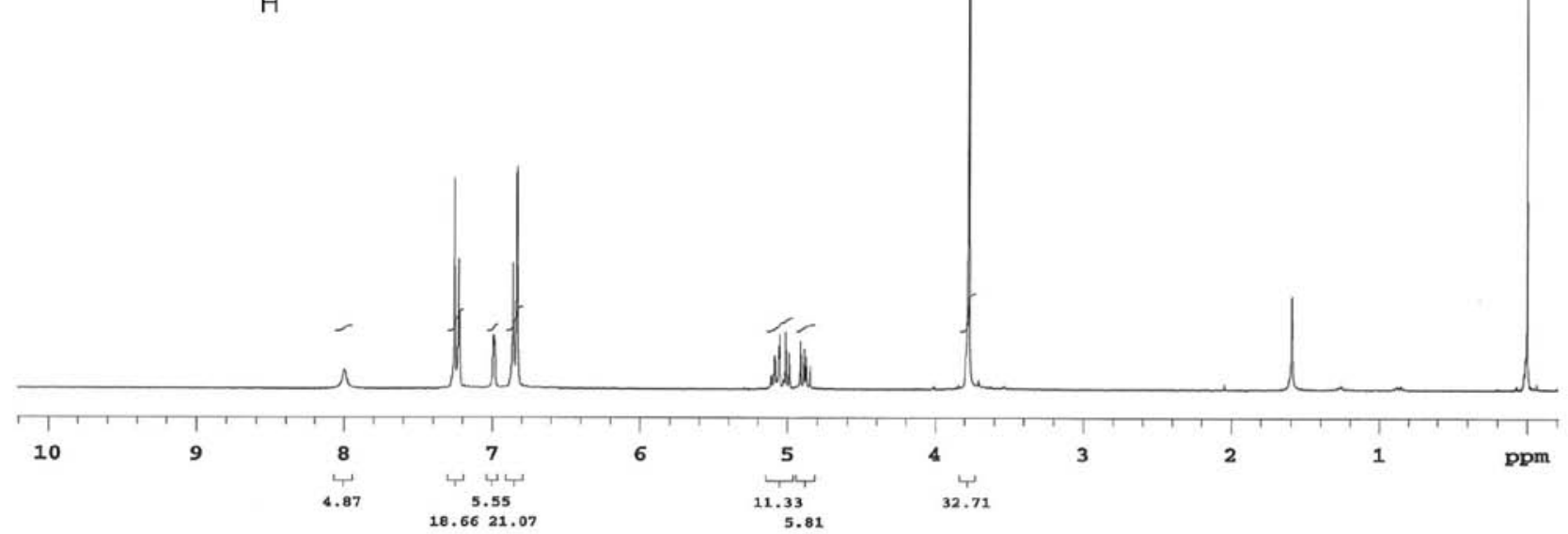

Figure S28. ${ }^{1} \mathrm{H} \mathrm{NMR}\left(\mathrm{CDCl}_{3}\right)$ of compound $\mathbf{5 i}$.

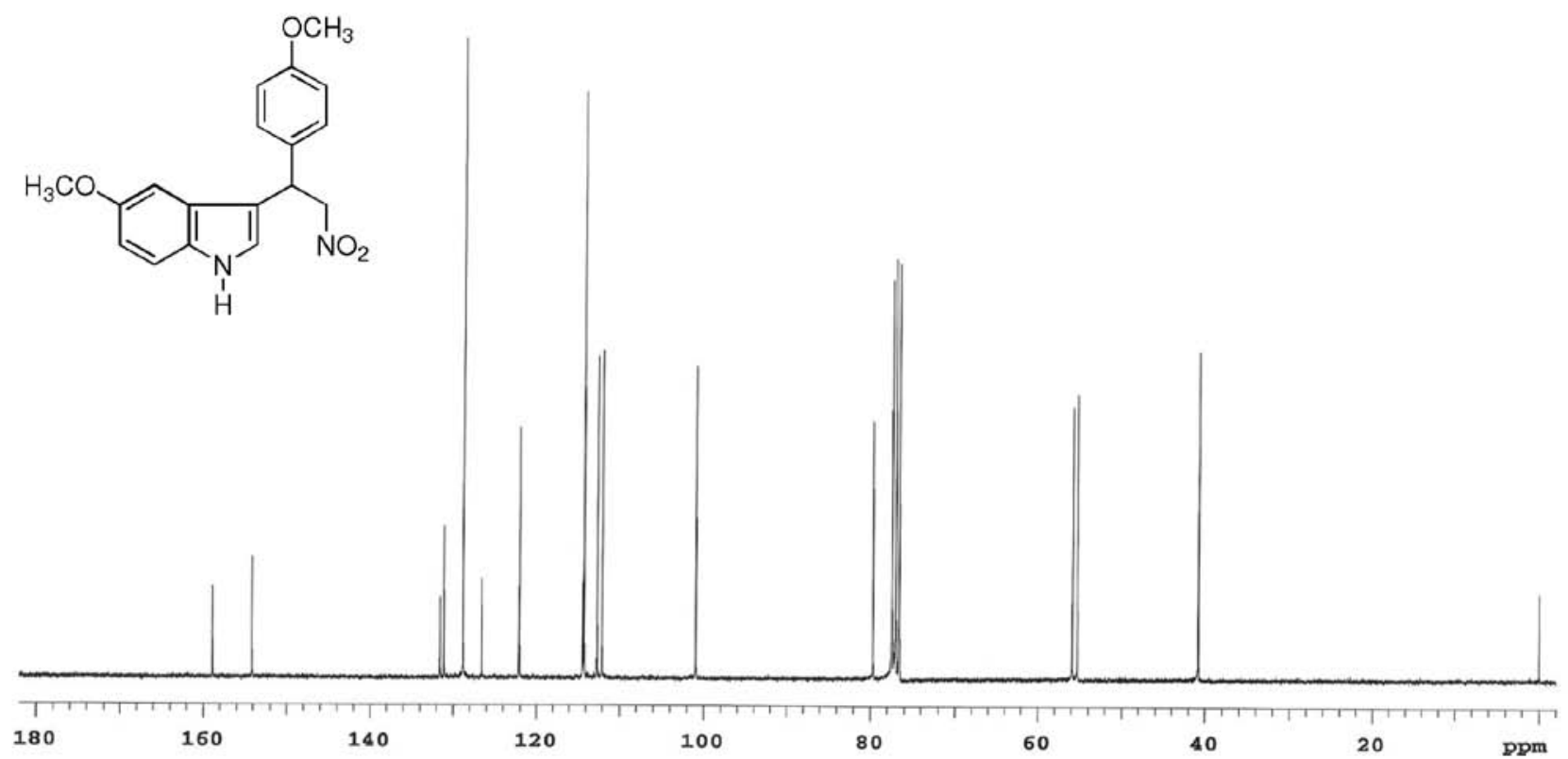

Figure S29. ${ }^{13} \mathrm{C} \mathrm{NMR}\left(\mathrm{CDCl}_{3}\right)$ of compound $\mathbf{5 i}$. 


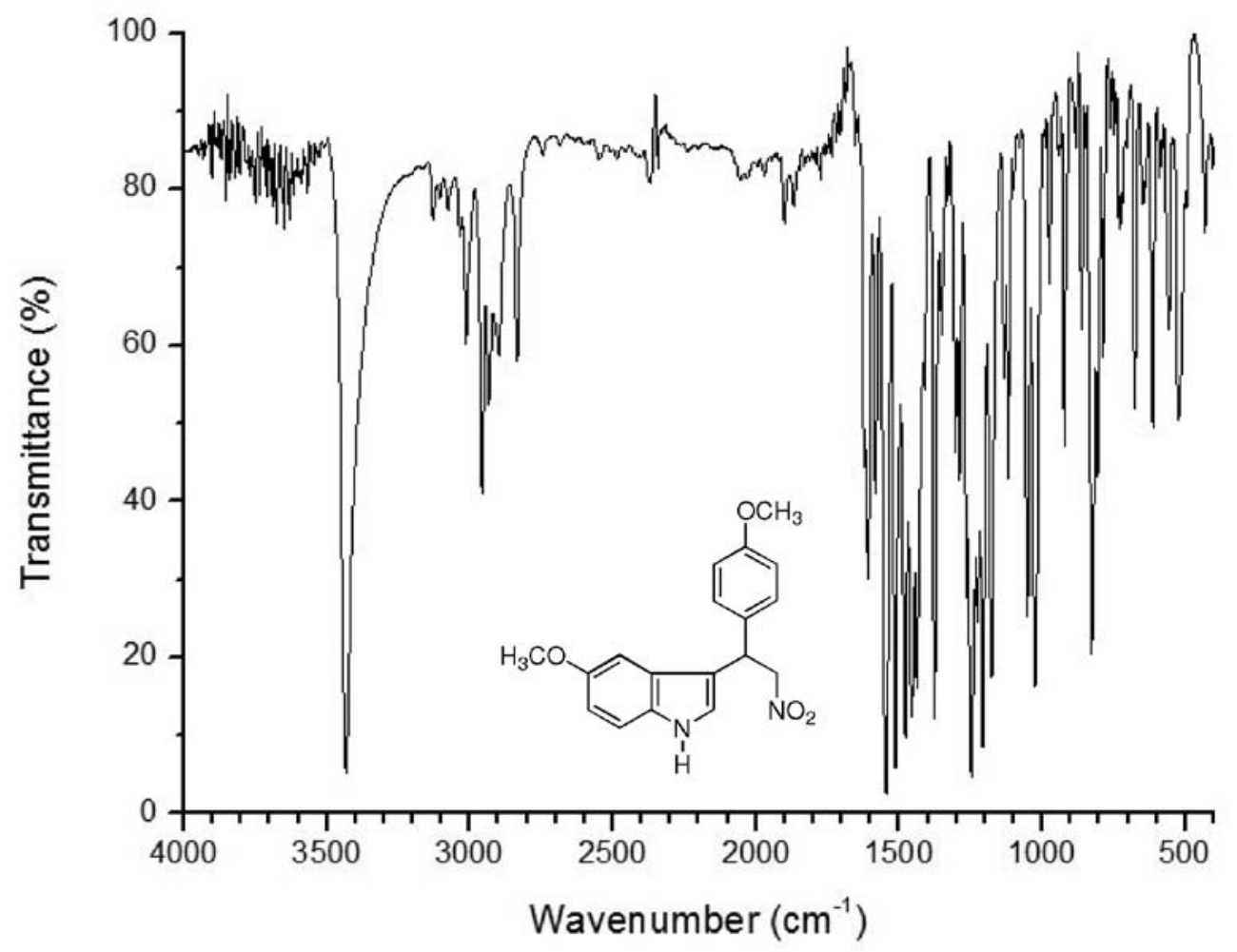

Figure S30. IR (KBr) of compound $\mathbf{5 i}$.<smiles>COc1ccc2[nH]cc(C(C[N+](=O)[O-])c3ccc(F)cc3)c2c1</smiles>

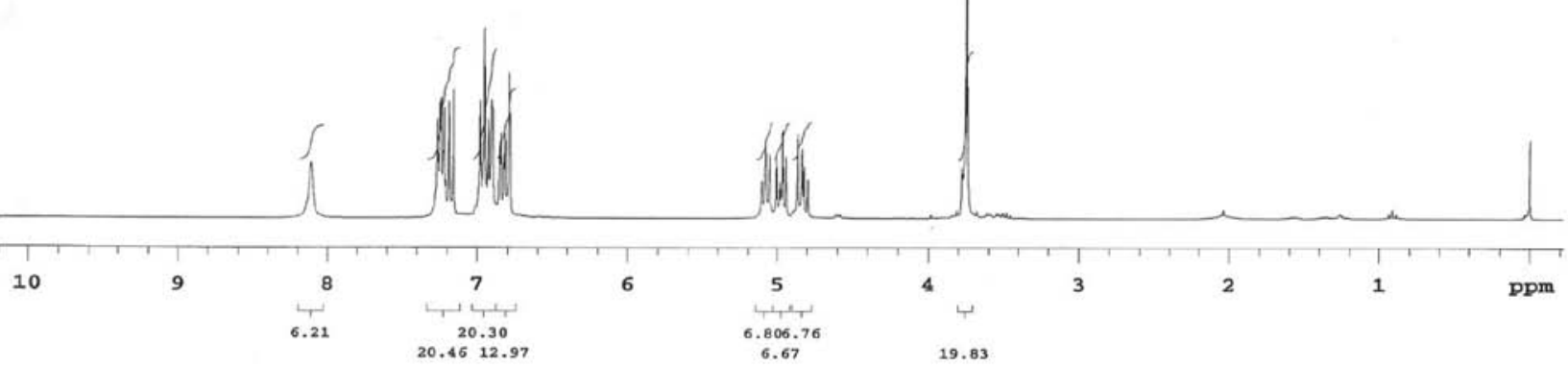

Figure S31. ${ }^{1} \mathrm{H} \mathrm{NMR}\left(\mathrm{CDCl}_{3}\right)$ of compound $\mathbf{5 j}$. 
<smiles>COc1ccc2[nH]cc(C(C[N+](=O)[O-])c3ccc(F)cc3)c2c1</smiles>

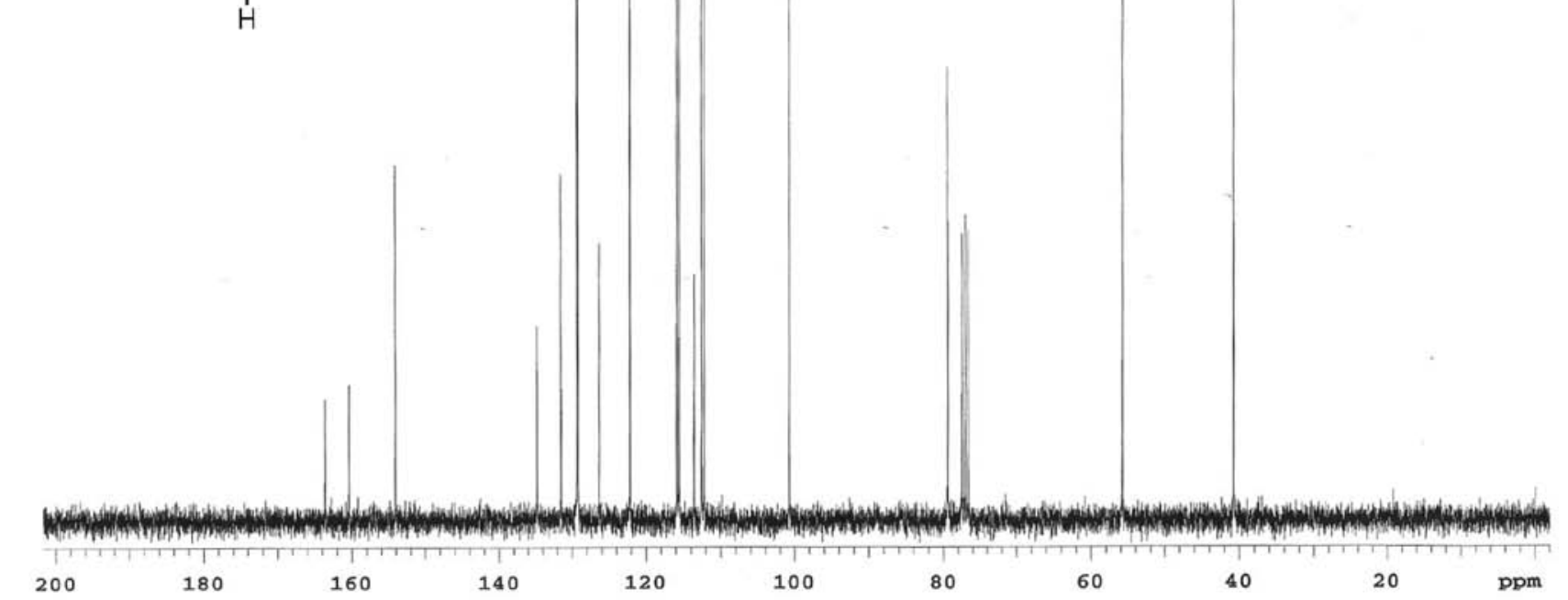

Figure S32. ${ }^{13} \mathrm{C} \mathrm{NMR}\left(\mathrm{CDCl}_{3}\right)$ of compound $\mathbf{5 j}$.

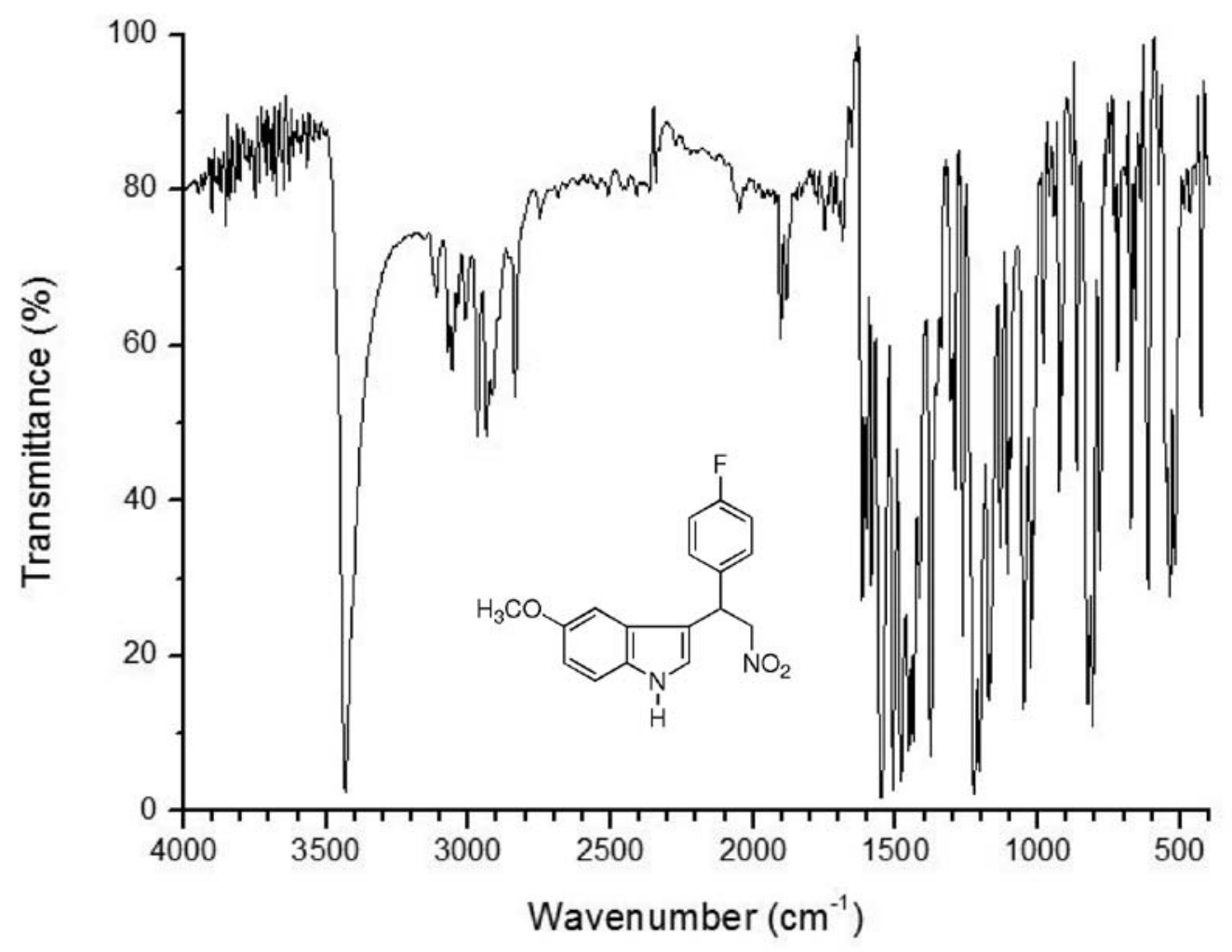

Figure S33. IR (KBr) of compound 5j. 
<smiles>COc1ccc2[nH]cc(C(C[N+](=O)[O-])c3ccc(N(C)C)cc3)c2c1</smiles>

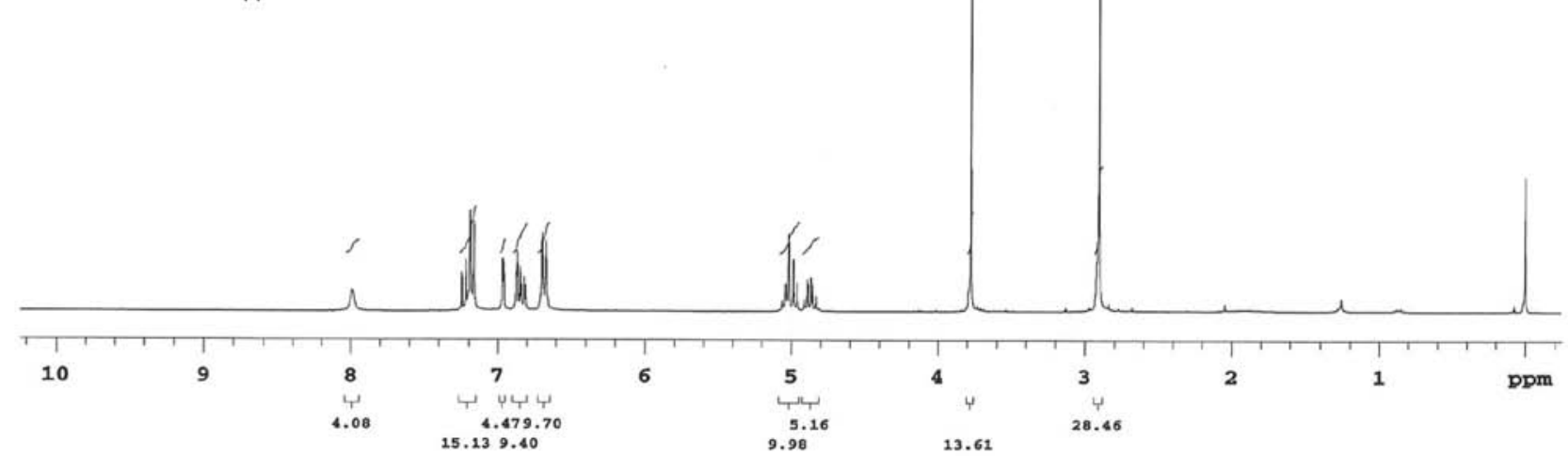

Figure S34. ${ }^{1} \mathrm{H} \mathrm{NMR}\left(\mathrm{CDCl}_{3}\right)$ of compound $\mathbf{5 k}$.<smiles>COc1ccc2[nH]cc(C(C[N+](=O)[O-])c3ccc(N(C)C)cc3)c2c1</smiles>

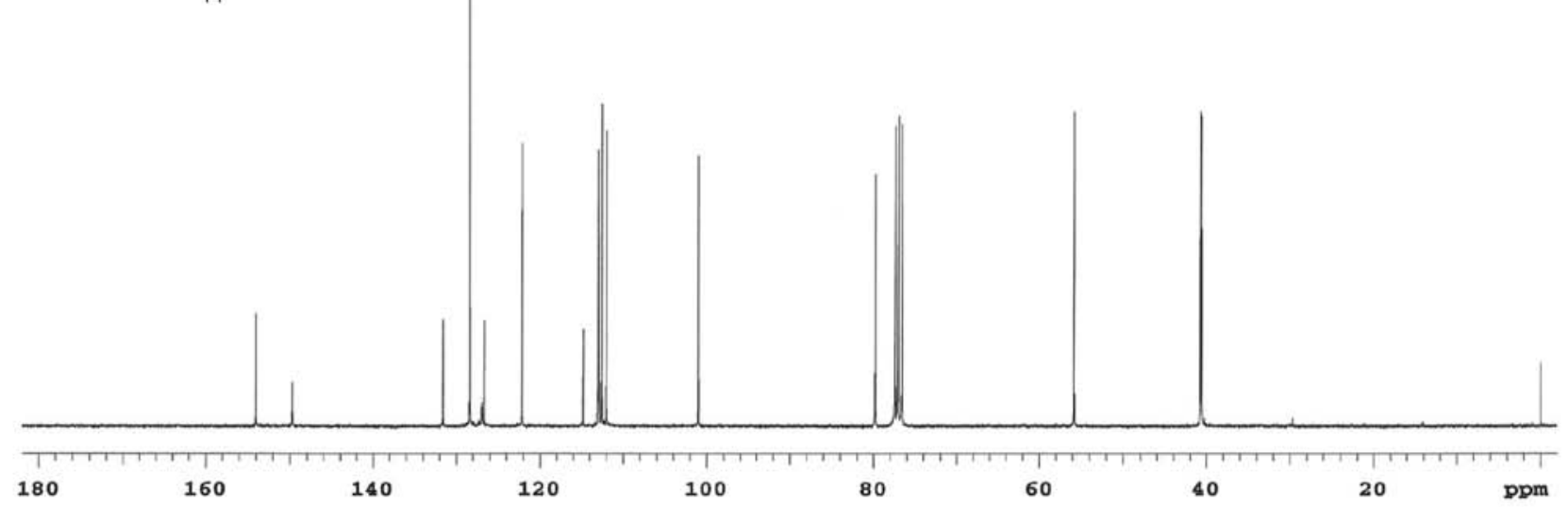

Figure S35. ${ }^{13} \mathrm{C}$ NMR $\left(\mathrm{CDCl}_{3}\right)$ of compound $\mathbf{5 k}$. 


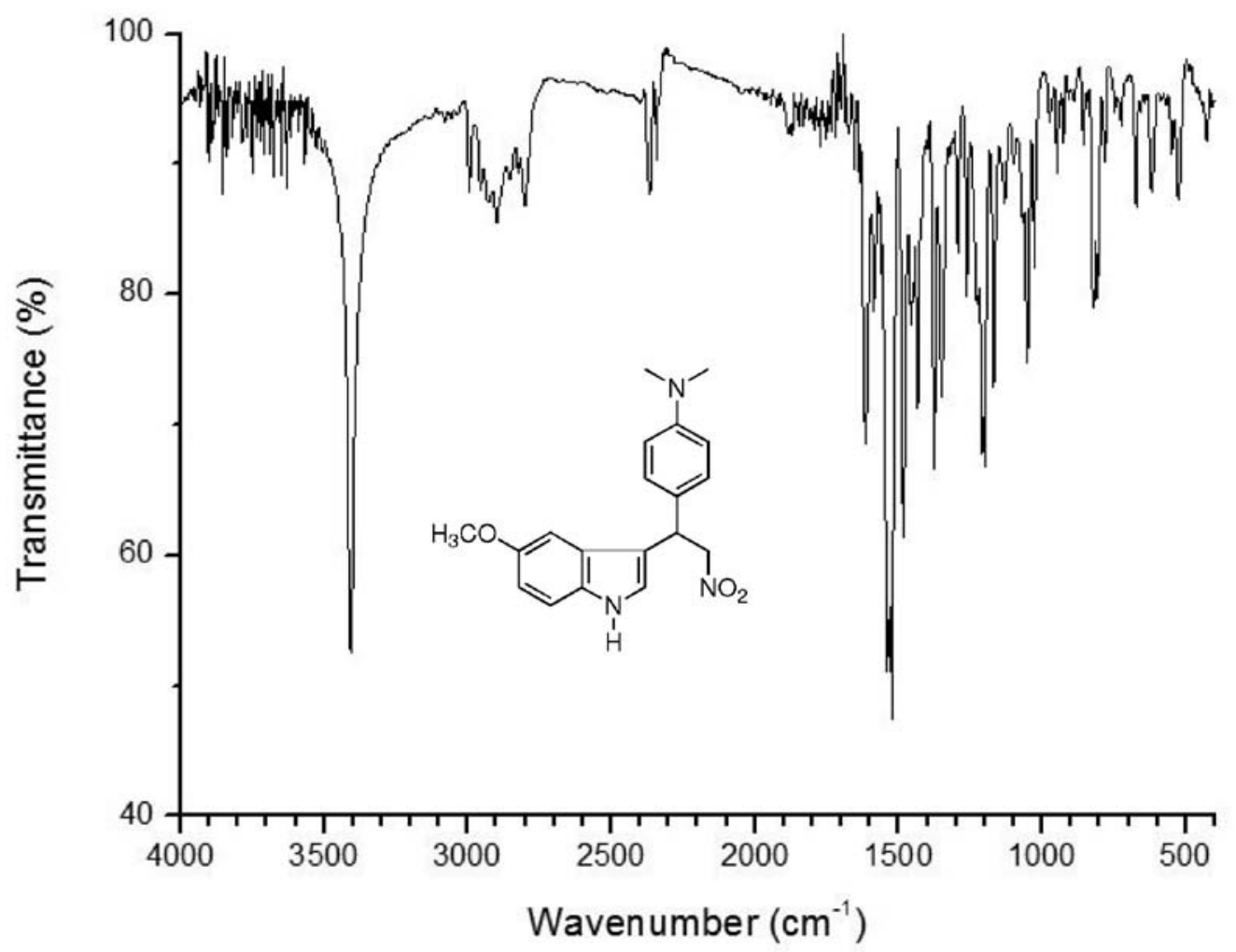

Figure S36. IR ( $\mathrm{KBr}$ ) of compound 5k.<smiles>COc1ccc2[nH]cc(C(C[N+](=O)[O-])c3ccc(OC)c(OC)c3)c2c1</smiles>

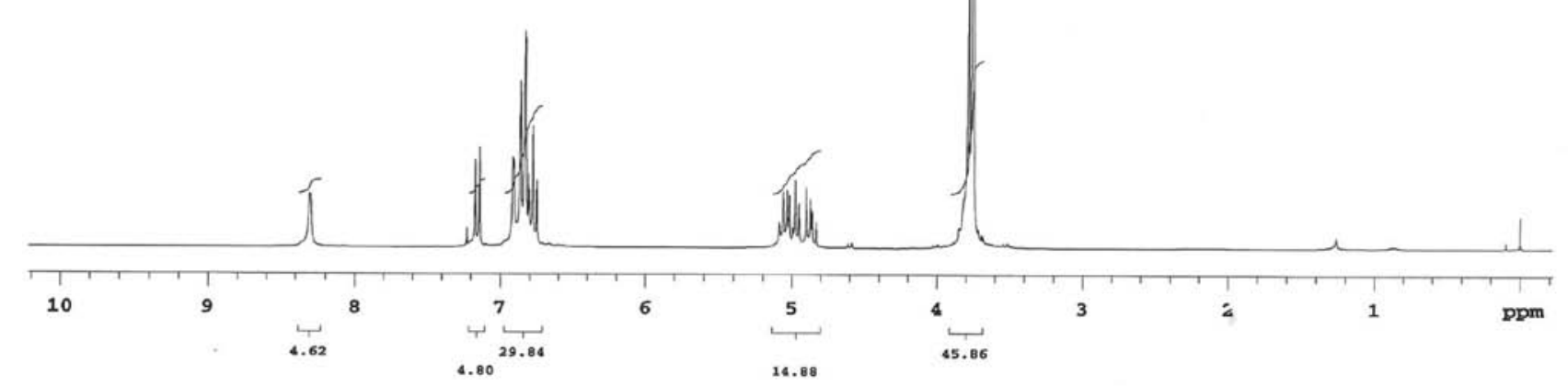

Figure S37. ${ }^{1} \mathrm{H} \mathrm{NMR}\left(\mathrm{CDCl}_{3}\right)$ of compound 5 l. 
<smiles>COc1ccc2[nH]cc(C(C[N+](=O)[O-])c3ccc(OC)c(OC)c3)c2c1</smiles>

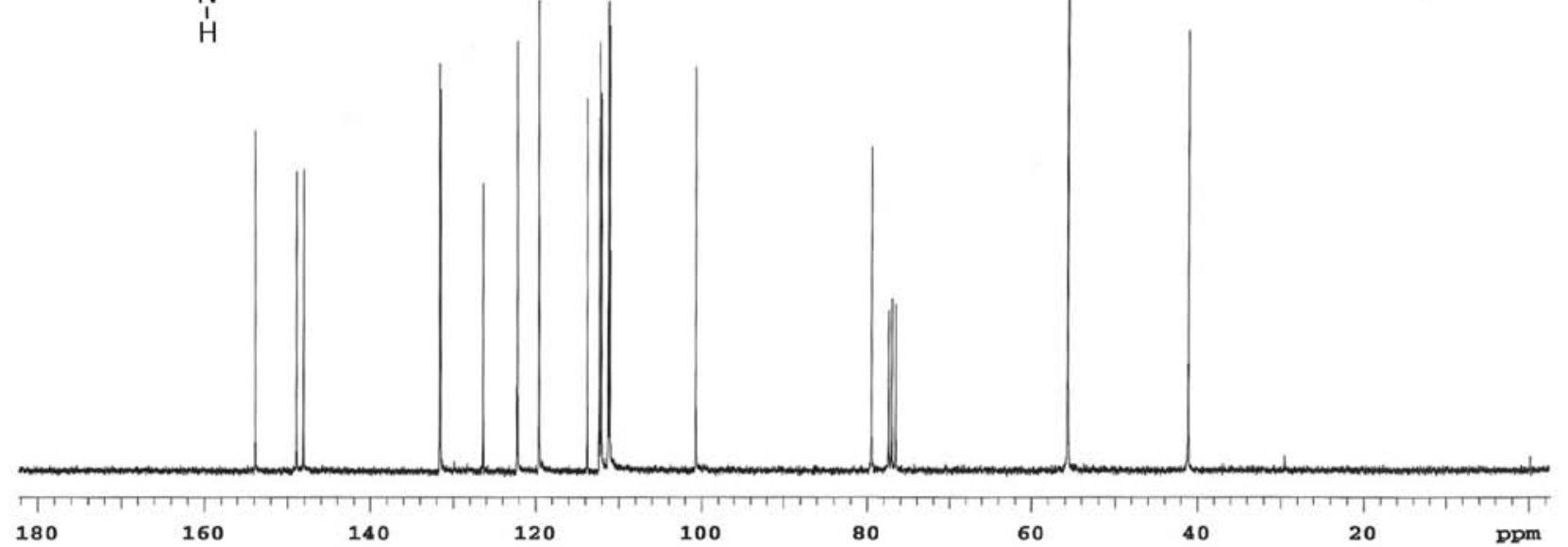

Figure S38. ${ }^{13} \mathrm{C}$ NMR $\left(\mathrm{CDCl}_{3}\right)$ of compound $\mathbf{5 l}$.

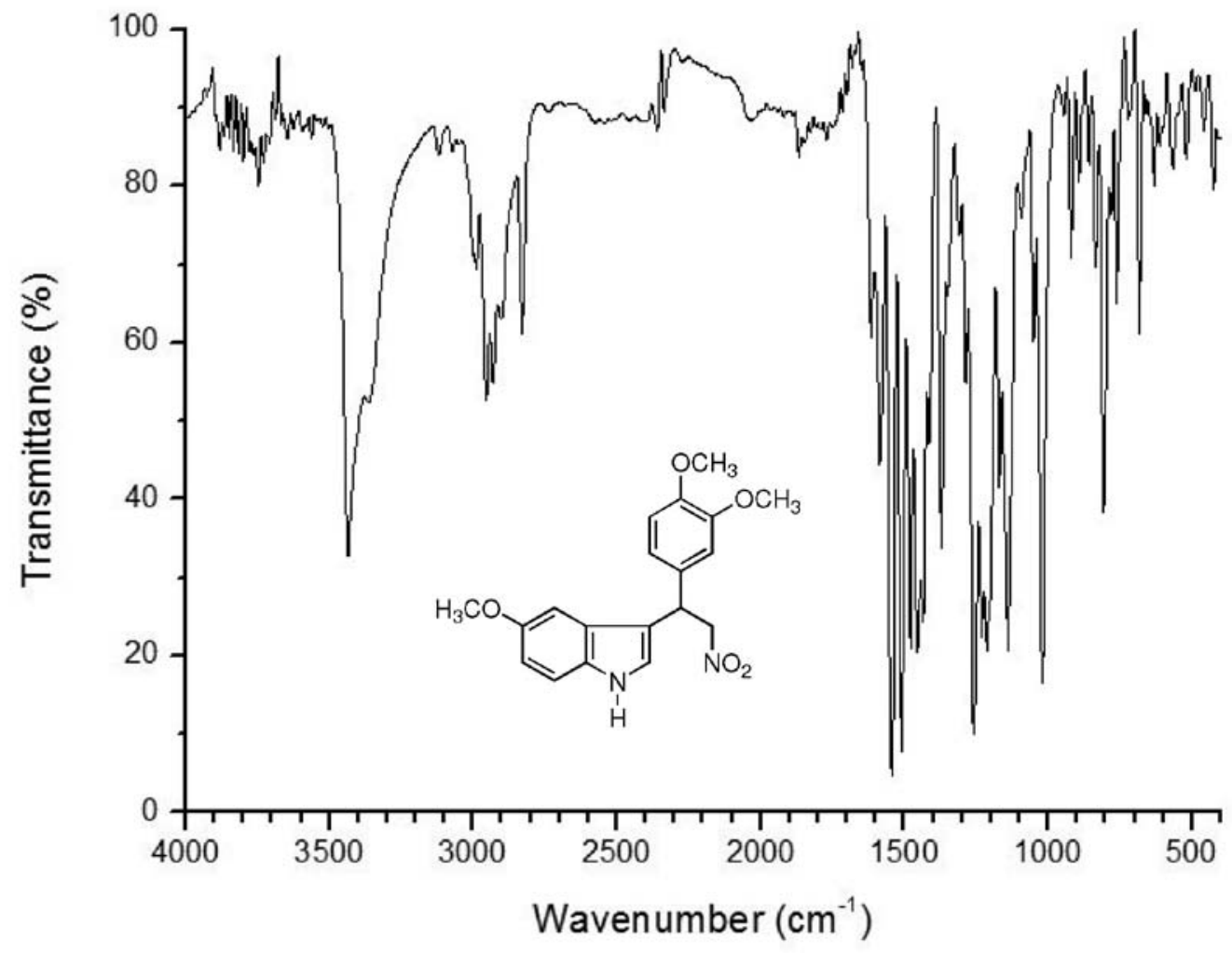

Figure S39. IR (KBr) of compound 5 I. 
<smiles>COc1ccc2[nH]cc(C(C[N+](=O)[O-])c3cccc4ccccc34)c2c1</smiles>

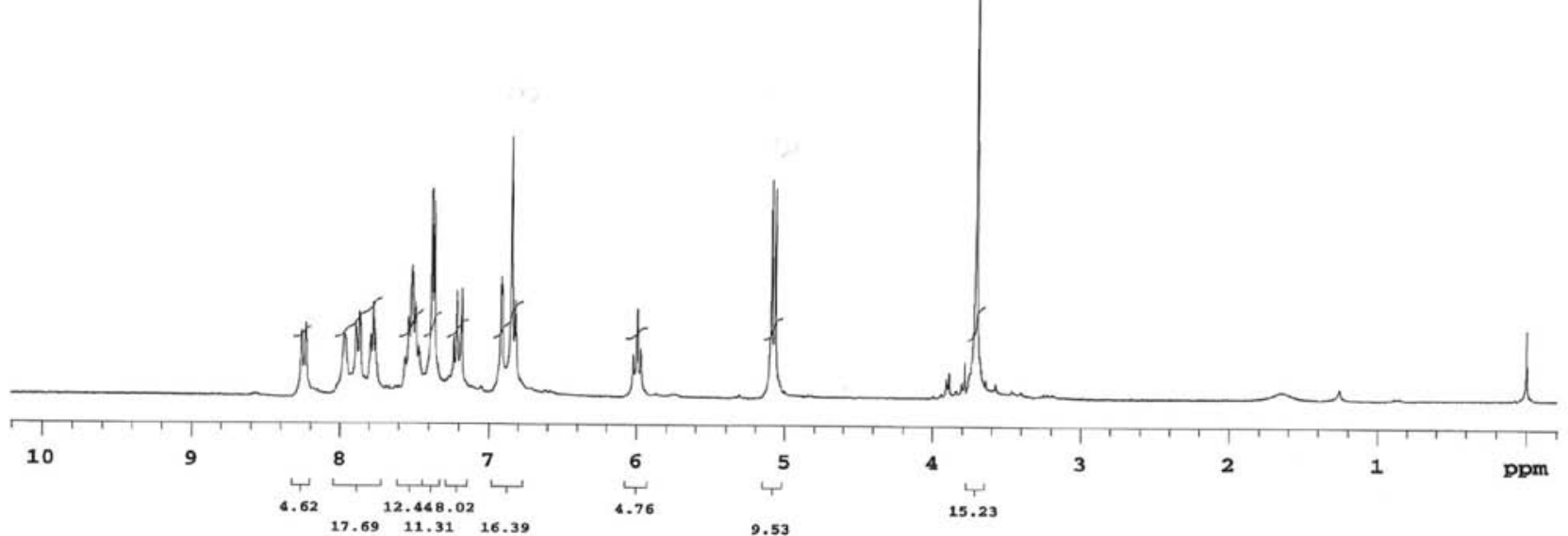

Figure S40. ${ }^{1} \mathrm{H}$ NMR $\left(\mathrm{CDCl}_{3}\right)$ of compound $\mathbf{5 m}$.<smiles>COc1ccc2[nH]cc(C(C[N+](=O)[O-])c3cccc4ccccc34)c2c1</smiles>

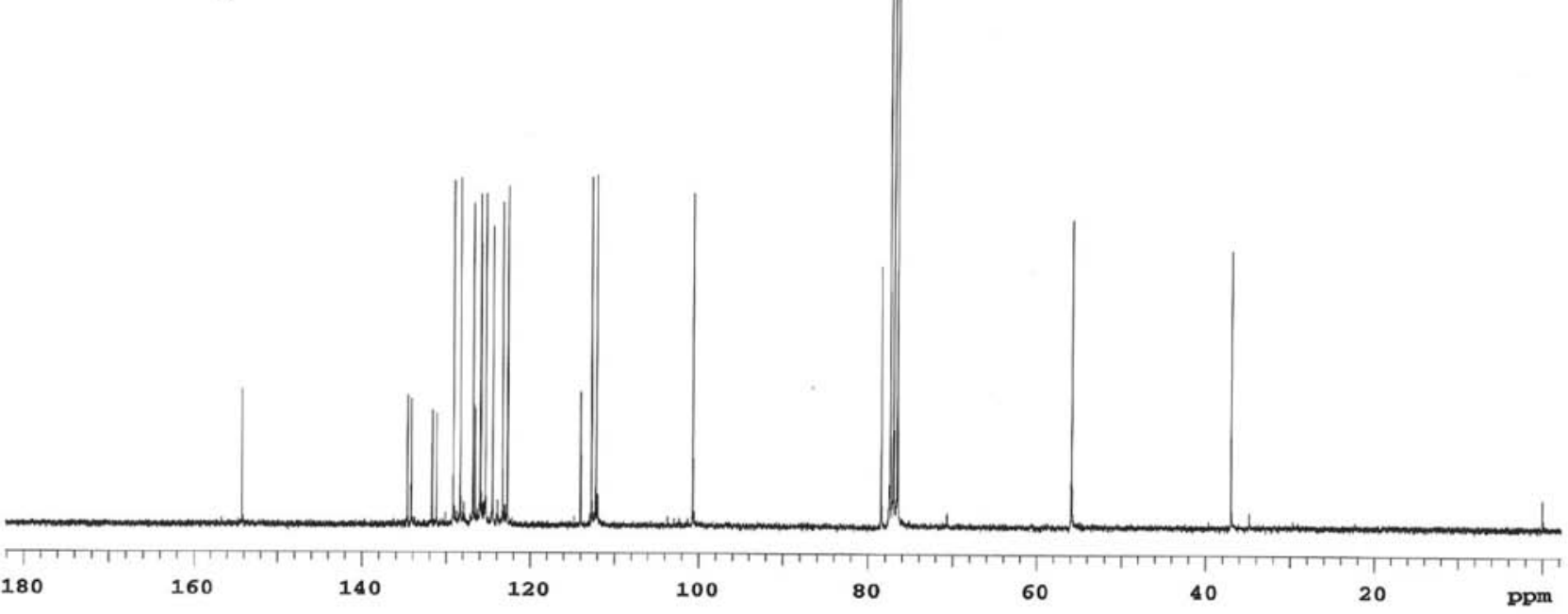

Figure S41. ${ }^{13} \mathrm{C} \mathrm{NMR}\left(\mathrm{CDCl}_{3}\right)$ of compound $\mathbf{5 m}$. 


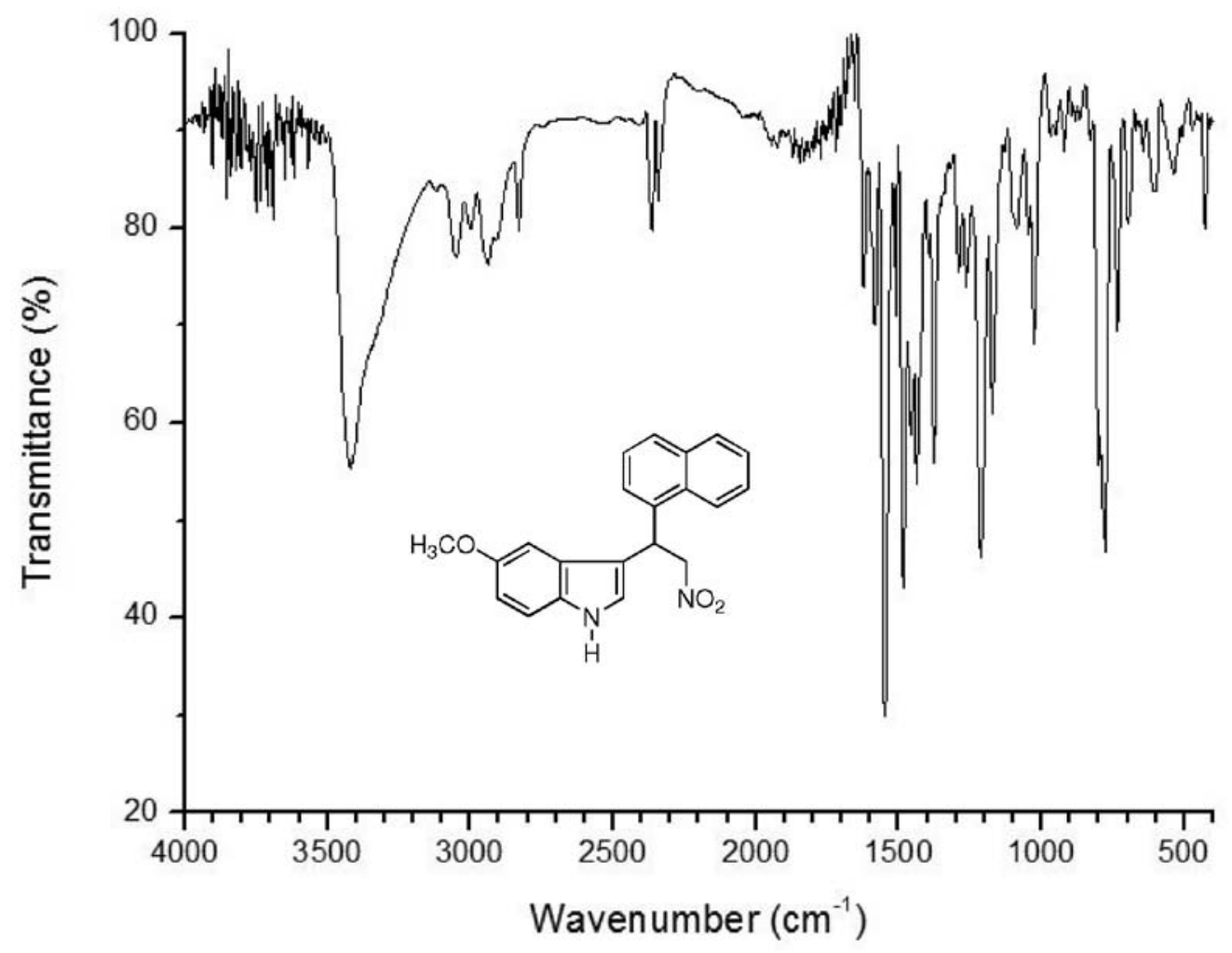

Figure S42. IR (KBr) of compound $\mathbf{5 m}$.<smiles>COc1ccc2[nH]cc(C(C[N+](=O)[O-])c3cccs3)c2c1</smiles>

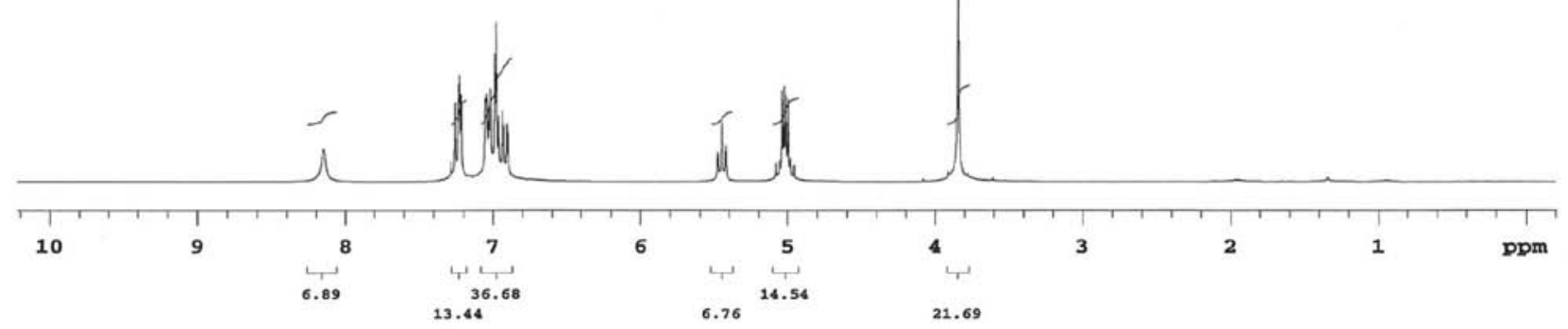

Figure S43. ${ }^{1} \mathrm{H}$ NMR $\left(\mathrm{CDCl}_{3}\right)$ of compound $\mathbf{5 n}$. 


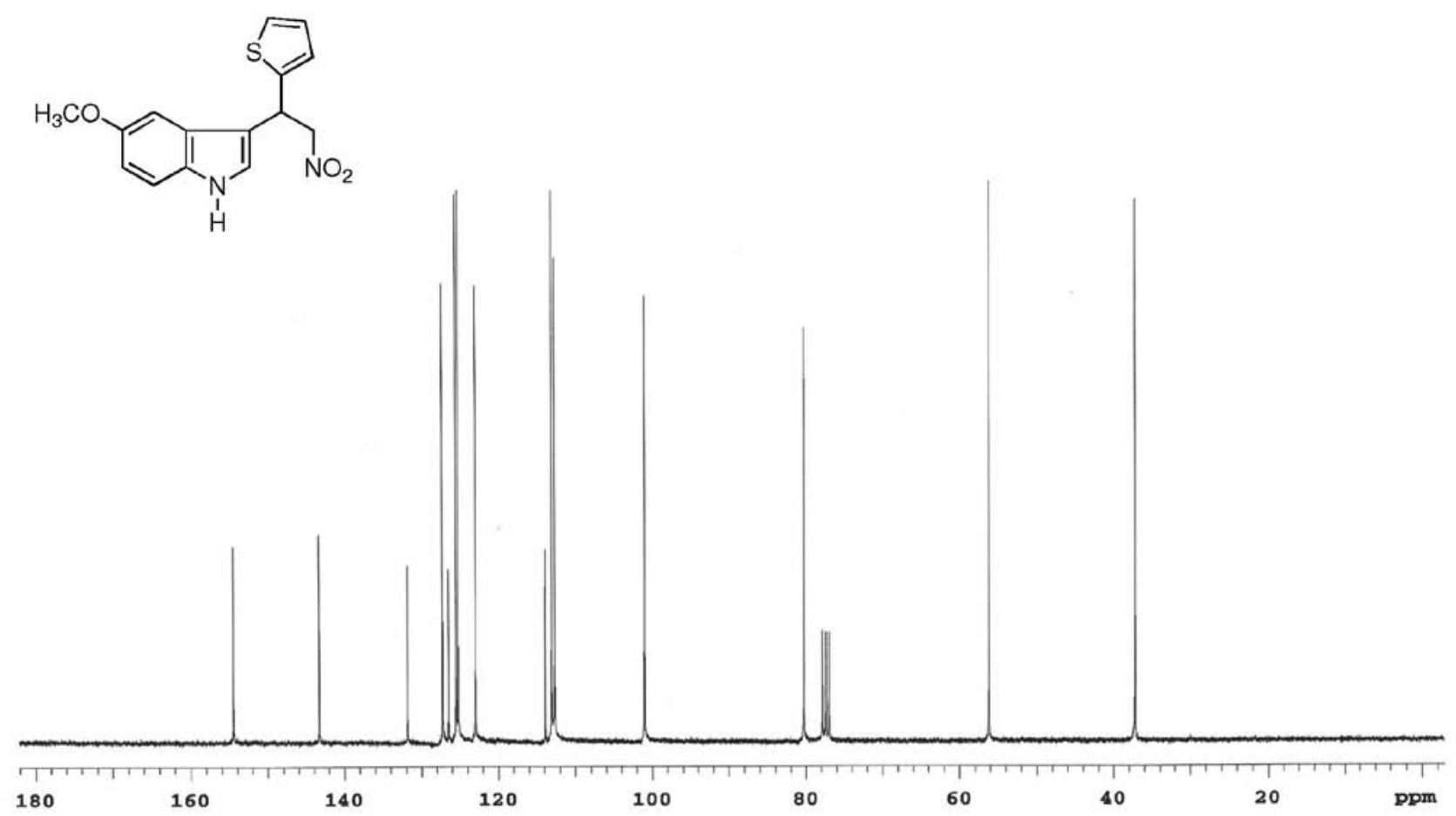

Figure S44. ${ }^{13} \mathrm{C}$ NMR $\left(\mathrm{CDCl}_{3}\right)$ of compound $\mathbf{5 n}$.

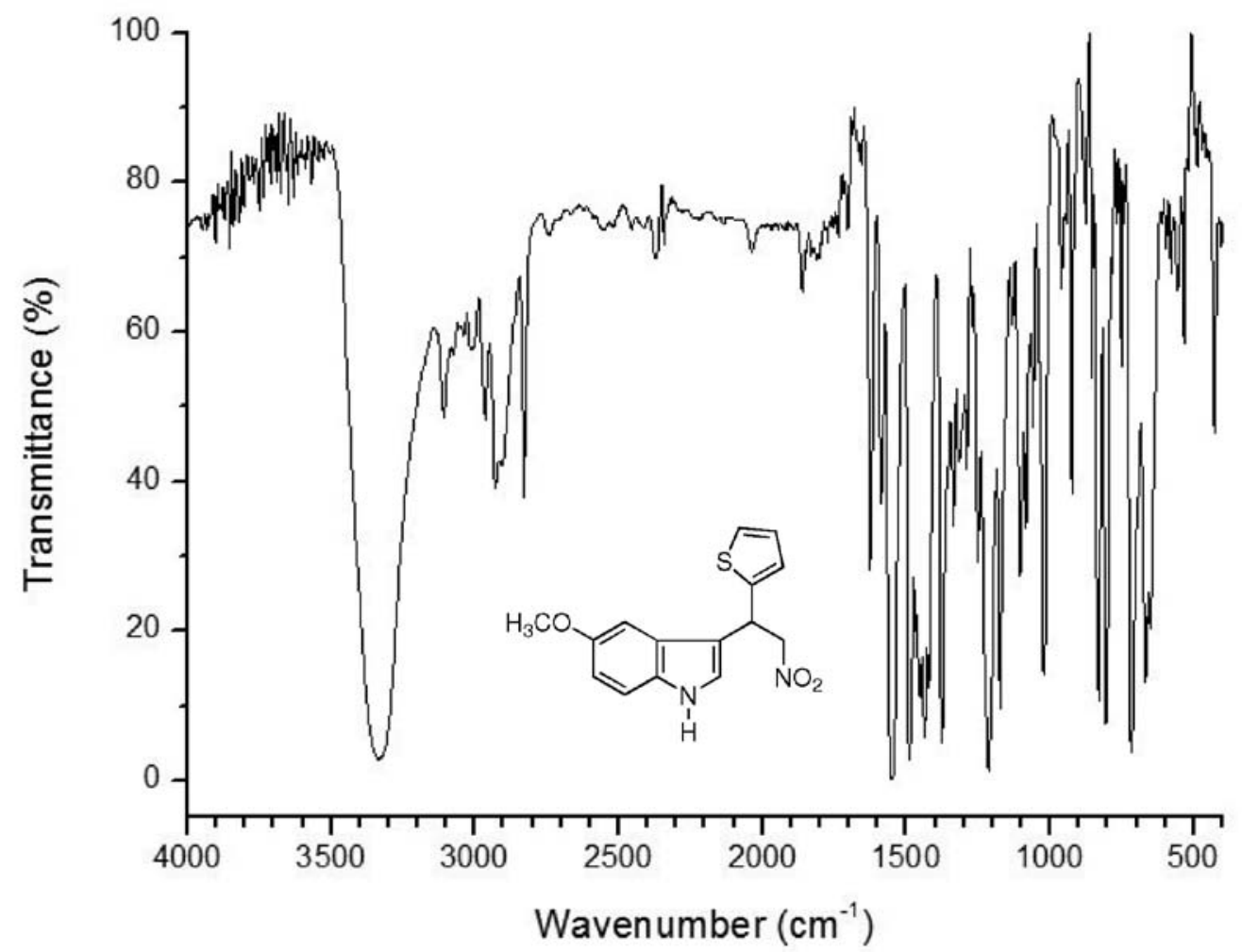

Figure S45. IR (KBr) of compound $\mathbf{5 n}$. 
<smiles>Cc1[nH]c2ccccc2c1C(C[N+](=O)[O-])c1cccc2ccccc12</smiles>

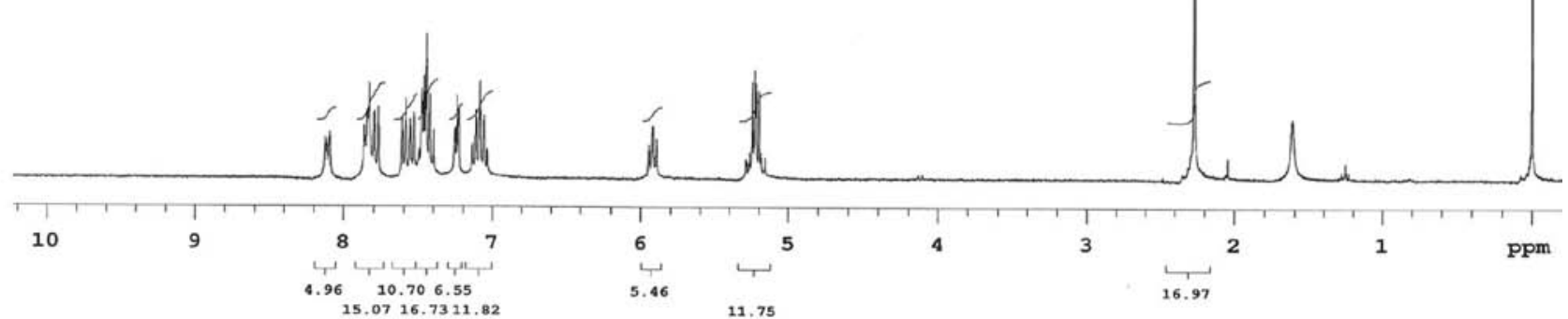

Figure S46. ${ }^{1} \mathrm{H} \mathrm{NMR}\left(\mathrm{CDCl}_{3}\right)$ of compound 5p.<smiles>Cc1[nH]c2ccccc2c1C(C[N+](=O)[O-])c1cccc2ccccc12</smiles>

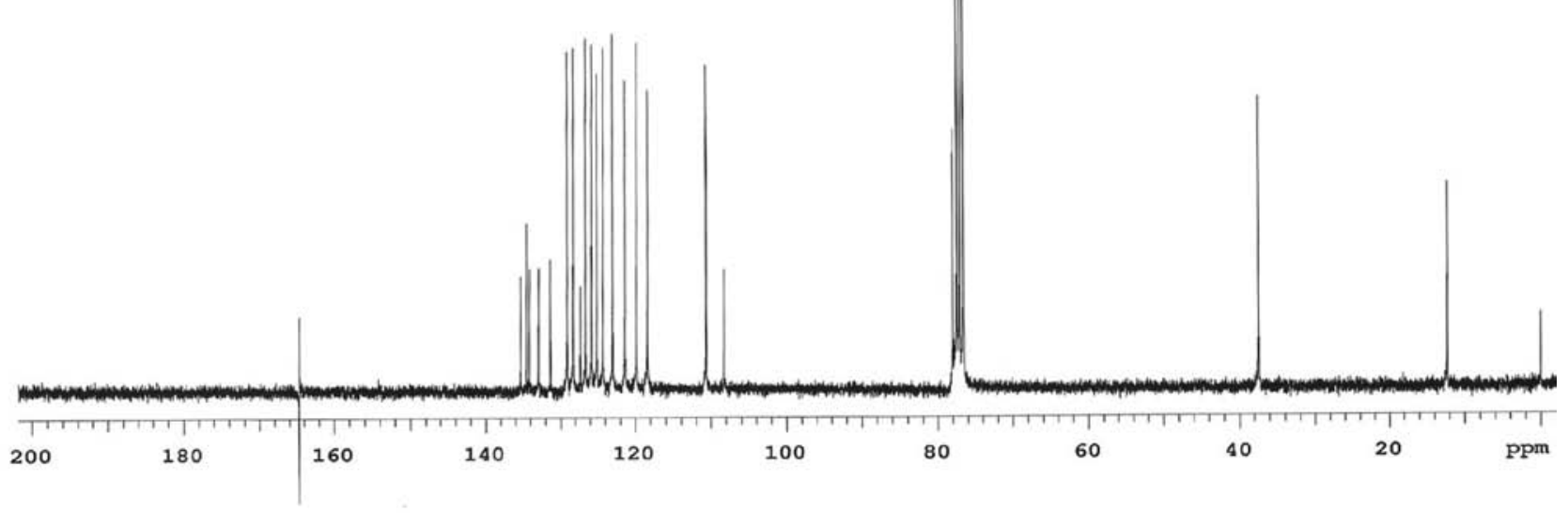

Figure S47. ${ }^{13} \mathrm{C} \mathrm{NMR}\left(\mathrm{CDCl}_{3}\right)$ of compound 5p. 


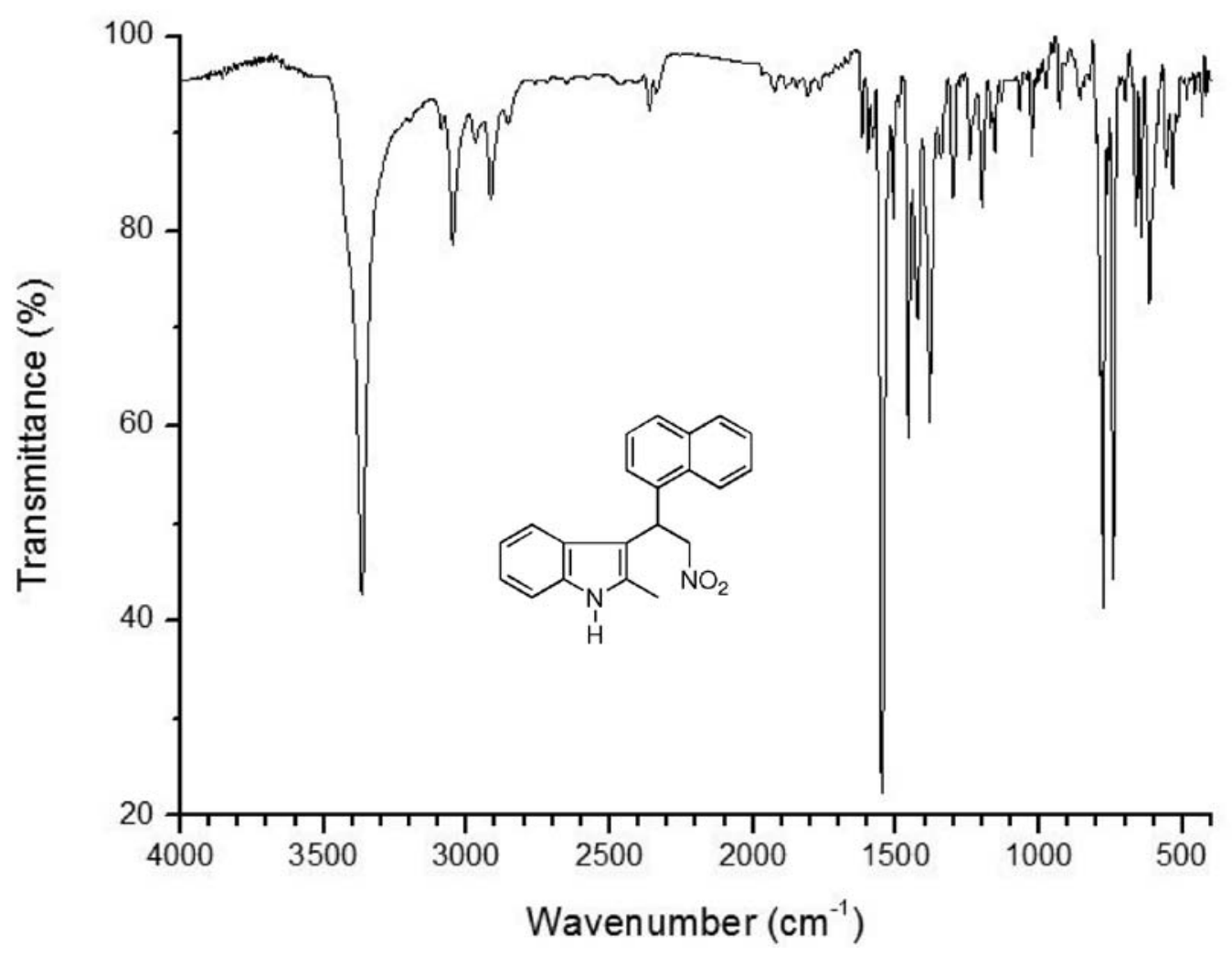

Figure S48. IR (KBr) of compound 5p.<smiles>Cc1[nH]c2ccccc2c1C(C[N+](=O)[O-])c1cccs1</smiles>

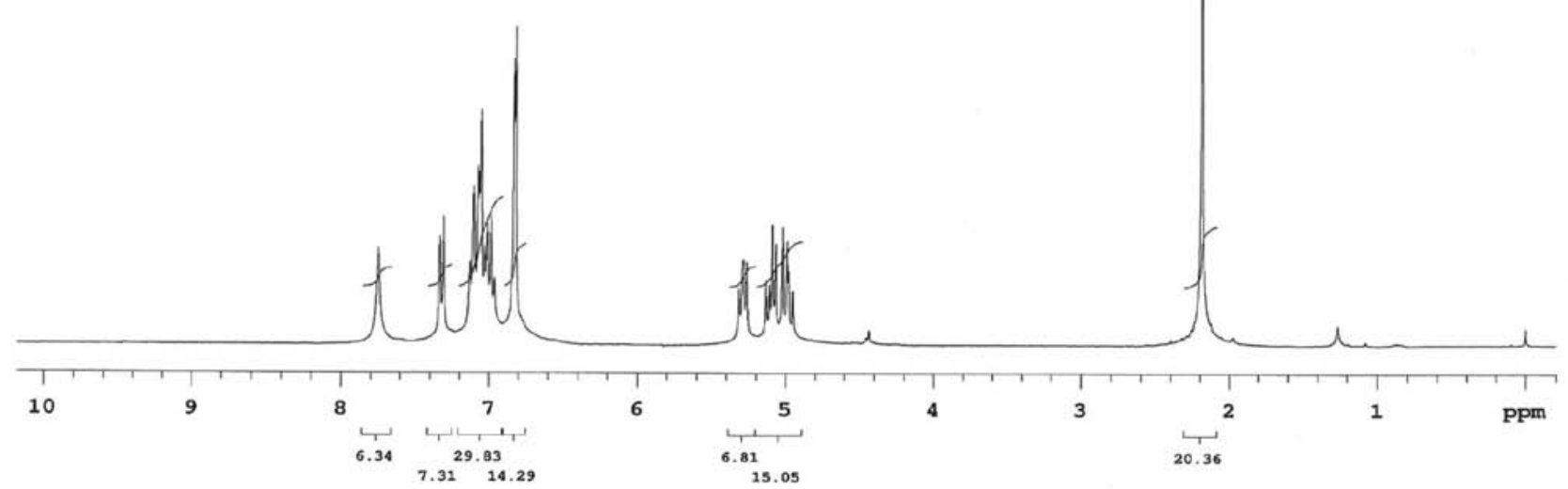

Figure S49. ${ }^{1} \mathrm{H} \mathrm{NMR}\left(\mathrm{CDCl}_{3}\right)$ of compound $\mathbf{5 q}$. 


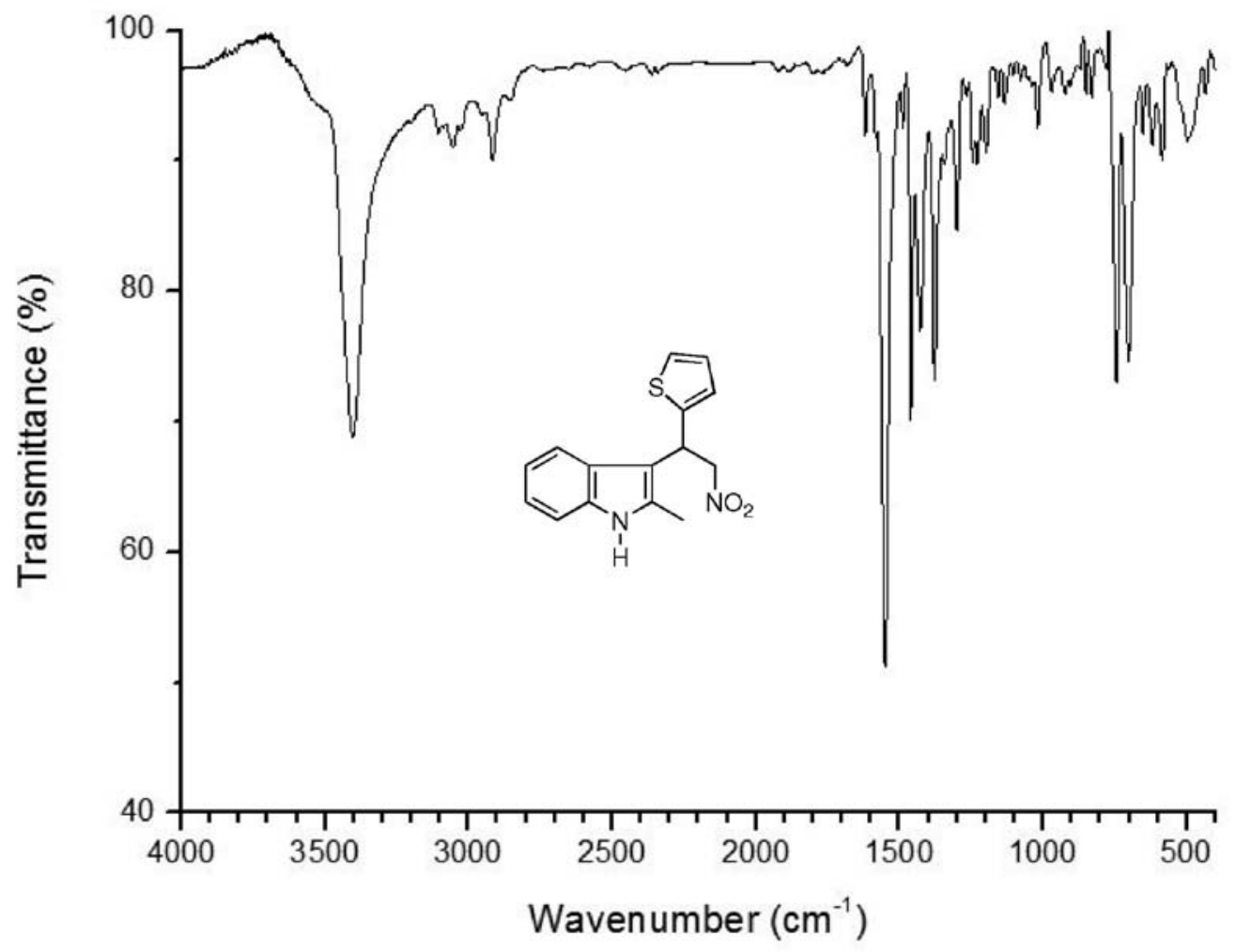

Figure S50. IR (KBr) of compound 5q.

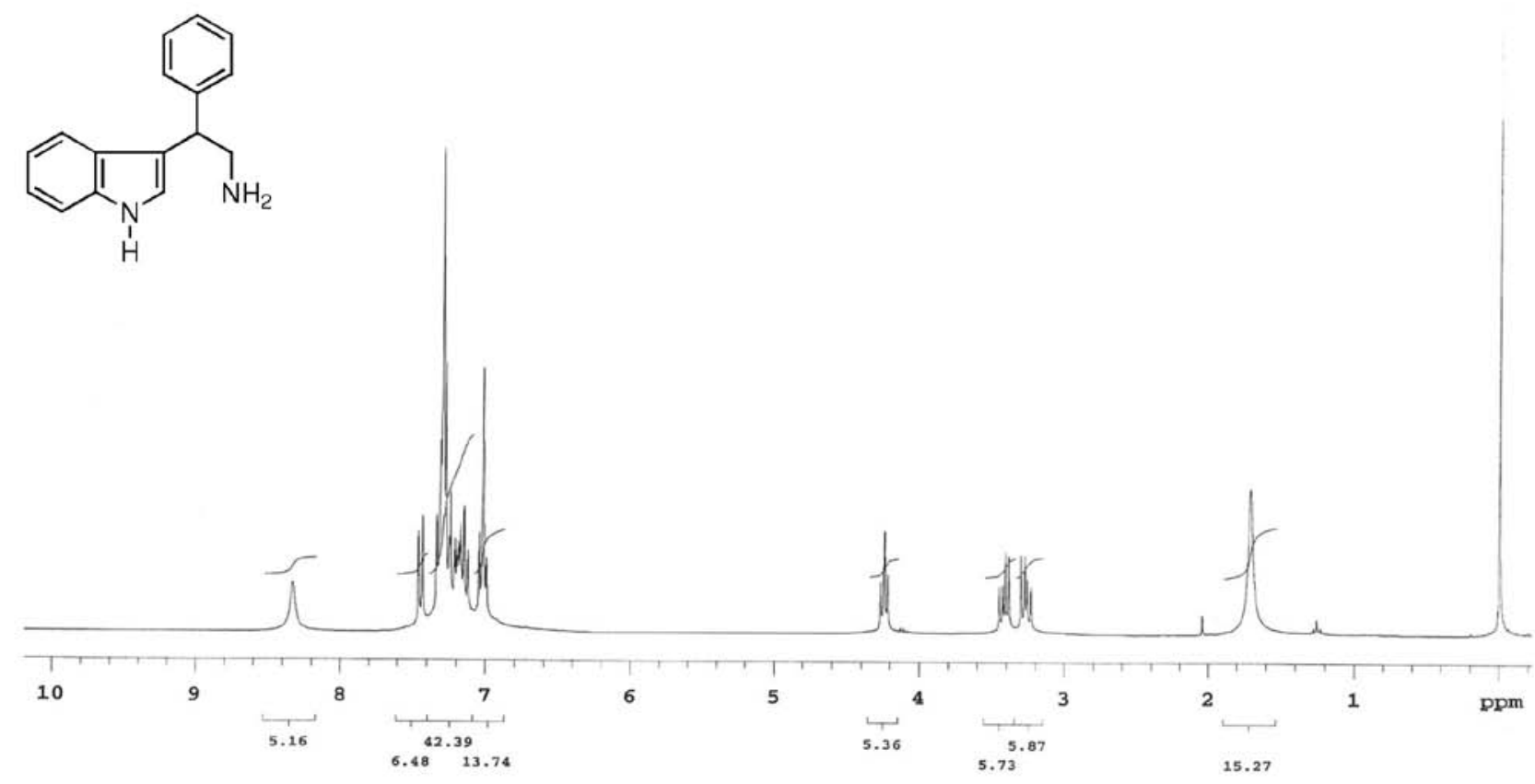

Figure S51. ${ }^{1} \mathrm{H} \mathrm{NMR}\left(\mathrm{CDCl}_{3}\right)$ of compound $\mathbf{8}$. 


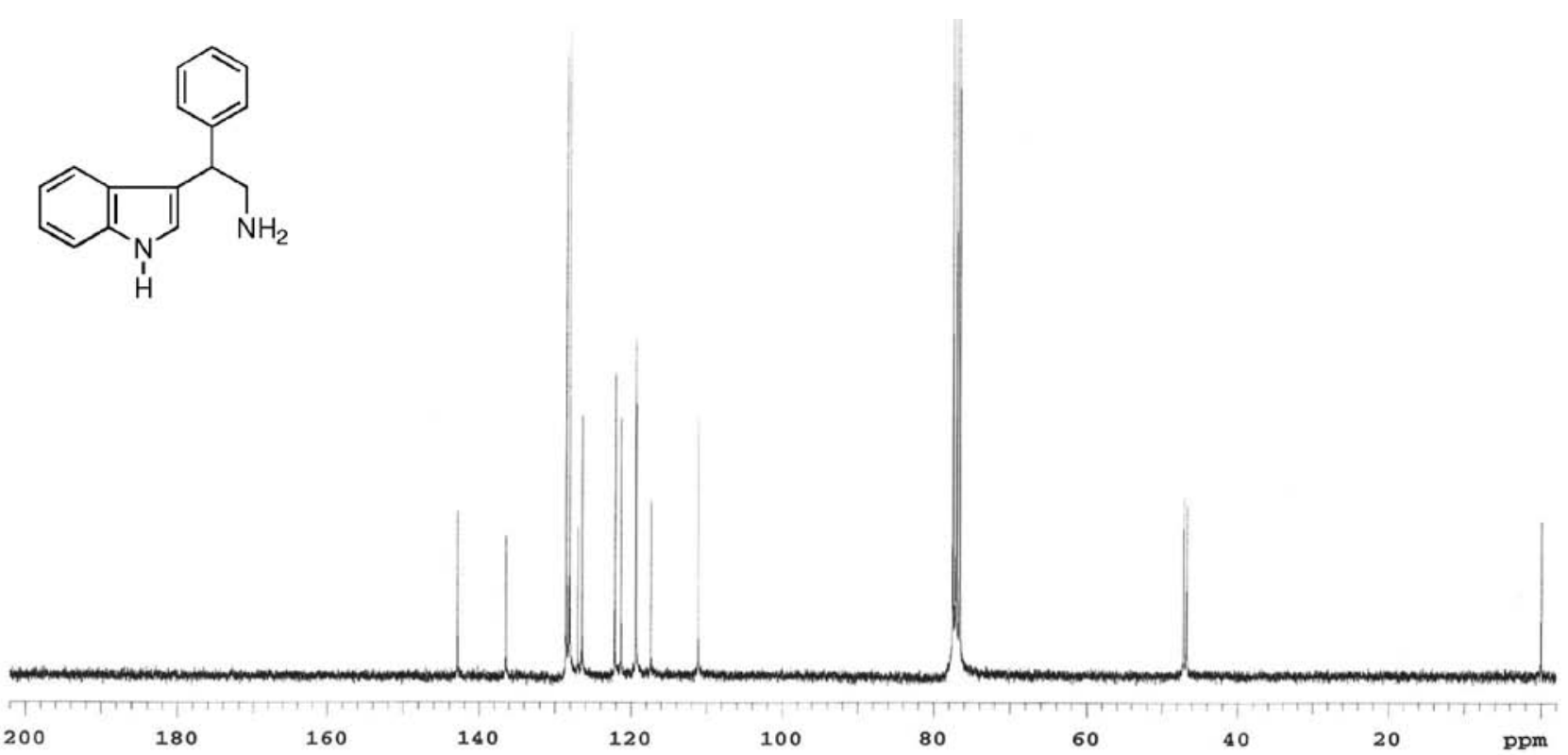

Figure S52. ${ }^{13} \mathrm{C} \mathrm{NMR}\left(\mathrm{CDCl}_{3}\right)$ of compound $\mathbf{8}$.

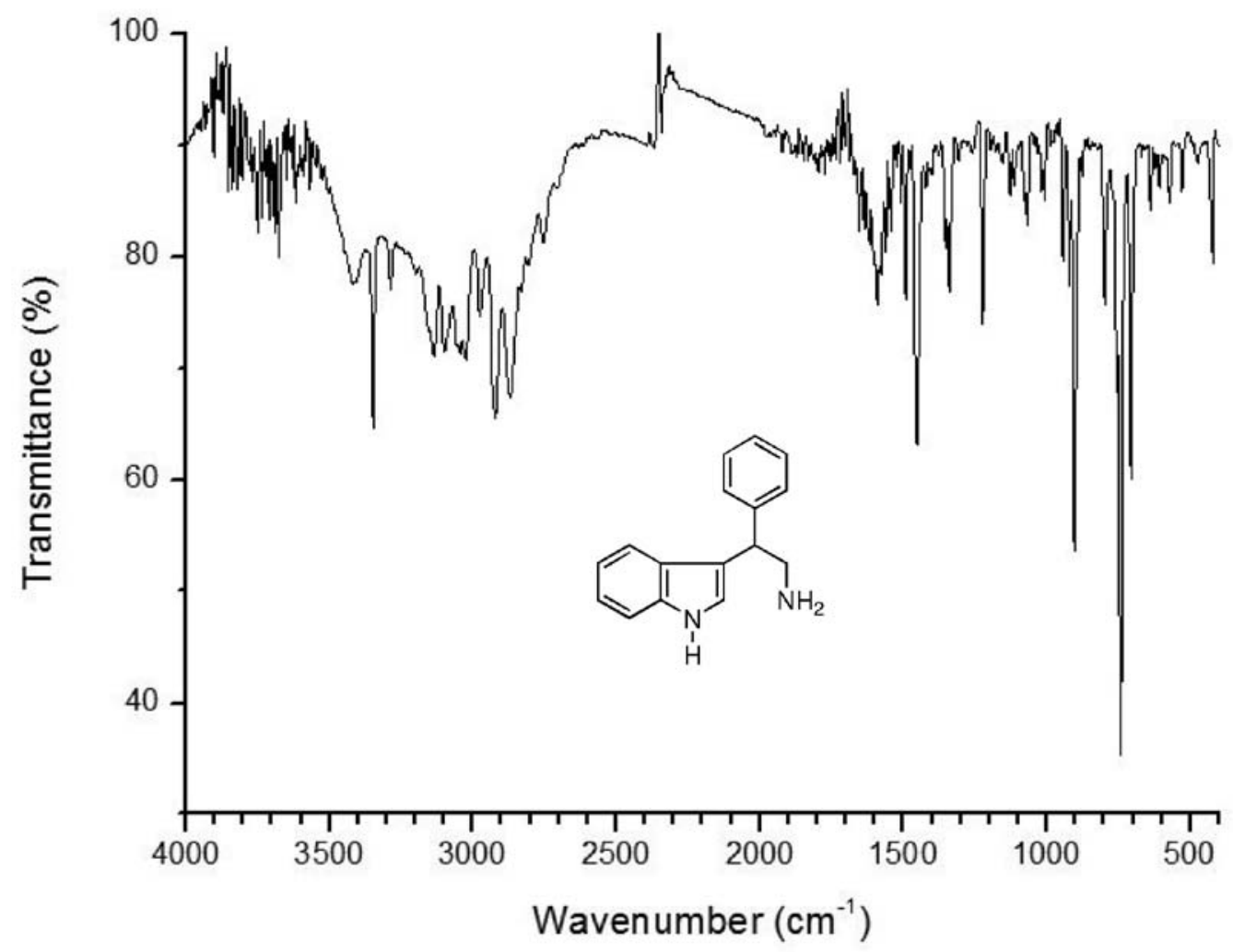

Figure S53. IR (KBr) of compound 8. 


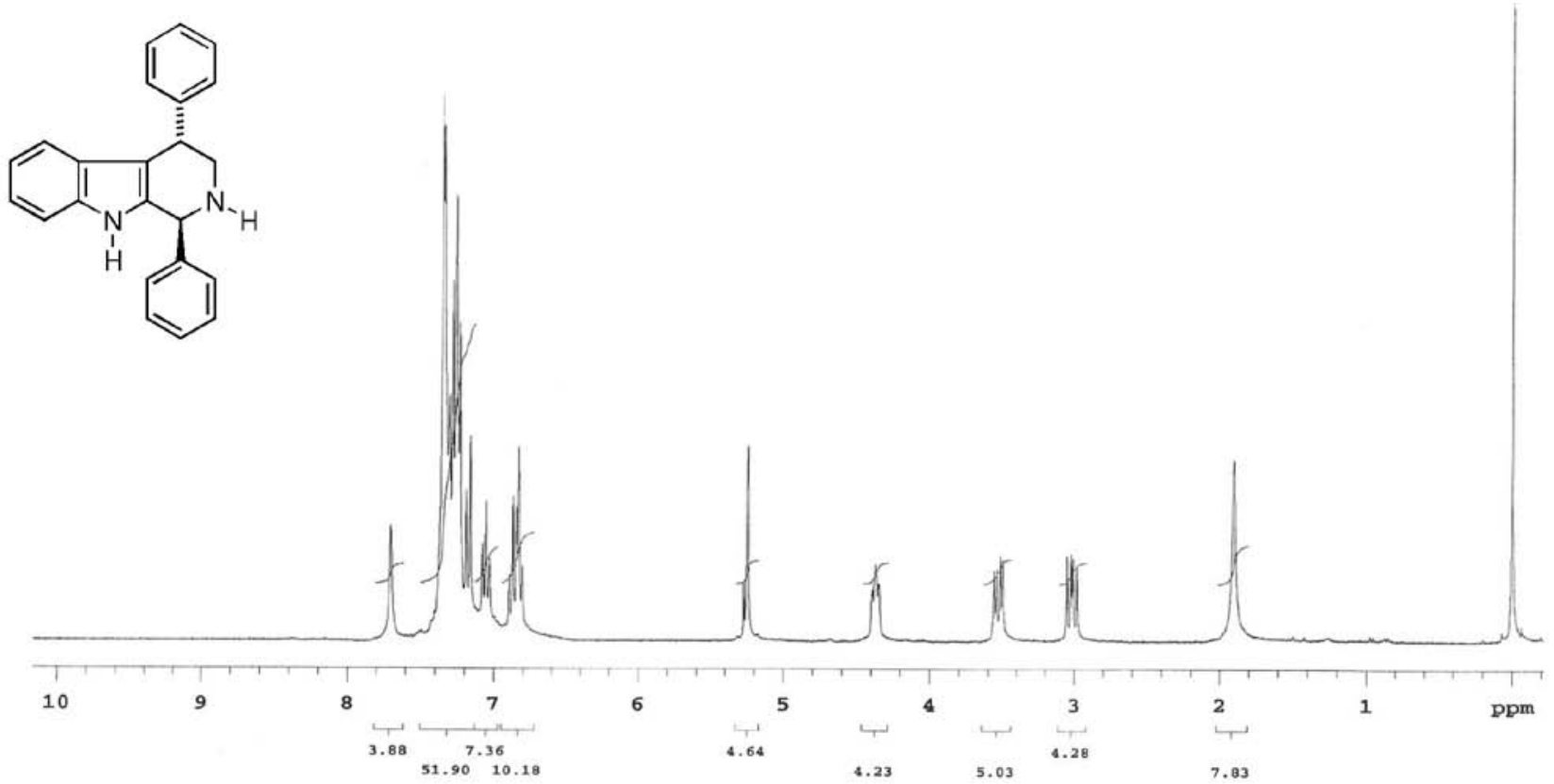

Figure S54. ${ }^{1} \mathrm{H} \mathrm{NMR}\left(\mathrm{CDCl}_{3}\right)$ of compound 9-trans.

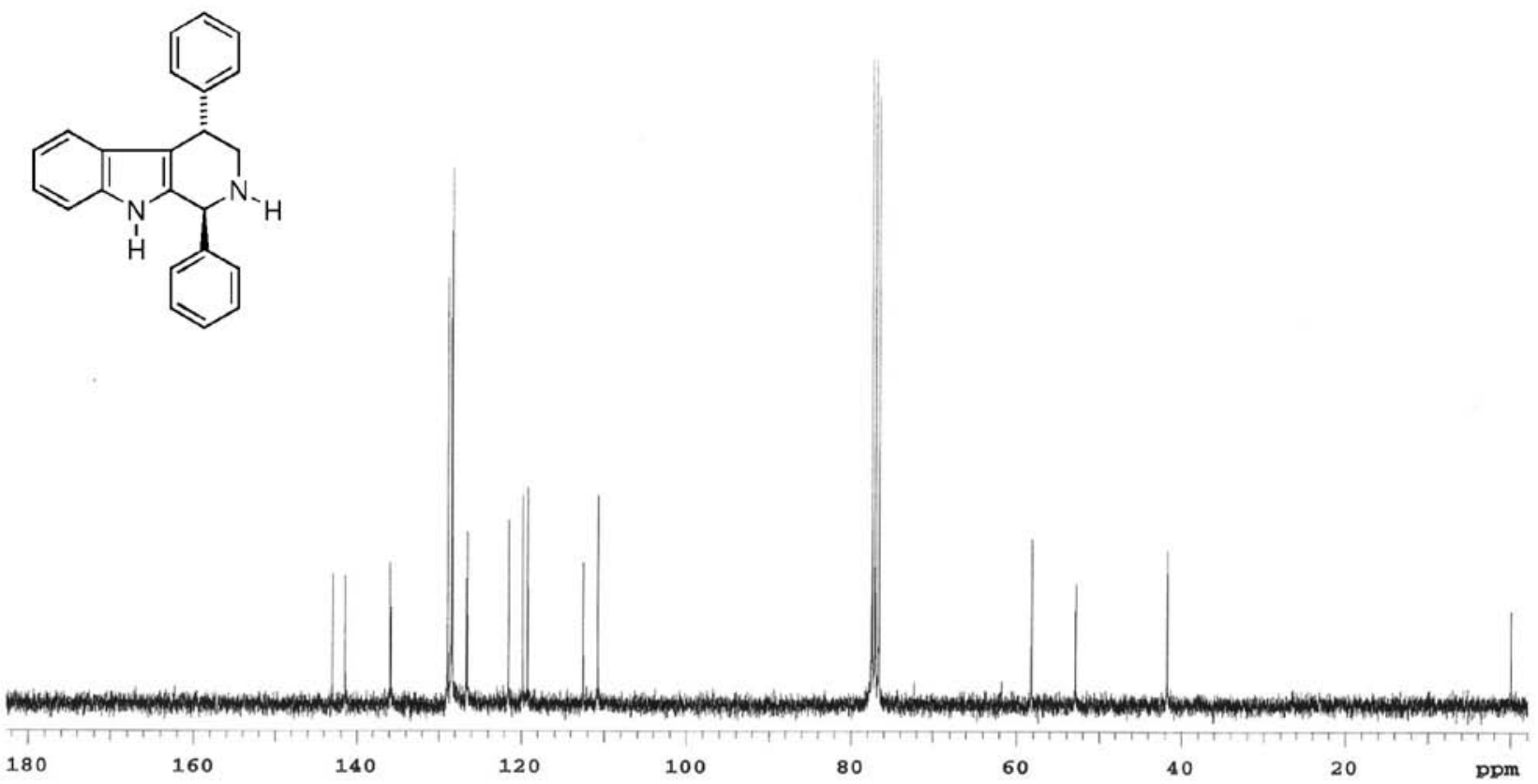

Figure S55. ${ }^{13} \mathrm{C}$ NMR $\left(\mathrm{CDCl}_{3}\right)$ of compound 9-trans. 


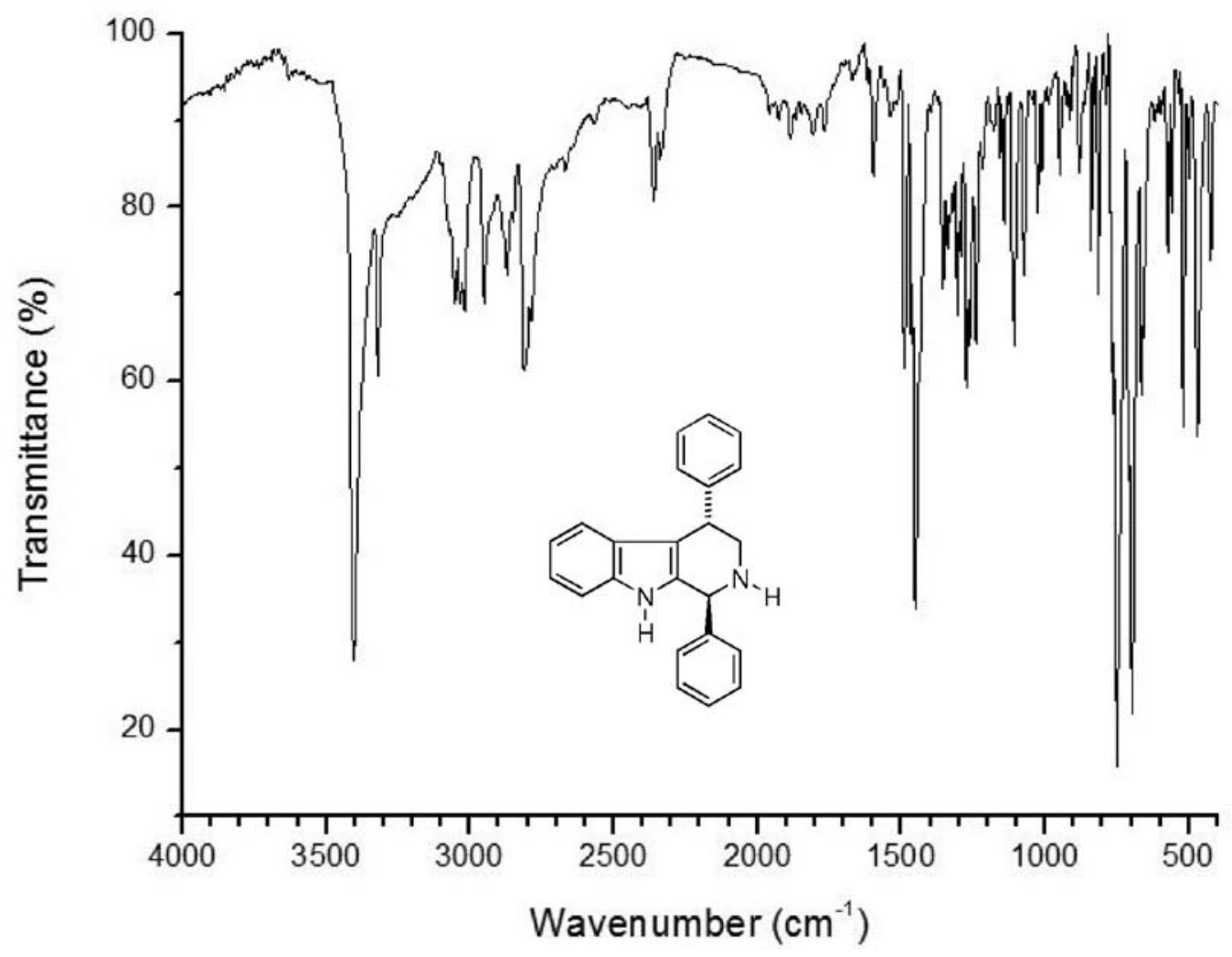

Figure S56. IR ( $\mathrm{KBr}$ ) of compound 9-trans.

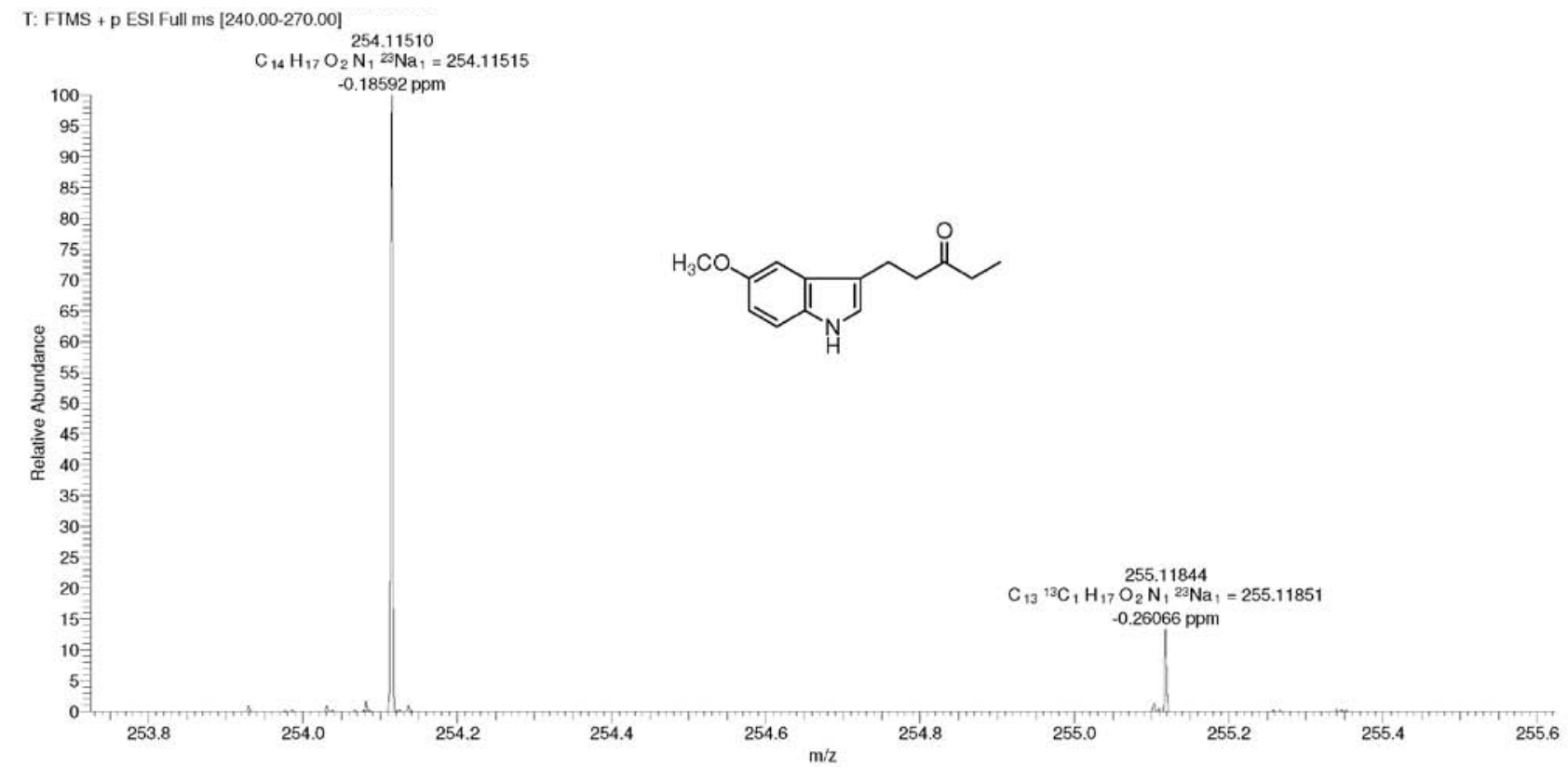

Figure S57. HRMS of compound 3g. 
S30

Metal Halide Hydrates as Lewis Acid Catalysts for the Conjugated Friedel-Crafts Reactions

J. Braz. Chem. Soc.

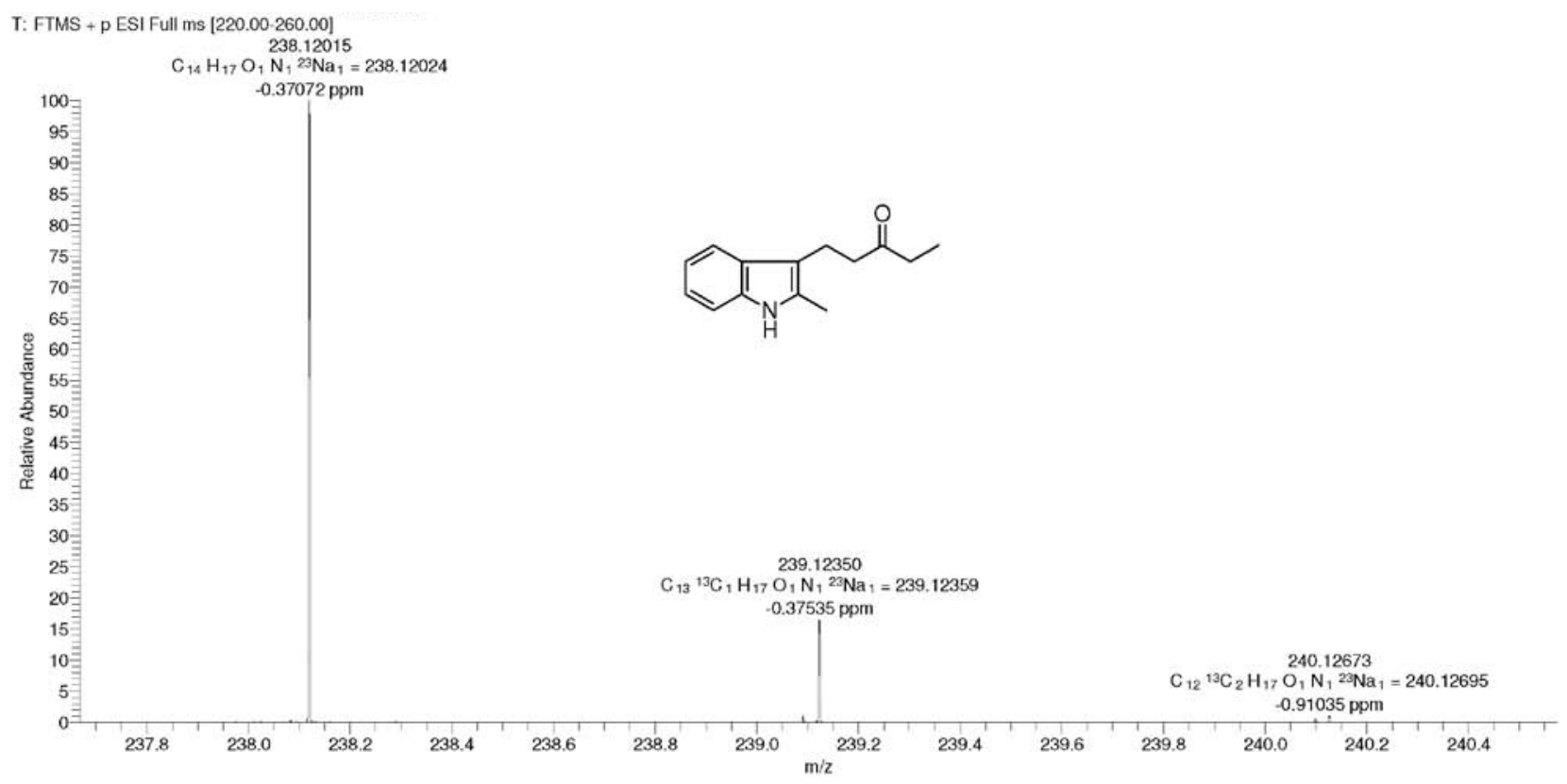

Figure S58. HRMS of compound 31 .

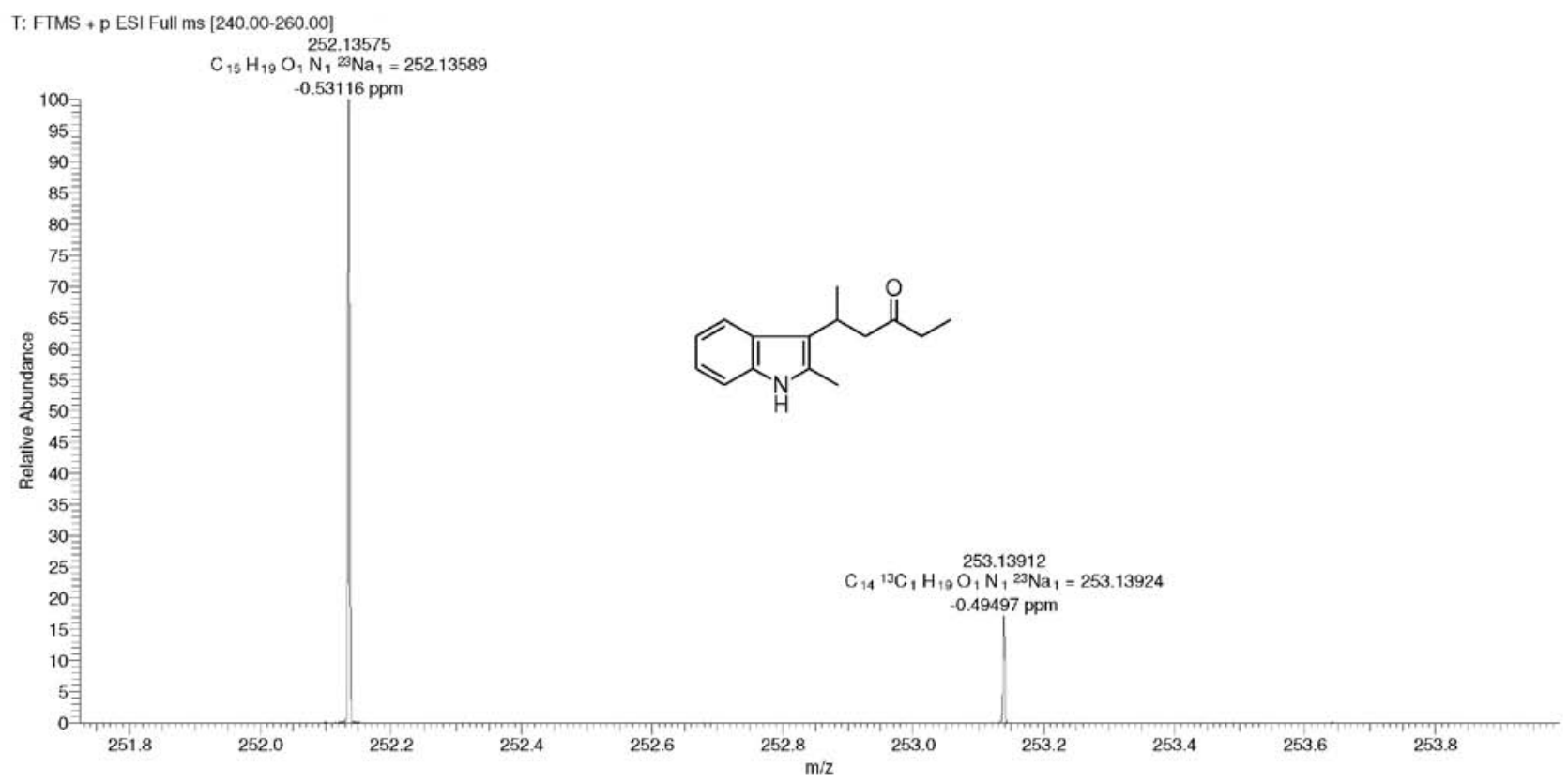

Figure S59. HRMS of compound Bk. 
Vol. 22, No. 4, 2011

Schwalm et al.

S31

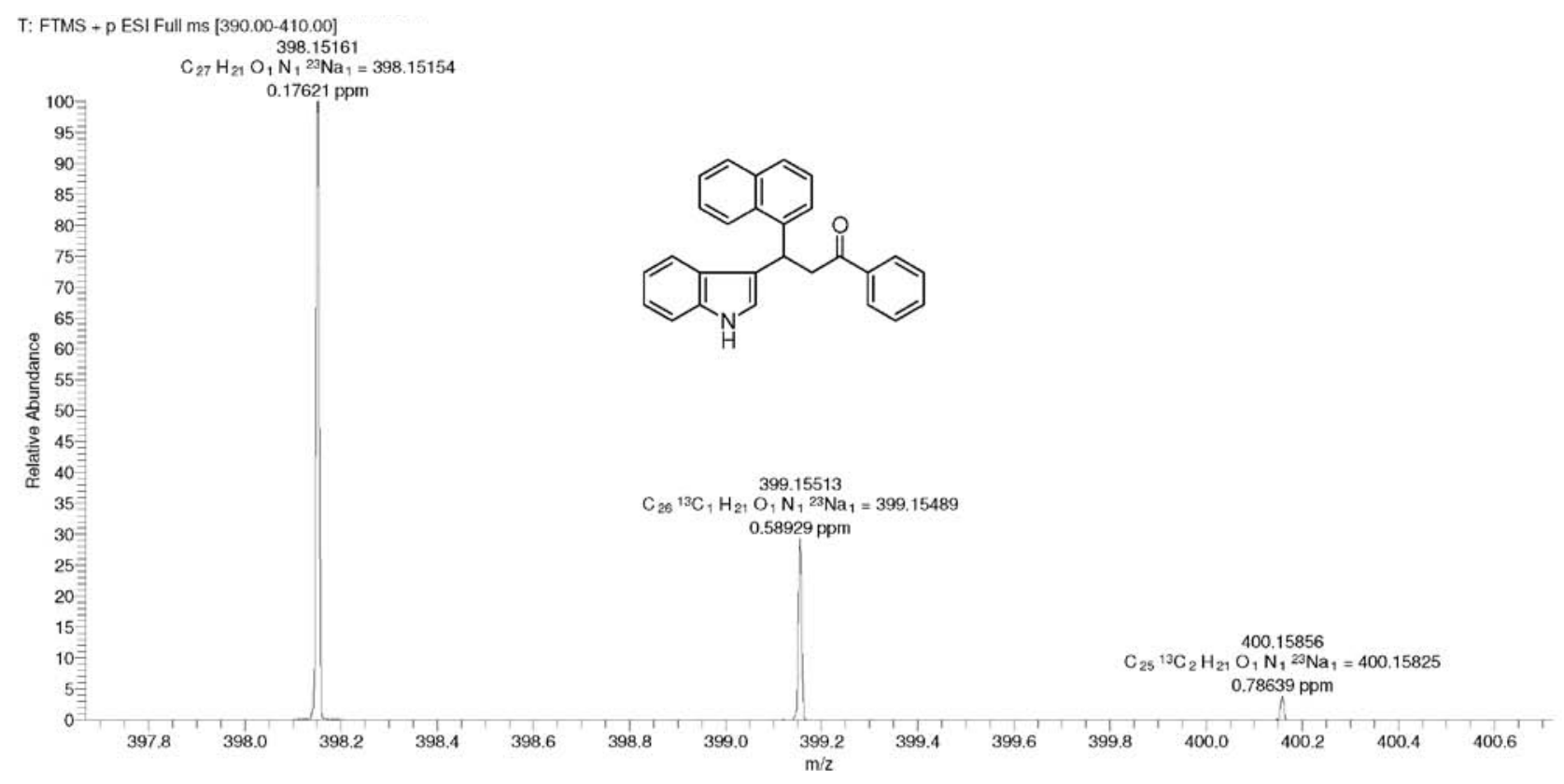

Figure S60. HRMS of compound 3s.

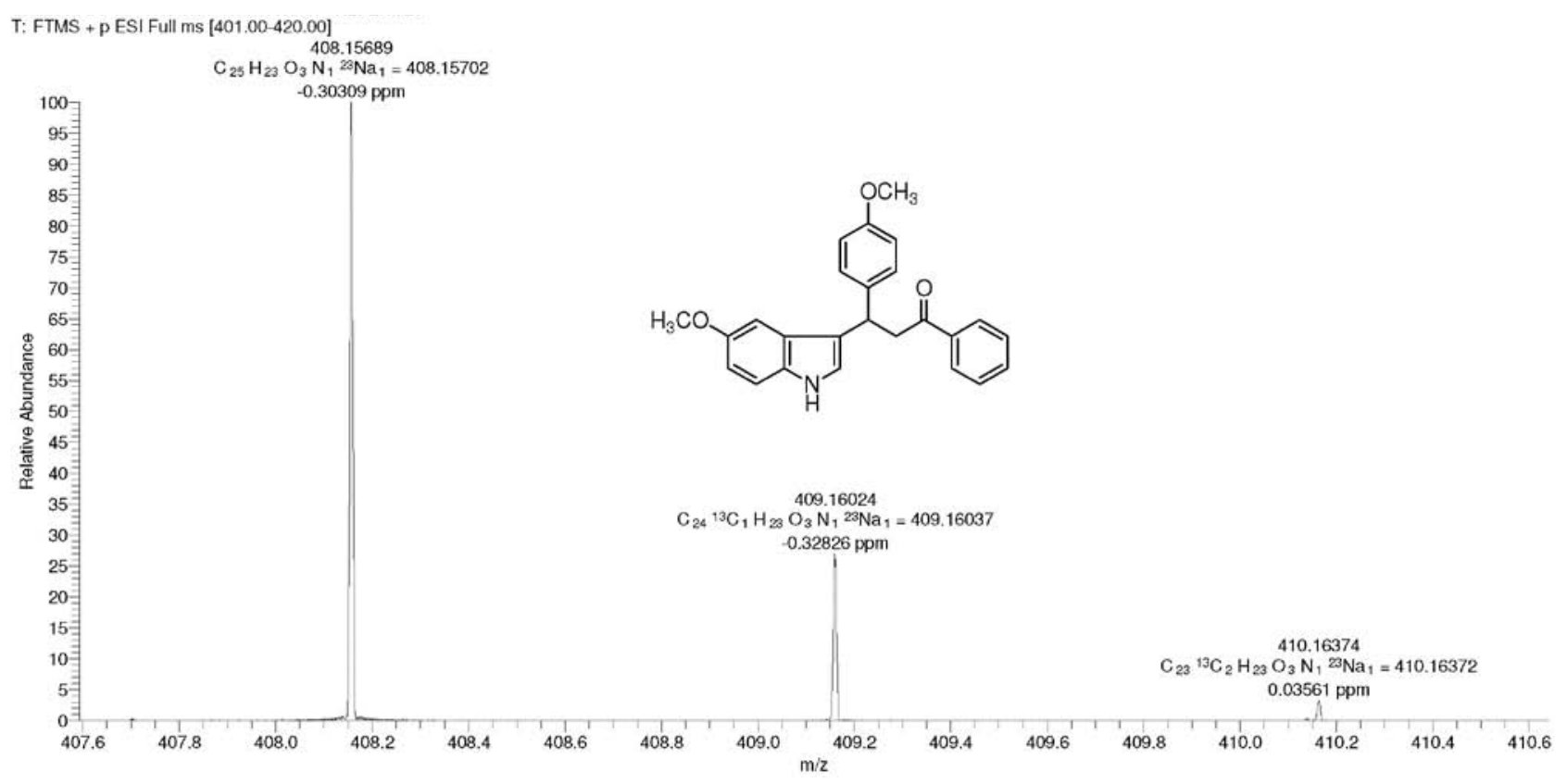

Figure S61. HRMS of compound 3x. 
S32

Metal Halide Hydrates as Lewis Acid Catalysts for the Conjugated Friedel-Crafts Reactions

J. Braz. Chem. Soc.

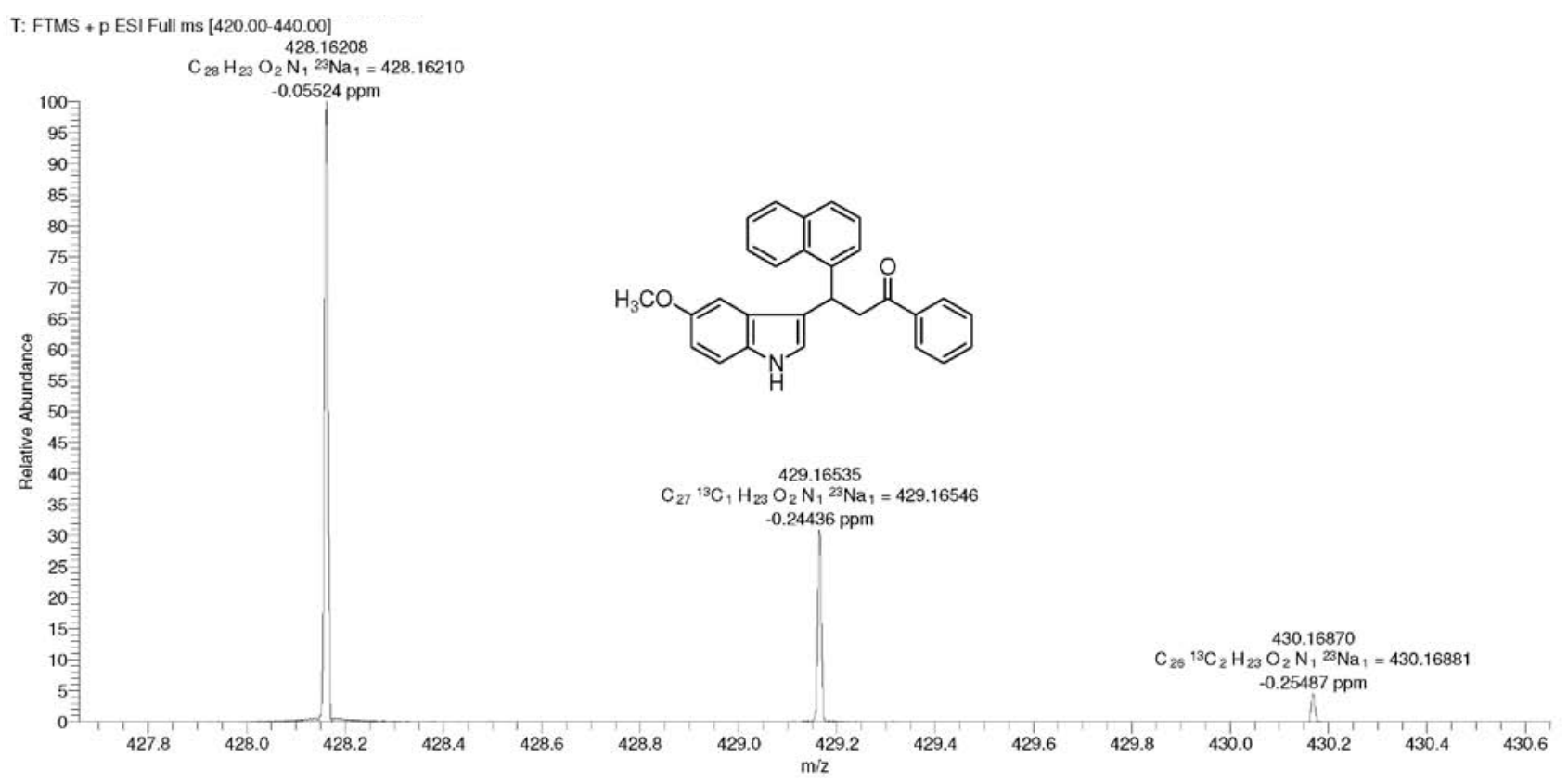

Figure S62. HRMS of compound Mw.

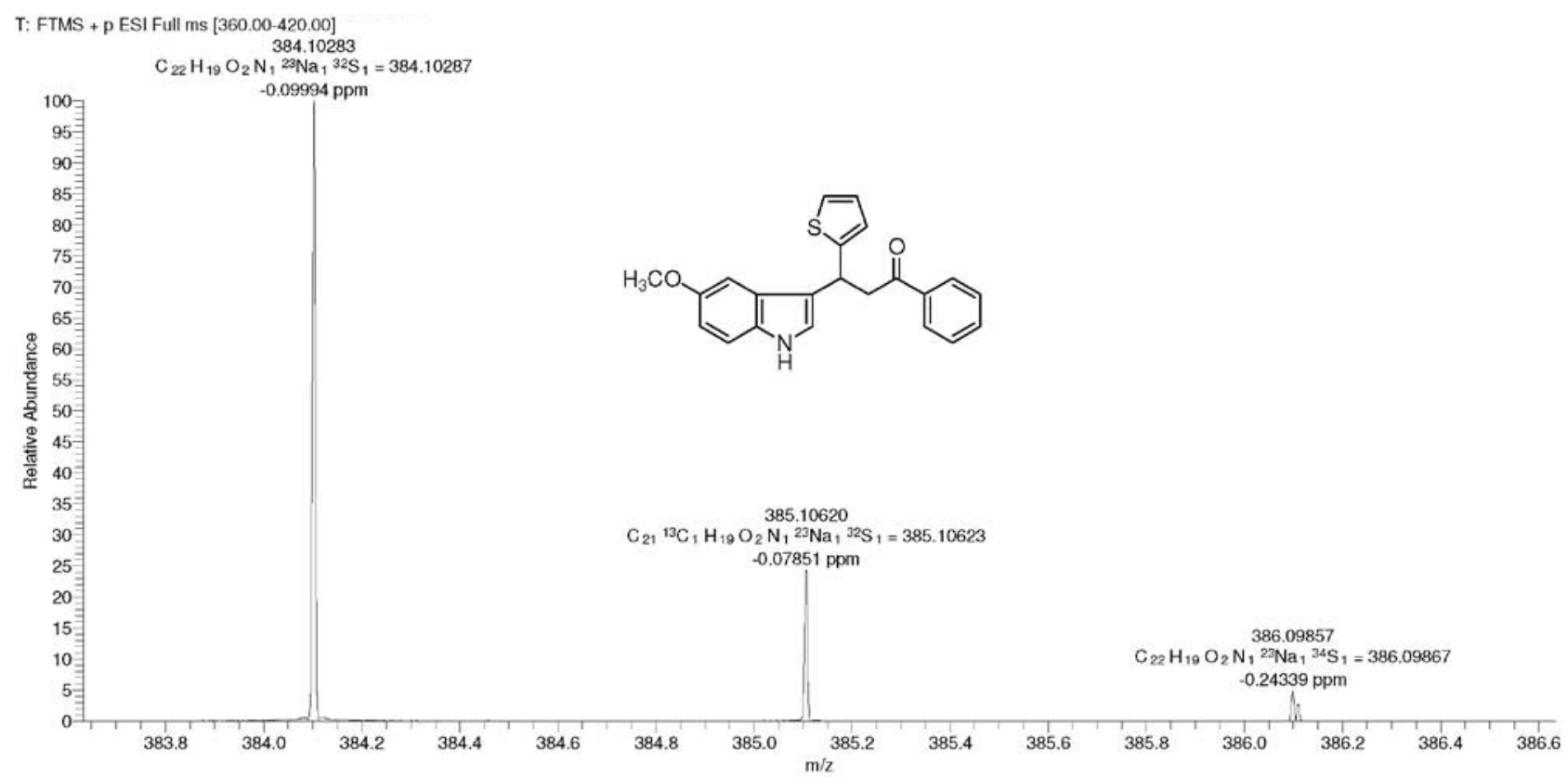

Figure S63. HRMS of compound By. 
T: FTMS + p ESI Full ms [320.00-360.00]
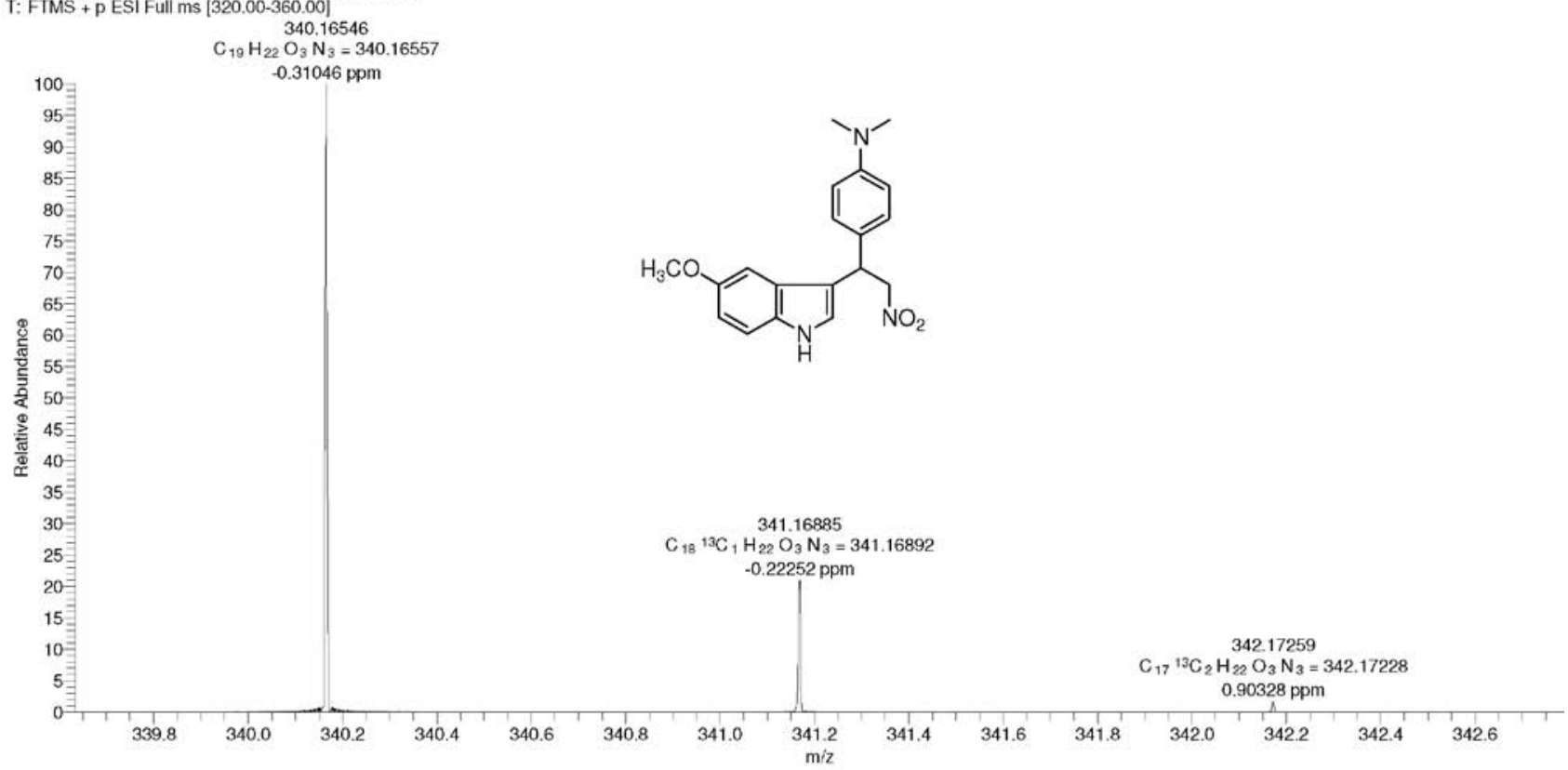

Figure S64. HRMS of compound $\mathbf{5 k}$.
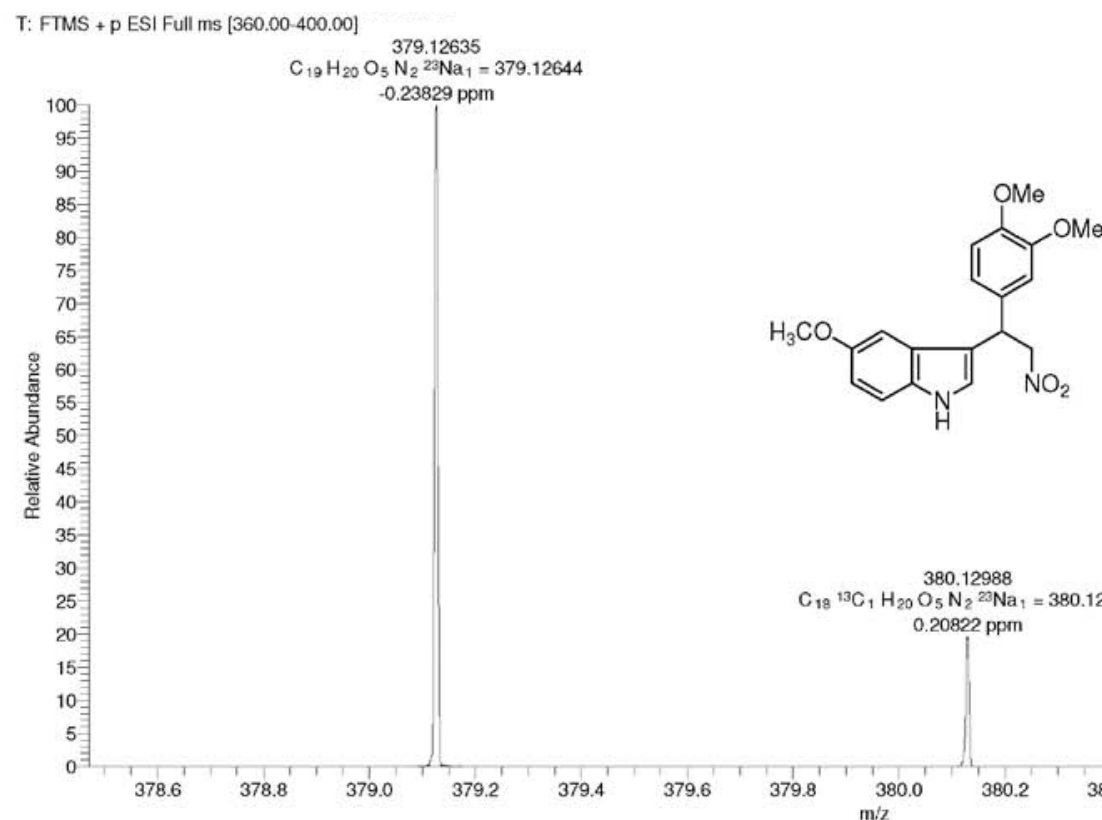

Figure S65. HRMS of compound 5 l. 
T: FTMS + p ESI Full ms [310.00-350.00]

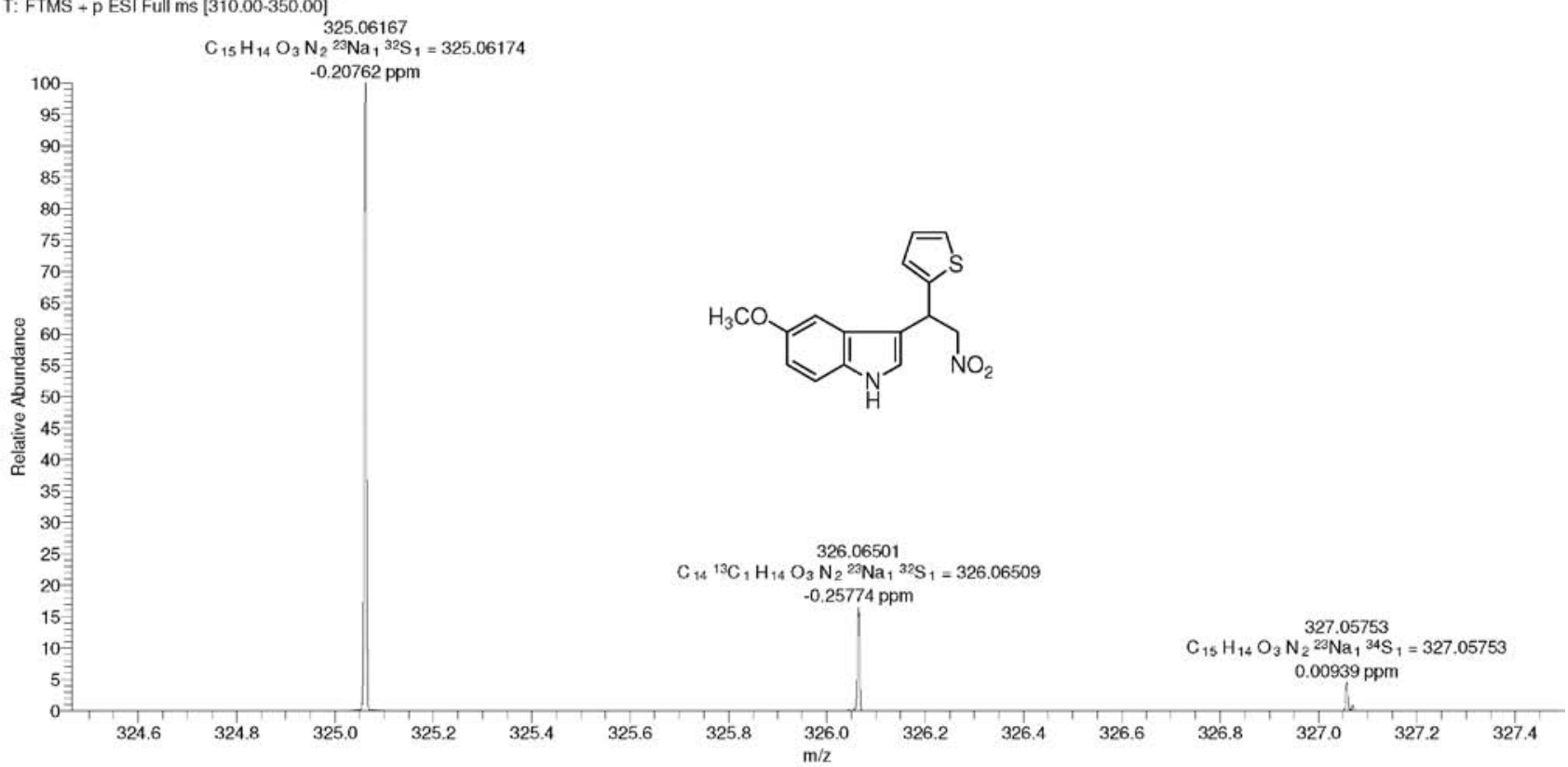

Figure S66. HRMS of compound 5 n.

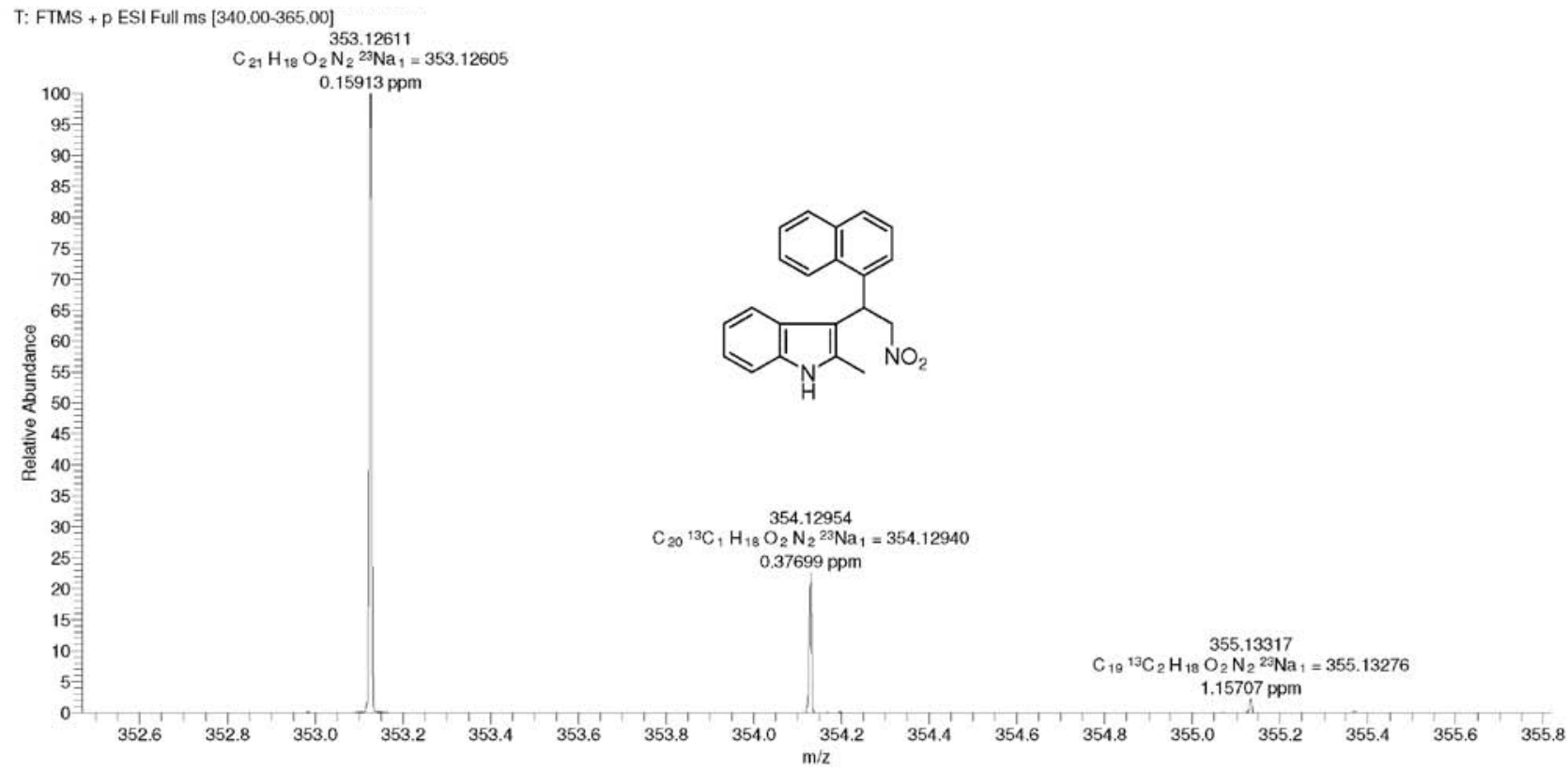

Figure S67. HRMS of compound 5p. 
T: FTMS + p ESI Full ms [300.00-320.00]

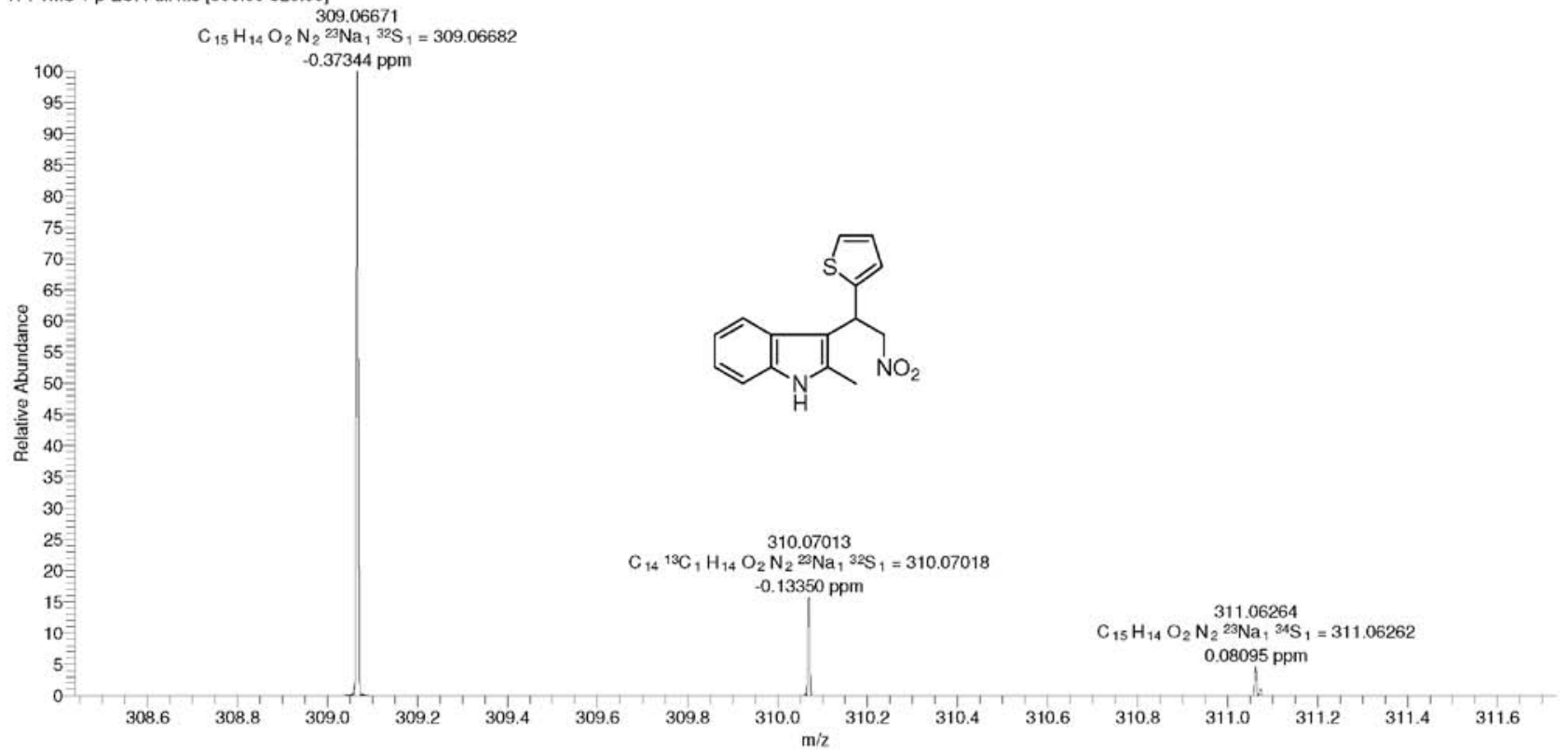

Figure S68. HRMS of compound 5q. 